\title{
High Temperature Fuel Cladding Chemical Interactions Between Unirradiated TRIGA Fuels and 304 Stainless Steel
}

Emmanuel Perez

Dennis D. Keiser, Jr. Bryan Forsmann

Dawn E. Janney

Jody Henley

Eric C. Woolstenhulme

February 2016

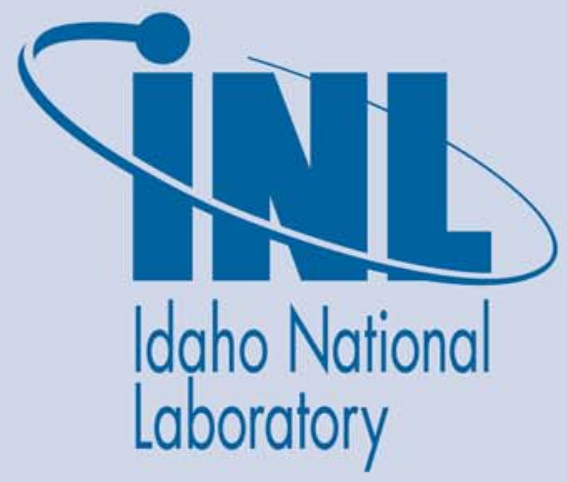

The INL is a U.S. Department of Energy National Laboratory operated by Battelle Energy Alliance 


\section{DISCLAIMER}

This information was prepared as an account of work sponsored by an agency of the U.S. Government. Neither the U.S. Government nor any agency thereof, nor any of their employees, makes any warranty, expressed or implied, or assumes any legal liability or responsibility for the accuracy, completeness, or usefulness, of any information, apparatus, product, or process disclosed, or represents that its use would not infringe privately owned rights. References herein to any specific commercial product, process, or service by trade name, trade mark, manufacturer, or otherwise, does not necessarily constitute or imply its endorsement, recommendation, or favoring by the U.S. Government or any agency thereof. The views and opinions of authors expressed herein do not necessarily state or reflect those of the U.S. Government or any agency thereof. 
INL/EXT-15-36708

Revision 0

\title{
High Temperature Fuel Cladding Chemical Interactions Between TRIGA Fuels and 304 Stainless Steel
}

\author{
Emmanuel Perez \\ Dennis D. Keiser, Jr. \\ Bryan Forsmann (Boise State University) \\ Dawn E. Janney \\ Jody Henley \\ Eric C. Woolstenhulme
}

February 2016

Idaho National Laboratory

Department of Homeland Security

Idaho Falls, Idaho 83415

http://www.inl.gov

Prepared for the

U.S. Department of Energy

Office of Materials Management and Minimization

Under DOE Idaho Operations Office

Contract DE-AC07-05ID14517 



\title{
High-Temperature Fuel Cladding Chemical Interactions between TRIGA Fuels and 304 Stainless Steel
}

\author{
INL/EXT-15-36708 \\ Revision 0
}

February 2016

\section{Approved by:}
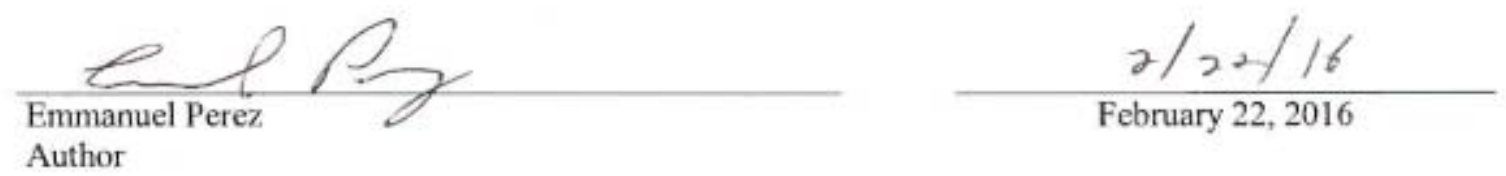

Dis Conktaknine for Keiser por Author

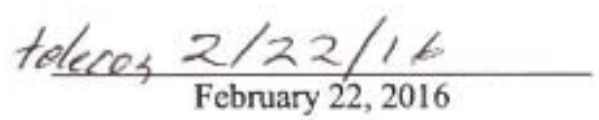

$\sum n_{2}$

Eric C. Woolstenhulme

Manager/Contributor 



\begin{abstract}
High-temperature fuel cladding chemical interactions (FCCI) between Training, Research, Isotopes, General Atomics (TRIGA) fuel elements and the 304 stainless steel (304SS) are of interest to develop an understanding of the fuel behavior during transient reactor scenarios, such as a loss-of-coolant-accident (LOCA). TRIGA fuels are composed of uranium (U) particles dispersed in a zirconium-hydride $(\mathrm{Zr}-\mathrm{H})$ matrix. In the reactor, the fuel is encased in 304SS or Incoloy 800 clad tubes. At high temperatures, the fuel has the potential to interact with the cladding, resulting in FCCI.

A number of FCCI can take place in this system. Interactions can be expected between the cladding and the $\mathrm{Zr}-\mathrm{H}$ matrix, and/or between the cladding and the U-particles. Other interactions may be expected between the $\mathrm{Zr}-\mathrm{H}$ matrix and the U-particles. Furthermore, the fuel contains erbium-oxide $\left(\mathrm{Er}_{2} \mathrm{O}_{3}\right)$ additions. Interactions can also be expected between the $\mathrm{Er}_{2} \mathrm{O}_{3}$, the cladding, the $\mathrm{Zr}-\mathrm{H}$ and the U-particles. The overall result is that very complex interactions may take place as a result of fuel and cladding exposures to high temperatures. These interactions must be understood to ensure safe operation of the fuels under normal and transient conditions. This report discusses the characterization of the baseline fuel microstructure in the as-received state (prior to exposure to high temperature), characterization of the fuel after annealing at $950^{\circ} \mathrm{C}$ for 5,16 , and 24 hours, and the results from diffusion couple experiments carried out at $1000^{\circ} \mathrm{C}$ for 5 and 24 hours. Emphasis is given to the interaction regions between TRIGA fuels in contact with 304SS and the development of low-melting-temperature phases. Characterization was carried out via scanning electron microscopy (SEM) and transmission electron microscopy (TEM) with sample preparation via focused ion beam in situ lift-out technique.
\end{abstract}




\section{CONTENTS}

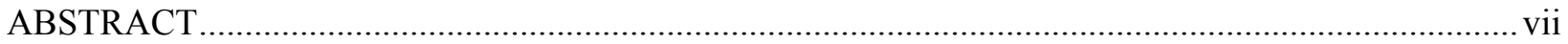

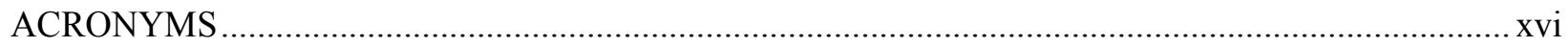

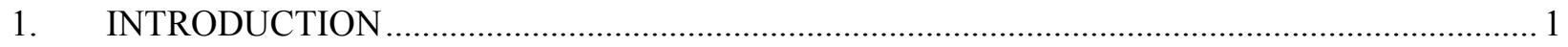

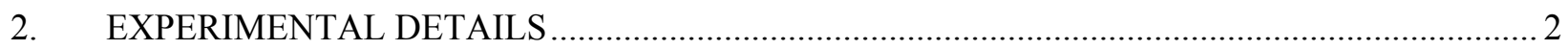

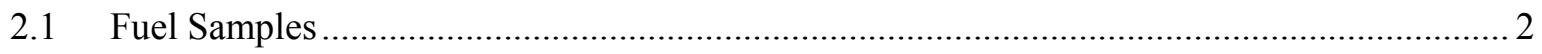

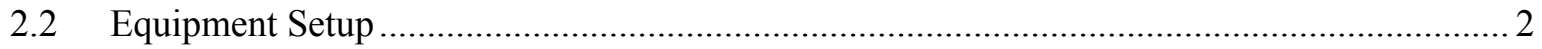

2.3 Diffusion Couples and Fuel Annealing Experiments........................................................ 6

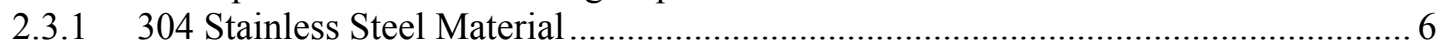

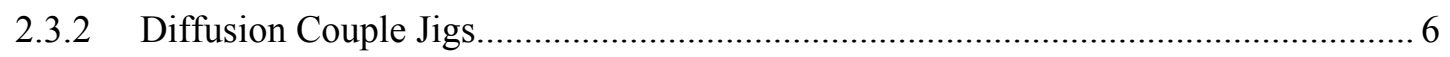

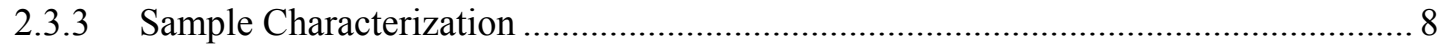

3. RESULTS

3.1 Characterization of Baseline (As-Received) TRIGA Fuel................................................. 9

3.1.1 Characterization by Scanning Electron Microscopy ............................................... 9

3.1.2 Characterization by Transmission Electron Microscopy ....................................... 12

3.1.3 Analyses of Fine Features and Extra Reflections in Selected Diffraction

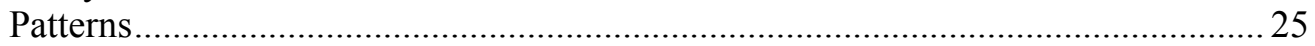

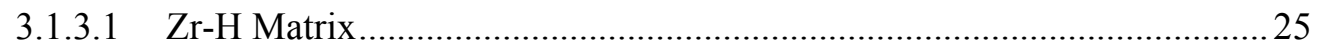

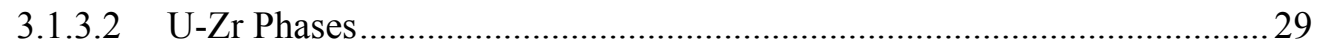

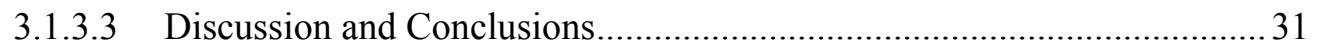

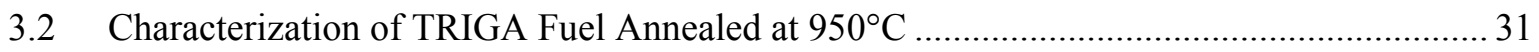

3.2.1 Characterization by Scanning Electron Microscopy ............................................... 33

3.2.2 Characterization by Transmission Electron Microscopy …..................................... 41

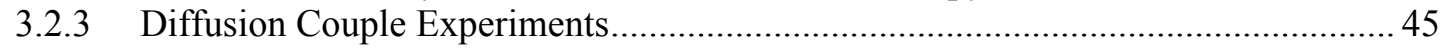

3.2.4 Characterization by Scanning Electron Microscopy ............................................... 46

3.2.5 Characterization by Transmission Electron Microscopy …...................................... 57

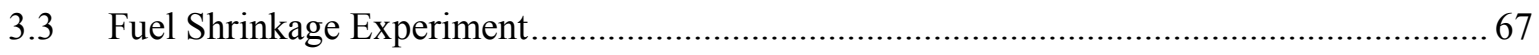

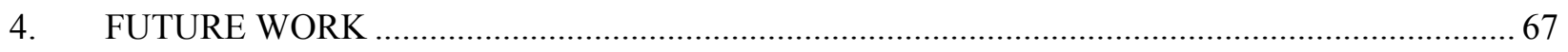

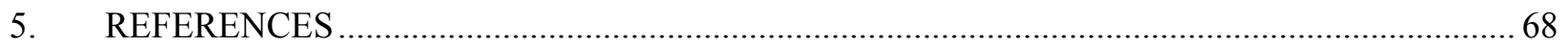




\section{FIGURES}

Figure 1. (a) TRIGA fuel disks used in this study; (b) fuel samples cut from a randomly selected fuel disk. 2

Figure 2. Box furnace utilized to carry out annealing and diffusion couple experiments. 3

Figure 3. Schematic of the furnace setup inside of the glovebox including an Ar-gas tank external to the glovebox with an inline gas purifier...

Figure 4. (a) and (b) The setup of the furnace inside of the glovebox from two different angles showing the Ar-gas feed line with a flowmeter, two thermocouples inserted through a port on furnace door, and two thermocouples placed outside of the furnace. (c) The Ar-gas line setup with a gas purifier inline. (d) A more-detailed picture of the gas purifier. (e) A picture of the Ar-gas tank with the details of the gas..

Figure 5. Four jig designs used to perform diffusion couple experiments. The jig designs are arranged from original to most recent design, $(a \rightarrow d)$.

Figure 6. Schematic of a diffusion couple assembly onto a fourth-generation jig with top and side view perspectives. 8

Figure 7. Backscatter electron micrographs showing the typical microstructure of fresh TRIGA fuel analyzed in this study. (a) Emphasizes the general microstructure and apparent grain boundaries. (b) Typical dark contrast grains found throughout the microstructure.

Figure 8. BE micrographs showing (a) the fuel microstructure with a grain boundary included, and (b) a detailed section of a grain boundary containing $U$ and another distinct phase (marked by white arrows).

Figure 9. Energy dispersive spectroscopy patterns of the (a) matrix, (b) U-particles, (c) dark contrast grains, and (d) the phase observed within the grain boundaries.

Figure 10. X-ray maps of a selected region of the TRIGA fuel showing (a) a secondary electron micrograph of selected region of TRIGA fuel for x-ray mapping; x-ray mapping of (b) $\mathrm{Zr}$, (c) U, (d) Er, and (e) O intensities. (Note: The oxygen intensity was enhanced to emphasize oxygen concentration in the grain boundaries.)

Figure 11. HAADF micrographs of five TEM samples prepared to capture the different microstructural features found in the fresh TRIGA fuel: (a) general microstructure, (b) and (c) grain boundaries with Er-grains, and (d) and (e) Zr-rich phase (dark contrast phase in Figure $7[\mathrm{~b}])$.

Figure 12. (a) and (b) Typical high-angle annular dark field micrographs of U-particles in the $\mathrm{Zr}-\mathrm{H}$ matrix displaying likely interaction regions with the corresponding EDS patterns of the (c) Zr-H matrix, (d) unreacted U-particles, and (e) C-U-Zr interaction region between $\mathrm{U}$ and $\mathrm{Zr}-\mathrm{H}$..

Figure 13. (a) and (b) HAADF micrographs of typical $\mathrm{U}$ and Er-O particles in the Zr-H matrix showing displaying likely Er-O-Zr and Er-O-U interaction regions with the corresponding EDS patterns of the (c) Er-O, (d) Er-O-U, and (e) Er-O-Zr interaction regions.

Figure 14. HAADF micrographs (a) through (d) at increasing magnifications of the secondary Zr-rich particles observed in fuel matrix microstructure. 
Figure 15. BF micrographs (a) and (b) at increasing magnification of the secondary Zr-rich particles observed in fuel matrix microstructure emphasizing microstructural features.

Figure 16. (a) HAADF micrograph of a selected interaction region between a U-particle and the $\mathrm{Zr}-\mathrm{H}$ matrix. (b) The concentration profile collected at the red line, and between the dashed lines, shown in (a).

Figure 17. (a) HAADF micrograph of a selected interaction region between an Er grain and the Zr-H matrix. (b) The concentration profile collected at the red line, and between the dashed lines, shown in (a).

Figure 18. (a) HAADF micrograph of a selected interaction region between a U-particle and an Er grain; (b) The concentration profile collected at the red line, and between the dashed lines, shown in (a).

Figure 19. (a) A BF micrograph of a selected grain (marked by a red arrow) from the $\mathrm{Zr}-\mathrm{H}$ matrix. (b), (c), and (d) are the corresponding electron diffraction spot patterns, indexed as the tetragonal- $\mathrm{H}_{2} \mathrm{Zr}$ phase, displaying three different zone axes from the selected grain.......

Figure 20. (a) A BF micrograph of a selected grain (marked by a red arrow) from a U-particle.

(b), (c), and (d) show the corresponding diffraction patterns, indexed as the orthorhombic $\alpha-U$ phase, displaying three different zone axes from the selected grain.

Figure 21. (a) A BF micrograph of a selected grain (marked by a red arrow) from an Er-O. (b), (c), and (d) show the corresponding diffraction patterns, indexed as the cubic- $\mathrm{Er}_{2} \mathrm{O}_{3}$ phase, displaying three different zone axes from the selected grain.

Figure 22. (a) Bright field micrograph of a selected from a lamella within a region similar to that shown in Figure 15. (b), (c), and (d) show the corresponding unindexed diffraction spot patterns displaying three different zone axes from the selected grain.

Figure 23. (a) Bright field micrograph of a selected U-particle/Zr-H-matrix interaction region. (b), (c), and (d) show the corresponding unindexed diffraction spot patterns displaying three different zone axes from the selected grain.

Figure 24. Selected Zr-H grain (grain 1, zone 1) in the matrix. (a) Bright field micrograph of the selected area with solid and dashed lines showing orientations of two sets of linear features. (b) Central part of diffraction pattern. Arrows show streaks whose orientation is consistent with diffraction from features parallel to the solid lines in the image. 26

Figure 25. Selected Zr-H grain (grain 1, zone 2) in the matrix. (a) Bright field micrograph of the selected area where solid and dashed lines show orientations of two sets of linear features. (b) Central part of diffraction pattern. Small arrows show streaks whose orientation is consistent with diffraction from features parallel to the solid lines in the image. Large arrows show examples of reflections in the centers of the streaks.

Figure 26. Selected Zr-H grain (Grain 1, zone 3) in the matrix. (a) Bright field micrograph of the selected area where solid and dashed lines show orientations of two sets of linear features. (b) Central part of diffraction pattern. Small arrow shows an example of a faint streak whose orientation is consistent with diffraction from features parallel to the solid lines in the image. Large arrow shows an example of a faint streak whose orientation is consistent with diffraction from features parallel to the dotted lines in the image. 
Figure 27. Selected Zr-H grain (Grain 2, zone 3) in the matrix. (a) Bright field micrograph of the selected area where white lines show orientations of linear features in different areas.

(b) Central part of diffraction pattern. 28

Figure 28. Selected Zr-H grain (Grain 3, Zone 1) in the matrix. (a) Bright field micrograph of the selected area with black line showing orientation of closely spaced linear features. (b) Corresponding diffraction pattern, with arrows showing example of faint paired reflections. 28

Figure 29. Selected Zr-H grain (Grain 3, zone 2) in the matrix. (a) Bright field micrograph of the selected area with black line showing orientation of closely spaced linear features. (b) Corresponding diffraction pattern, with arrows showing examples of very faint elongated reflections.

Figure 30. Selected U-Zr grain (Grain 3, Zone 2). (a) and (b) Bright field micrograph of the selected areas at different magnifications. Arrows in (a) show examples of dark spots in a regular pattern. (c) Diffraction pattern, with small arrows showing a line of faint reflections and large arrows showing streaks between reflections. (d) Diffraction pattern, with faint reflections (e.g., at small arrows) forming a pattern similar to bright reflections (large arrows) but offset by a consistent distance and direction... 30

Figure 31 . Temperature profiles generated from the TRIGA fuel annealing process at $950^{\circ} \mathrm{C}$ for (a) 5, (b) 16, and (c) 24 hours. 32

Figure 32. TRIGA fuel microstructure after annealing at $950^{\circ} \mathrm{C}$ for 5 hours: (a) low and (b) higher magnification BE micrographs 33

Figure 33. TRIGA fuel microstructure after annealing at $950^{\circ} \mathrm{C}$ for 16 hours: (a) low and (b) higher magnification $\mathrm{BE}$ micrographs. .33

Figure 34. TRIGA fuel microstructure after annealing at $950^{\circ} \mathrm{C}$ for 24 hours: (a) low and (b) higher magnification BE micrographs. .34

Figure 35. (a) A selected area of the TRIGA fuel annealed at $950^{\circ} \mathrm{C}$ for 5 hours. (b) -(d) Qualitative x-ray maps showing the presence and location of (b) Zr, (c) U, and (d) Er in the fuel microstructure.

Figure 36. (a) A selected area of the TRIGA fuel annealed at $950^{\circ} \mathrm{C}$ for 16 hours. (b)-(d) Qualitative x-ray maps showing the presence and location of (b) Zr, (c) U, and (d) Er in the fuel microstructure.

Figure 37. (a) A selected area of the TRIGA fuel annealed at $950{ }^{\circ} \mathrm{C}$ for 5 hours. (b)-(d) Qualitative x-ray maps showing the presence and location of (b) $\mathrm{Zr}$, (c) U, and (d) Er in the fuel microstructure.

Figure 38. Typical EDS patterns for (a) matrix, (b) dark-gray phase, and (c) mid-gray phase regions in fuel sample annealed at $950^{\circ} \mathrm{C}$ for 5 hours. .38

Figure 39. Typical EDS patterns for (a) matrix, (b) dark-gray phase, (c) mid-gray phase, and (d) acicular regions in fuel sample annealed at $950^{\circ} \mathrm{C}$ for 16 hours. 39

Figure 40. Typical EDS patterns for (a) matrix, (b) dark-gray phase, (c) mid-gray phase, and (d) acicular regions in fuel sample annealed at $950^{\circ} \mathrm{C}$ for 24 hours.

Figure 41. (a) BE micrograph illustrating the region selected (marked by an arrow) for characterization by TEM. (b) HAADF micrograph of the prepared TEM sample.

Figure 42. EDS patterns of the (a) matrix and the (b) secondary phase captured in the TEM sample in Figure 41(b). 
Figure 43. Example of diffraction patterns from Grain 1 depicting three distinct zone axes. Each zone axis closely matches the $\mathrm{H}_{1.6} \mathrm{Zr}$ and $\mathrm{H}_{2} \mathrm{Zr}$ phases. The $\mathrm{H}_{2} \mathrm{Zr}$ phase presents a closer pattern match.

Figure 44. (a) Bright field micrograph showing a grain, marked by a red arrow, selected for analysis (Grain 1). (b), (c), and (d) Electron diffraction analysis showing three selected zone axes collected from the grain, identifying the tetragonal- $\mathrm{H}_{2} \mathrm{Zr}$ phase (tI6, $\mathrm{I} 4 / \mathrm{mmm})$

Figure 45. (a) Bright field micrograph showing a grain, marked by a red arrow, selected for analysis; (b), (c), and (d) Electron diffraction analysis showing three selected zone axes collected from the grain, identifying the hexagonal- $\mathrm{UZr}_{2}$ phase (hP3, $\left.\mathrm{P} 6 / \mathrm{mmm}\right)$

Figure 46. Temperature profiles generated from TRIGA fuel annealing process at $1000^{\circ} \mathrm{C}$ for (a) 5 hours and (b) 24 hours. 46

Figure 47. Backscatter electron micrographs of (a) a selected region from the TRIGA fuel versus $304 \mathrm{SS}$ diffusion couple annealed at $1000^{\circ} \mathrm{C}$ for 5 hours, and (b) a detailed micrograph of the interaction region.

Figure 48. (a) Backscatter electron micrograph of interaction region in diffusion couple annealed for 5 hours, tentatively showing several phases and phase regions (PR). (b) through (e) Typical EDS patterns for the differing phase regions in the interaction region where (b) PR-1 is the 304SS away from the interaction region, (c) PR-2 is a U-Fe-Cr-Ni-rich phase in the interaction region, (d) PR-3 is a Fe-Cr-Ni-rich phase region, (e) PR-4 is a $\mathrm{Zr}-\mathrm{Fe}-\mathrm{Cr}-\mathrm{Ni}$-rich phase region, and (f) PR-5 is a U-Zr-Fe-Cr-Ni-rich phase region. 48

Figure 49. (a) Backscatter electron micrograph of the TRIGA fuel near the interaction region, in the diffusion couple annealed at $1000^{\circ} \mathrm{C}$ for 5 hours, tentatively showing three phases. (b) through (d) show typical qualitative EDS patterns for the differing phase regions (PR) in the interaction region where (b) PR-6 contains only Zr, (c) PR-7 is nearly pure U-phase, and (d) PR-8 is the fuel matrix.

Figure 50. Secondary electron micrograph displaying voids within TRIGA fuel near interaction region of diffusion couple.

Figure 51. (a) SE micrograph of the interaction region in the diffusion couple annealed at the $1000^{\circ} \mathrm{C}$ for 5 hours, and $\mathrm{x}$-ray maps of the region qualitatively showing the presence and location of (b) Er, (c) U, (d) $\mathrm{Zr}$, (e) Cr, (f) Fe, (g) Ni, (h) Co, (i) Cu, (j) Mn, (k) Mo, and (l) Si.

Figure 52. Backscatter electron micrographs of (a) a selected region from the TRIGA fuel versus $304 \mathrm{SS}$ diffusion couple annealed at $1000^{\circ} \mathrm{C}$ for 24 hours, and (b) a detailed micrograph of the interaction region.

Figure 53. (a) Backscatter electron micrograph of the interaction region, in the diffusion couple annealed at $1000^{\circ} \mathrm{C}$ for 24 hours, tentatively showing several phases. (b) through (e) show typical EDS patterns for the differing phase regions (PR) in the interaction region where (b) PR-1B is the 304SS away from the interaction region, (c) PR-2B is a $\mathrm{U}-\mathrm{Fe}-\mathrm{Cr}-\mathrm{Ni}$-rich phase in interaction region, (d) PR-3B is Fe-Cr-Ni-rich phase, and (e) $\mathrm{PR}-4 \mathrm{~B}$ is $\mathrm{Zr}-\mathrm{Fe}-\mathrm{Cr}-\mathrm{Ni}$-rich phase region.

Figure 54. (a) SE micrograph of the interaction region in the diffusion couple annealed at the $1000^{\circ} \mathrm{C}$ for 24 hours, and $\mathrm{x}$-ray maps of the region qualitatively showing the presence and location of (b) Er, (c) U, (d) Zr, (e) Cr, (f) Fe, (g) Ni, (h) Co, (i) Cu, (j) Mn, (k) Mo, and (1) Si. 
Figure 55. HAADF micrograph of the TEM sample obtained from the interaction region of the diffusion couple of TRIGA fuel versus 304SS annealed at $1000^{\circ} \mathrm{C}$ for 5 hours. The sample encompasses the entire thickness of the interaction region.

Figure 56. (a) Selected area of the interaction region showing the U-Fe-Cr-Ni-rich phase region (PR-2) in the interaction region. The EDS patterns in (b) through (f) show that the grains within this phase region contain distinct compositions with significant variations in $\mathrm{C}, \mathrm{Cr}, \mathrm{Fe}$, and $\mathrm{U}$

Figure 57. (a) Detailed micrograph of the elongated phase regions observed in the interaction region microstructure. The micrograph shows details from the region marked by a square in Figure 56. (b) through (g) show the differences in compositions of the specific grains labeled in (a).

Figure 58. (a) Detailed micrograph of the elongated phase regions observed in the interaction region microstructure. The EDS patterns in (b) and (c) show similar compositions to the EDS analysis in Figure 57, and show measurable Mo-concentrations.....

Figure 59. (a) Selected area of the interaction region showing the U-Zr-Fe-Cr-Ni-rich phase region (PR-5) in the interaction region. The EDS patterns in (b) through (f) show that the grains within this phase region contain distinct compositions with significant variations in $\mathrm{Cr}, \mathrm{Fe}, \mathrm{U}$, and $\mathrm{Zr}$.

Figure 60. (a) Detailed micrograph of the $\mathrm{Zr}-\mathrm{Fe}-\mathrm{Cr}-\mathrm{Ni}$-rich phase region (PR-4) near the interaction interface with TRIGA fuel. (b), (c) and (d) EDS patterns showing the grains with only $\mathrm{Zr}$ and $\mathrm{Zr}-\mathrm{Fe}-\mathrm{Cr}-\mathrm{Ni}$, and $\mathrm{Fe}-\mathrm{Cr}-\mathrm{Ni}$ compositions, respectively.

Figure 61. (a) Diffraction analysis of a selected grain within PR-2 in the interaction region. The diffraction patterns in (b) though (d) identify the grain as the cubic-UC phase.

Figure 62. (a) Diffraction analysis of a selected grain within PR-2 of the interaction region. The diffraction patterns in (b) though (d) identify the grain as the $\gamma$-Fe phase.

Figure 63. (a) Bright field micrograph of a grain selected from PR-5. EDS for the grain, shown in Figure 59(b) indicates that this grain contained significant concentrations of $\mathrm{U}, \mathrm{Fe}$, and Ni. Diffraction analysis, shown in (b) through (d), show that this grain was composed of the cubic- $\mathrm{Fe}_{2} \mathrm{U}$ phase.

Figure 64. U-Fe binary phase diagram. 


\section{TABLES}

Table 1. Measured concentrations of different observed features in fuel microstructure of sample annealed at $950^{\circ} \mathrm{C}$ for 5 hours.

Table 2. Measured concentrations of different observed features in fuel microstructure of sample annealed at $950^{\circ} \mathrm{C}$ for 16 hours.

Table 3. Measured concentrations of different observed features in fuel microstructure of sample annealed at $950^{\circ} \mathrm{C}$ for 24 hours.

Table 4. Measured concentrations of the different phase regions observed in the interaction region of the diffusion couple annealed at $1000^{\circ} \mathrm{C}$ for 5 hours, as labeled in Figure 48.

Table 5. Measured concentrations of the different phase regions observed in the TRIGA fuel near the interaction region of the diffusion couple annealed at $1000^{\circ} \mathrm{C}$ for 5 hours, as labeled in Figure 49.

Table 6. Measured concentrations of the different phase regions observed in the interaction region of the diffusion couple annealed at $1000^{\circ} \mathrm{C}$ for 5 hours, as labeled in Figure 53. 


\section{ACRONYMS}

304SS 304 stainless steel

BE backscatter electron

BF bright field

CAES Center for Advanced Energy Studies

EDS energy dispersive spectroscopy

Er Erbium

FCCI fuel cladding chemical interaction

FIB focused ion beam

$\mathrm{H}$ hydrogen

HAADF high-angle annular dark field

INL Idaho National Laboratory

PC personal computer

PR phase region

SAED selected area electron diffraction

SE secondary electron

SEM scanning electron microscopy

STEM scanning transmission electron microscopy

TEM transmission electron microscopy

TRIGA Training, Research, Isotopes, General Atomics

U uranium

UHP ultra-high purity

$\mathrm{Zr} \quad$ zirconium 


\section{High-Temperature Fuel Cladding Chemical Interactions between TRIGA Fuels and 304 Stainless Steel}

\section{INTRODUCTION}

High-temperature fuel cladding chemical interactions (FCCIs) between training, research, isotopes, general atomics (TRIGA) fuel elements and 304 stainless steel (304SS) are of interest to understand the fuel behavior during transient reactor scenarios. TRIGA fuels are composed of uranium (U) particles dispersed in a zirconium-hydride $(\mathrm{Zr}-\mathrm{H})$ matrix. In a reactor, the fuel is encased in 304SS or Incoloy 800 clad tubes. At high temperature, the fuel has the potential to interact with the cladding, resulting in FCCIs.

A number of FCCIs can take place in this system. Interactions can be expected between the cladding and $\mathrm{Zr}-\mathrm{H}$ matrix, and/or between the cladding and U-particles. Other interactions may be expected between the $\mathrm{Zr}-\mathrm{H}$ matrix and U-particles. Furthermore, the fuel contains erbium-oxide (Er-O) additions. Interactions can also be expected between the Er-O, cladding, $\mathrm{Zr}-\mathrm{H}$, and U-particles. The overall result is that very complex interactions may occur as a result of fuel and cladding exposures to high temperatures. Researchers at Idaho National Laboratory's (INL) Center for Advanced Energy Studies (CAES) conducted experiments to analyze these interactions.

Characterization of the baseline fuel (as-received, not irradiated or annealed) microstructure was performed to develop a detailed understanding of the fuel's initial microstructure. Fuel samples were then annealed at $950^{\circ} \mathrm{C}$ for 5,16 , and 24 hours to examine the microstructural evolution of the fuel at high temperature as a function of annealing time. Characterizations were carried out via scanning electron microscopy (SEM) and transmission electron microscopy (TEM) with sample preparation via a focused-ion-beam in situ lift-out technique. Compositional analyses were carried out by was energy dispersive spectroscopy (EDS) in both the SEM and TEM.

To develop a general understanding of element distributions in the baseline and annealed fuels, fuel samples were selected for characterization from each condition and were analyzed by SEM using secondary electron (SE) and backscatter electron (BE) imaging. Compositional analysis was carried out by EDS and x-ray mapping to examine the element distributions in each sample. Note that neither EDS nor x-ray mapping can detect the presence of hydrogen in the fuel.

Further characterization of the baseline and annealed microstructures was carried out by TEM to analyze in detail the different features observed in the SEM micrographs. For the annealed microstructures, only the sample annealed at $950^{\circ} \mathrm{C}$ for 24 hours was chosen for detailed TEM analysis. The samples were analyzed using bright field (BF) and high-angle annular dark field (HAADF) imaging with compositional analysis using the EDS detector in the TEM. Crystallographic analysis was performed by selected area electron diffraction (SAED) on specific grains within the microstructures, chosen based on composition and/or microstructural features. Just as in SEM, EDS in TEM cannot detect the presence of hydrogen in the fuel. Thus, the presence of hydrogen was inferred from the crystallographic analysis phase identification.

To examine FCCIs between the fuel and 304SS, diffusion couple experiments were carried out at $1000^{\circ} \mathrm{C}$ to develop interaction regions between the materials in laboratory controlled conditions. Diffusion couples consisting of TRIGA fuel versus 304SS were assembled and annealed at 5 and 24 hours. The diffusion couples developed interaction regions with complex microstructures that contained U-Fe-Cr-Ni, Fe-Cr-Ni, Zr-Fe-Cr-Ni, and U-Zr-Fe-Cr-Ni-rich phase regions. The interaction regions were characterized by the same methods used in the characterization of the fuel. 
The diffusion couple experiments revealed that the TRIGA fuel experienced shrinkage as a result of exposure to high temperature, likely the result of outgassing of hydrogen. An annealing experiment was carried out $1000^{\circ} \mathrm{C}$ for 24 hours to measure the loss of volume of the fuel at this temperature to correlate the results to those observed in the diffusion couples.

This report discusses the characterization of the baseline fuel microstructure and of the fuel after annealing at $950^{\circ} \mathrm{C}$ for 5,16 , and 24 hours. The effects on the microstructure of fuel due to exposure to high temperature are discussed. The results from the diffusion couple experiments carried out at $1000^{\circ} \mathrm{C}$ for 5 and 24 hours are presented with emphasis on the phase development of the interaction regions in each couple. The loss of volume of the fuel due to exposure to $1000^{\circ} \mathrm{C}$ for 24 hours is quantified.

\section{EXPERIMENTAL DETAILS \\ 2.1 Fuel Samples}

Fuel samples for this study were obtained from TRIGA fuel rod elements that had been sliced into disks approximately 1-1/8-in. diameter by 1/8-in. thick as shown in Figure 1(a). A disk was chosen randomly for further cutting. It was cut into $1 / 4$ in. $\times 1 / 4$ in. $\times 1 / 8$ in. pieces, as shown in Figure 1(b). Samples were prepared for characterization of the fuels, the annealing experiments and the diffusion couple studies. For each TRIGA fuel sample, both faces of the fuel samples were polished to a mirror finish using polishing steps with 400,600,800, and 1200-grit silicon carbide paper. The surfaces were polished in each step until the surface achieved a consistent surface roughness, and all prior scratches had been removed. The 1200 grit polishing was followed by 3-, 1-, and 1/4- $\mu \mathrm{m}$ polishing steps, each carried out for 10 min each, using alumina polishing paste.
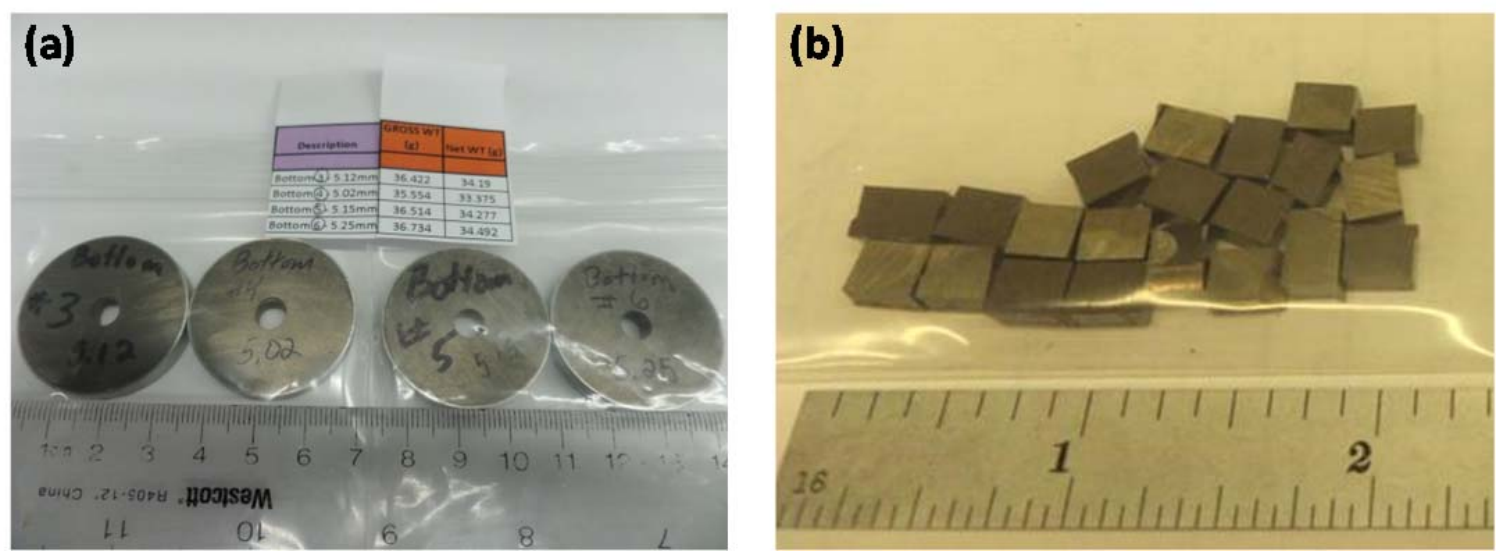

Figure 1. (a) TRIGA fuel disks used in this study; (b) fuel samples cut from a randomly selected fuel disk.

Two samples were selected for detailed characterization of the as-received microstructures. For these samples, only one of the faces was polished. Diffusion couple experiments were planned at 950 and $1000^{\circ} \mathrm{C}$. Three samples were used for annealing at $950^{\circ} \mathrm{C}$ for 5,16 , and 24 hours. Two samples were used for annealing at $1000^{\circ} \mathrm{C}$ for 5 and 24 hours. One sample was used to study fuel contraction as a result of exposure to high temperature.

\subsection{Equipment Setup}

To perform the annealing and diffusion couple experiments, a Paragon ${ }^{\mathrm{TM}}$ furnace model number SC-1 (Figure 2) was obtained. The furnace was selected so that it could be inserted into the radioactive materials glovebox at CAES through the loading port without the need for disassembly. 


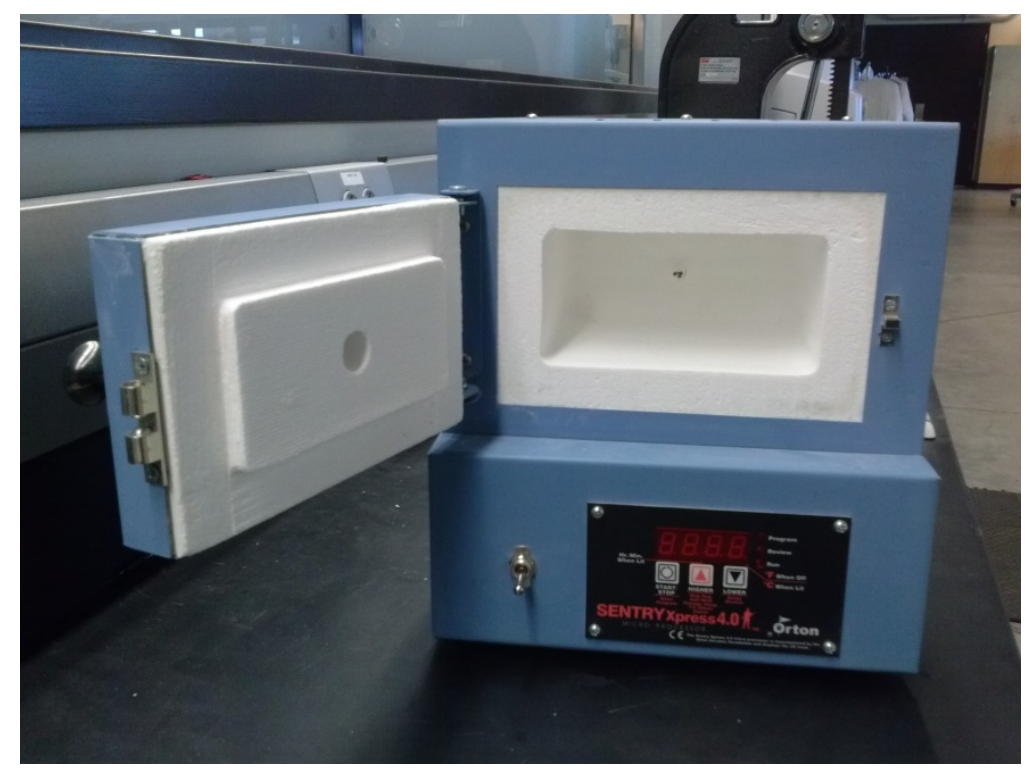

Figure 2. Box furnace utilized to carry out annealing and diffusion couple experiments.

The glovebox is normally operated using industrial-quality argon (Ar) gas. To reduce the amount of oxygen for the diffusion couple experiments, a higher purity Ar-gas feed line was inserted into the furnace through an available port on its door. A flow meter was placed on the Ar-gas feed line to measure the feed rate. The feed rate for the experiments was typically set to $0.5 \mathrm{ft}^{3} / \mathrm{hr}$. The glovebox is equipped with a gas inlet port, through which a gas cylinder was placed outside of the glovebox to supply the higher purity Ar gas.

Initially, ultra-high purity (UHP) Ar gas ( $<1 \mathrm{ppm} \mathrm{O}_{2}, 99.999 \%$ purity) was selected for the experiments. However, the initial diffusion couple experiments in the UHP argon-gas atmosphere suffered from significant oxidation during annealing. Therefore, in later diffusion couple experiments, BIP $^{\circledR}$ Technology argon gas $\left(<10 \mathrm{ppb} \mathrm{O}_{2}, 99.9999 \%\right.$ purity $)$ was obtained to minimize the oxygen impurity in the gas. To reduce the oxygen content even further, the argon gas was passed through a Supelco Analytical ${ }^{\mathrm{TM}}$ gas purifier. The final oxygen content in the argon gas was estimated at $1 \mathrm{ppb}_{2}$.

To monitor the furnace temperature, two thermocouples were inserted in the furnace. During annealing, the thermocouple ends were placed as close as possible to the samples in the furnace. For safety, two other thermocouples were placed in the glovebox, one directly above the furnace to monitor the outside furnace temperature and the other near the glovebox ceiling to monitor the glovebox temperature. The thermocouples were connected to wireless transmitters to avoid wiring through the glovebox walls. The transmitters were programed to send data at 5-sec intervals to a data logger connected to a standard personal computer (PC). The PC compiled the data into time-versus-temperature graphs. Figure 3 shows a schematic of the furnace setup inside of the glovebox. The schematic includes the Ar-gas tank setup outside of the glovebox. Figure 4(a) and (b) show the final furnace setup in the glovebox from two different angles. Figure 4(c) shows the Ar-gas cylinder with the inline gas purifier outside of the glovebox. Figure 4(d) shows a more detailed picture of the gas purifier. Figure 4(e) shows a picture of the Ar-gas tank label with the details of the gas. Note that the gas cylinder for BIP ${ }^{\circledR}$ Technology argon gas contains an internal purifier. 


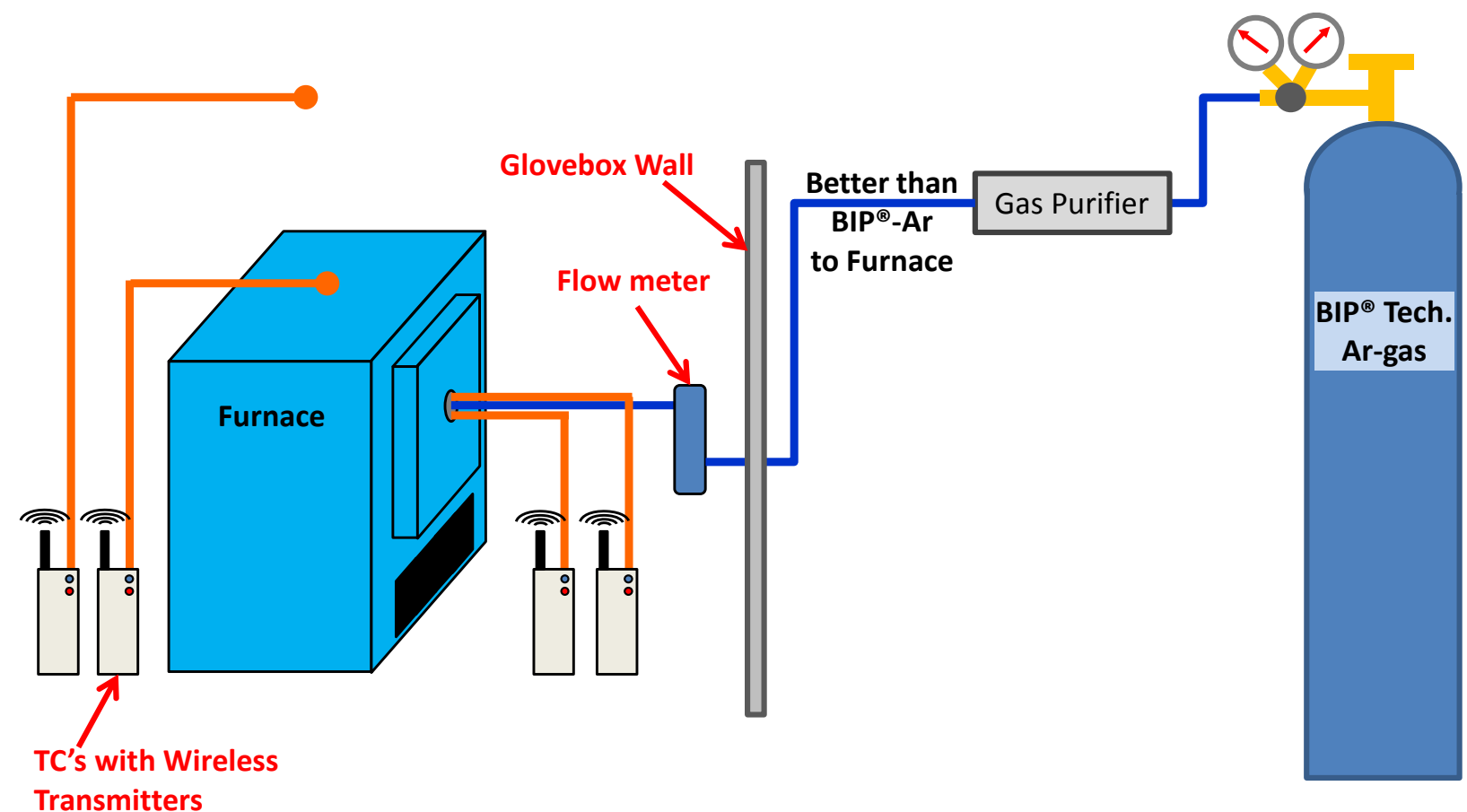

Figure 3. Schematic of the furnace setup inside of the glovebox including an Ar-gas tank external to the glovebox with an inline gas purifier. 

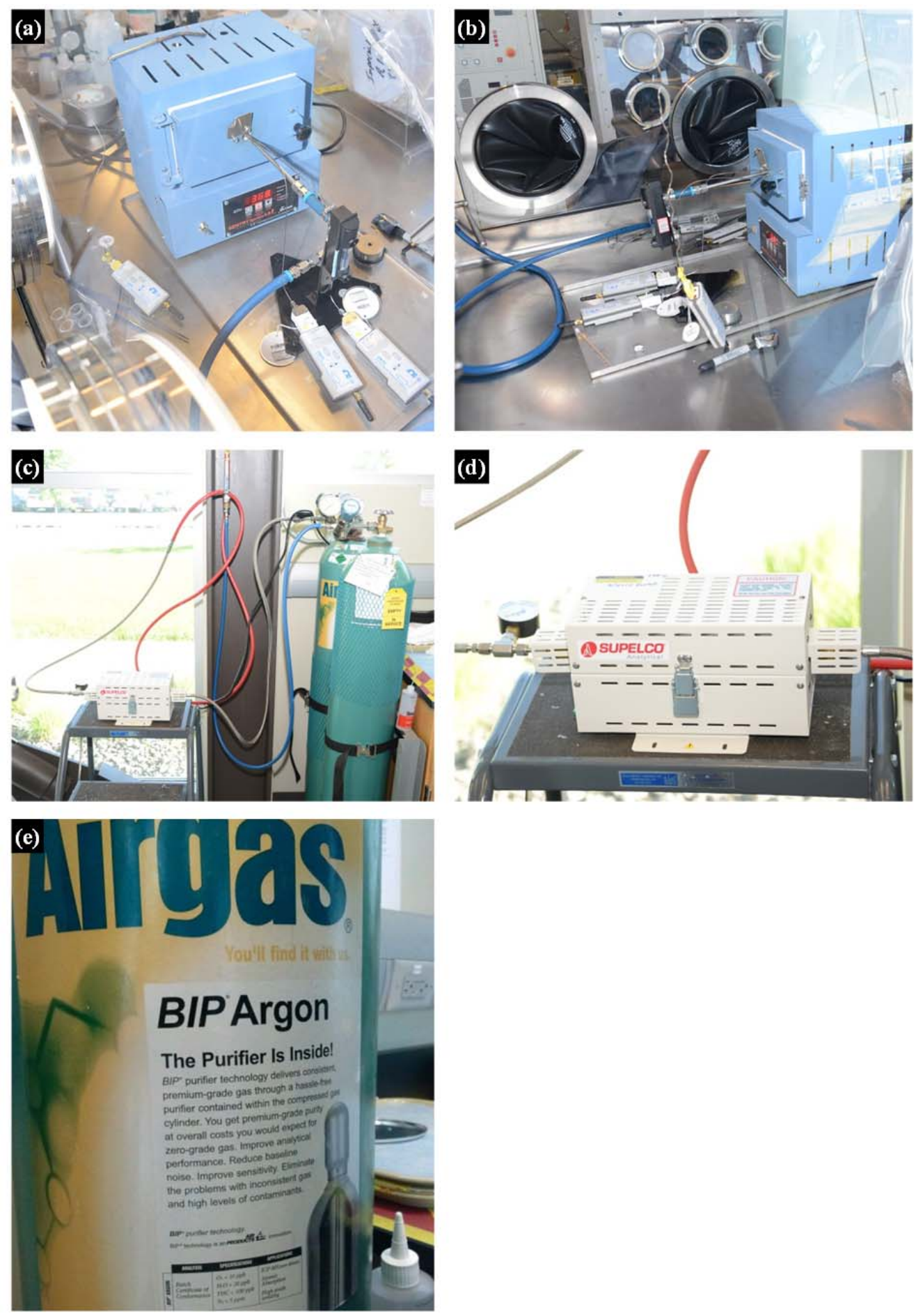

Figure 4. (a) and (b) The setup of the furnace inside of the glovebox from two different angles showing the Ar-gas feed line with a flowmeter, two thermocouples inserted through a port on furnace door, and two thermocouples placed outside of the furnace. (c) The Ar-gas line setup with a gas purifier inline.

(d) A more-detailed picture of the gas purifier. (e) A picture of the Ar-gas tank with the details of the gas. 


\subsection{Diffusion Couples and Fuel Annealing Experiments}

To examine FCCIs between TRIGA fuels and their 304SS cladding material, diffusion couple experiments were carried out to obtain interaction regions between the materials under laboratory-controlled conditions. Diffusion couples of TRIGA fuel versus 304SS were assembled and annealed for the experiments.

Initial anneals were carried out at $950^{\circ} \mathrm{C}$ for 5,16 , and 24 hours. Successful bonding of the couples was not achieved due to oxidation of the interaction interfaces during annealing. Thus, the TRIGA fuels from this diffusion couple experiments were utilized strictly to study the evolution of the fuel as a function of time due to exposure to high temperature. The root cause of the observed oxidation was determined to be the oxygen content of the UHP Ar-gas. This resulted in the upgrade in the quality of the gas to BIP ${ }^{\circledR}$ Technology Ar-gas.

Diffusion couples were then annealed at $1000^{\circ} \mathrm{C}$ for 5 and 24 hours. These couples successfully bonded for an indeterminate time period and developed interaction regions that were characterized in detail by SEM and TEM.

\subsubsection{Stainless Steel Material}

To perform diffusion couple experiments of TRIGA fuel versus 304SS, a 1/4-in. rod of 304SS was obtained from a commercial source. The rod was cut into 1/4-in.-thick pieces. One face from each piece used was polished using the same procedure addressed in Subsection 2.1 for the fuel.

\subsubsection{Diffusion Couple Jigs}

Jigs were used to hold diffusion couples together in the furnace. Different jig designs were used during the diffusion couple experiments. The original design, shown in Figure 5(a), was borrowed from a different project (note that the metals and temperatures in the image do not correspond to the current study). The second-generation design, shown in Figure 5(b), reduced the gap along the width in the jig to better grasp the TRIGA fuel and 304SS samples. In the third-generation design, Figure 5(c), one of the tightening screws was deemed unnecessary. In the fourth-generation design, shown in Figure 5(d), the jig was completely redesigned to (1) fit within the confines of a standard 1-1/4-in. epoxy mounting ring, so that the diffusion couple would not have to be manipulated off the jig for microstructural characterization, which reduced sample preparation labor, reduced the risk of damaging the diffusion couple, and increase reliability of the sample preparation process; and (2) the jig was designed to align the center of the diffusion couple cross section with the midpoint of the epoxy mounting ring so that the diffusion couple could easily be cross-sectioned precisely at the diameter of the diffusion couple. 

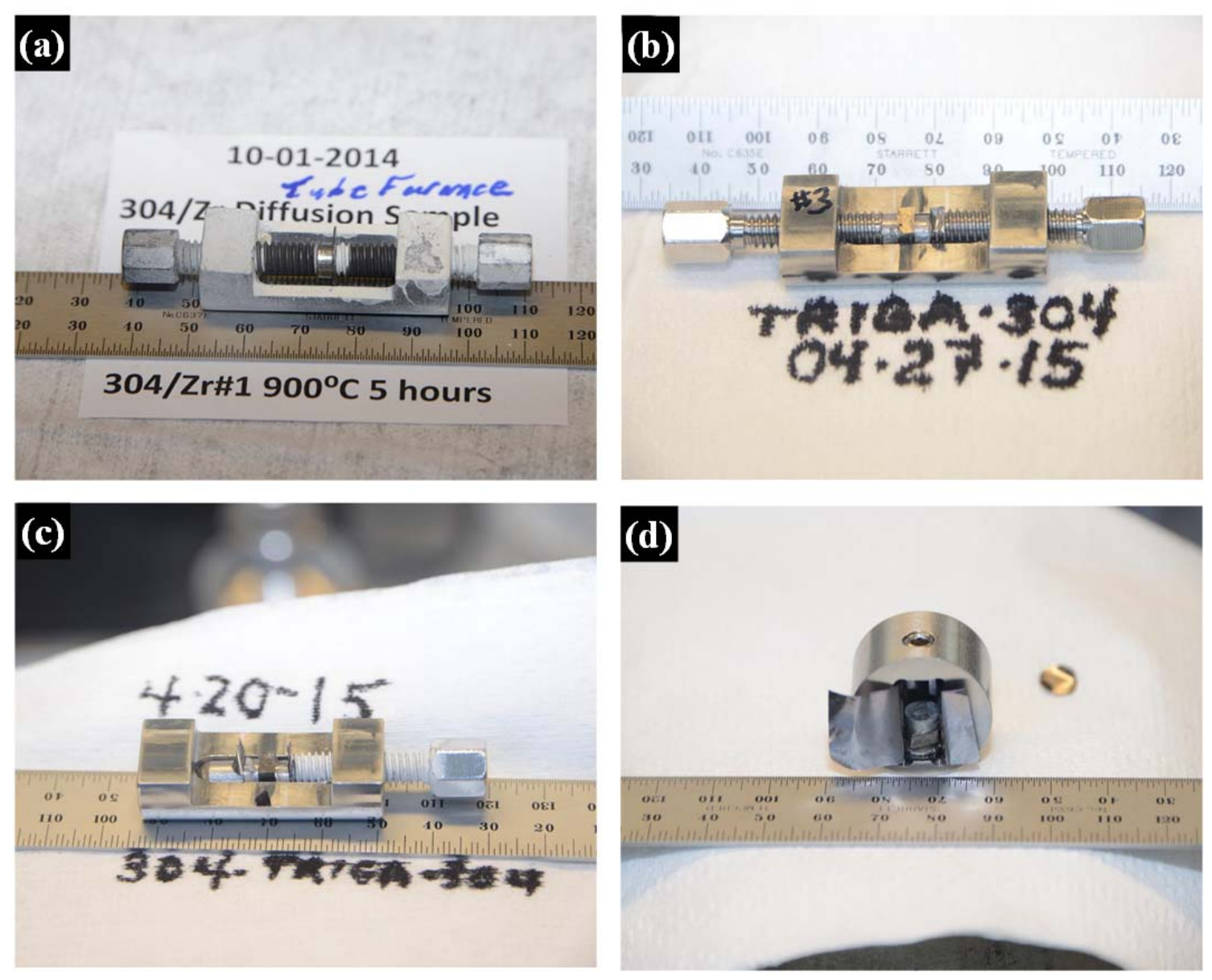

Figure 5. Four jig designs used to perform diffusion couple experiments. The jig designs are arranged from original to most recent design, $(\mathrm{a} \rightarrow \mathrm{d})$.

Preparation and annealing of all diffusion couple experiments were carried out inside of a glovebox that contained all the necessary materials to prepare and anneal the couples. After annealing, the diffusion couples were prepared for characterization within the same glovebox. The mounted and polished cross sections were then transferred out of the glovebox for characterization.

During preparation for annealing a diffusion couple in a jig, the TRIGA fuel and 304SS pieces were polished immediately prior to assembly to eliminate any oxidation that may have developed during storage. To assemble the diffusion couples, a fuel sample was placed between two 304SS pieces within the jig so that the polished surfaces could be brought into contact, as shown in the schematic in Figure 6 of a diffusion couple placed into a fourth-generation jig. The diffusion couples can also be observed in all jigs in Figure 5. 

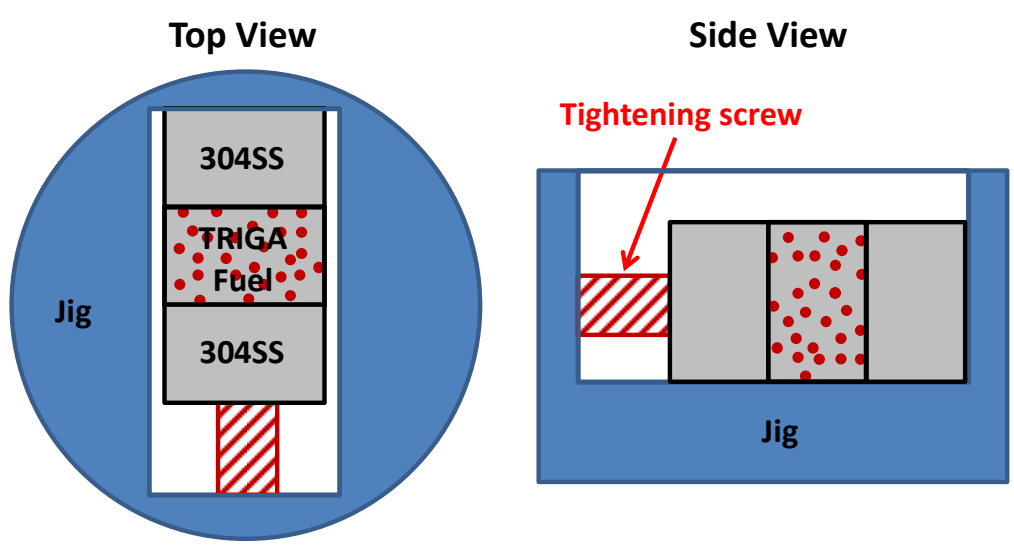

Figure 6. Schematic of a diffusion couple assembly onto a fourth-generation jig with top and side view perspectives.

After placing the fuel and 304SS pieces into the jig, they were brought together and tightened with the screws on the jig. During the initial experiments, the jigs were tightened by hand to avoid any significant loading pressure. In later experiments, the jigs were tightened to $30 \mathrm{in}$.-lb using a torque wrench. After tightening, the jigs were wrapped in three layers of tantalum foil, which served as an oxygen getter to further deter oxidation of the diffusion couples during annealing. Separate experiments determined that three layers of Ta-foil provided the required protection against oxidation within this experimental setup.

After a jig was assembled and prior to starting the furnace, the jig was placed as close as possible to the center of the furnace chamber. The furnace controller was programmed for the given anneal condition. The furnace door was closed, and the Ar-gas line and thermocouples were inserted into the furnace. The external thermocouples were set in place. The wireless thermocouples were set to transmit data. Data collection by the data logger was initiated. The gas purifier was then started and allowed to reach operating conditions. The Ar-gas flow into the furnace was then started, and it was allowed to flow for a minimum of 5 min to lower the oxygen content in the furnace chamber.

The furnace was then started and programed to heat at its maximum heating rate to achieve the annealing temperature as quickly as possible. After the desired annealing schedule was completed, the furnace was programed to decrease temperature at its maximum cooling rate, which exceeded the actual furnace cooling rate. Cooling typically took place over several hours. Quenching of the samples after annealing was not possible due to the restrictions of operating inside the radioactive materials glovebox. After the furnace had cooled sufficiently $\left(<100^{\circ} \mathrm{C}\right)$, the jigs were removed.

Initially, the diffusion couples were removed from the jigs for mounting. This resulted in damage to the diffusion couples, by separation at the contact surfaces. In later experiments, the diffusion couples were left in the jigs. When using first, second, or third-generation jigs, the jigs were cut around the diffusion couples so that they would fit in the epoxy mount. This step was unnecessary for the fourth-generation jigs. When using these jig designs, the entire jig was mounted in epoxy without disturbing the sample. After mounting, the diffusion couples were cross-sectioned and polished for microstructural characterization.

\subsubsection{Sample Characterization}

The samples were analyzed by SEM secondary and backscatter electron imaging. Compositional analysis in the SEM was carried out by EDS and x-ray mapping to develop a general understanding of the element distributions in the fuel and diffusion couples. Note that neither EDS nor x-ray mapping can detect the presence of hydrogen in the fuel. 
More detailed characterization was carried out by TEM with sample preparation via focused ion beam (FIB). TEM samples were selected based on the SEM results. TEM characterization was accomplished through BF and scanning transmission electron microscopy (STEM) HAADF imaging with compositional analysis by EDS. Crystallographic analysis was carried out by SAED on specific grains within the microstructure. A minimum of three zone axes were collected from each grain analyzed. Just as in the SEM, EDS in the TEM cannot detect the presence of hydrogen in the fuel. The presence of hydrogen was inferred from the crystallographic analysis phase identification.

Crystallographic analysis of the diffraction patterns collected in the TEM was carried with the JEMS Electron Microscopy Software Version 4.3010U2015. The crystallographic data was obtained from the Pearson's Crystal Data - Crystal Structure Database for Inorganic Compounds Release 2014/15.

Characterization of the baseline fuel and the fuels annealed at $950^{\circ} \mathrm{C}$ focused on the microstructural features in the fuel. The baseline fuel underwent characterization by SEM and TEM. All the fuels samples annealed at $950^{\circ} \mathrm{C}$ underwent characterization by SEM. Only the sample annealed for 24 hours underwent characterization by TEM.

The diffusion couples annealed at $1000^{\circ} \mathrm{C}$ were characterized by SEM and TEM using the same procedures applied to the fuel characterizations. However for the diffusion couples, only the interaction regions between the fuel and the 304SS were characterized in detail by TEM.

\section{RESULTS}

\subsection{Characterization of Baseline (As-Received) TRIGA Fuel 3.1.1 Characterization by Scanning Electron Microscopy}

TRIGA fuels are composed of U-particles dispersed in a $\mathrm{Zr}-\mathrm{H}$ matrix. Characterization of the TRIGA fuel by SEM analysis verified that the general microstructure of the fresh fuel consisted of particle dispersions, as shown in the BE micrographs in Figure 7(a) and (b). In these micrographs, the U-particles and the matrix appear in white and gray contrast, respectively. Figure 7(a) includes what appear to be grain boundaries near the center of the micrograph. The grain boundaries appear to be marked by a higher concentration of U-particles within the boundaries. Figure 7(b) shows large grains displaying dark-gray contrast, marked by red arrows, in the microstructure. Phase contrast in BE micrographs allows qualitative phase discrimination within a given microstructure. The deferring image contrast of the features shown in Figure 7(b) indicates that a distinct phase, differing from the general matrix and U-particles, is present within the fuel matrix. Figure 7(b) also displays a small void in the microstructure, marked by a red arrow. 

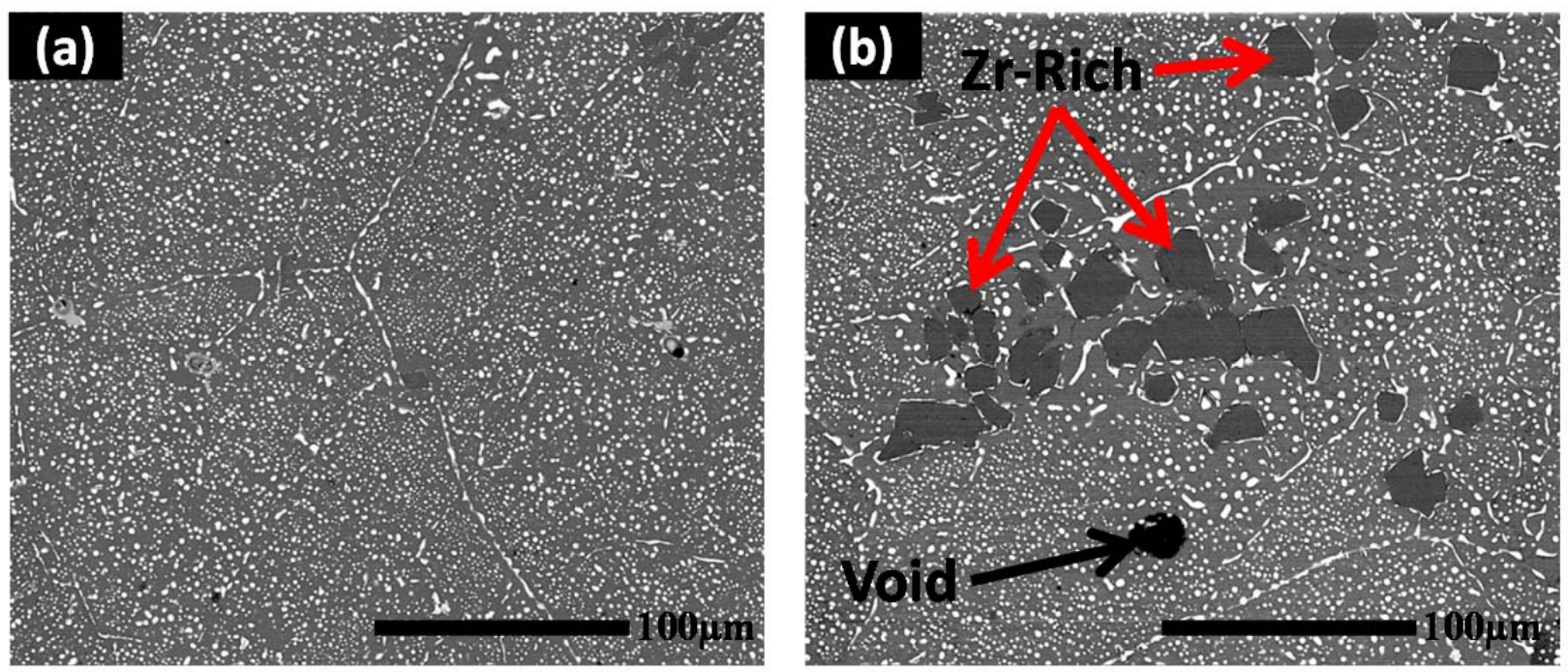

Figure 7. Backscatter electron micrographs showing the typical microstructure of fresh TRIGA fuel analyzed in this study. (a) Emphasizes the general microstructure and apparent grain boundaries.

(b) Typical dark contrast grains found throughout the microstructure.

BE micrographs in Figure 8 show the fuel microstructure in greater detail. Figure 8(a) details the fuel microstructure, including a grain boundary and a grain from the dark contrast phase similar to those seen in Figure 7(b). Figure 8(a) shows that the U-particles within the matrix are generally spherical in shape (extrapolating to three dimensions), while those at the grain boundaries show elongated microstructures. Figure $8(\mathrm{~b})$ shows, based on phase contrast, that yet another phase is present within the grain boundaries as marked by the white arrows.
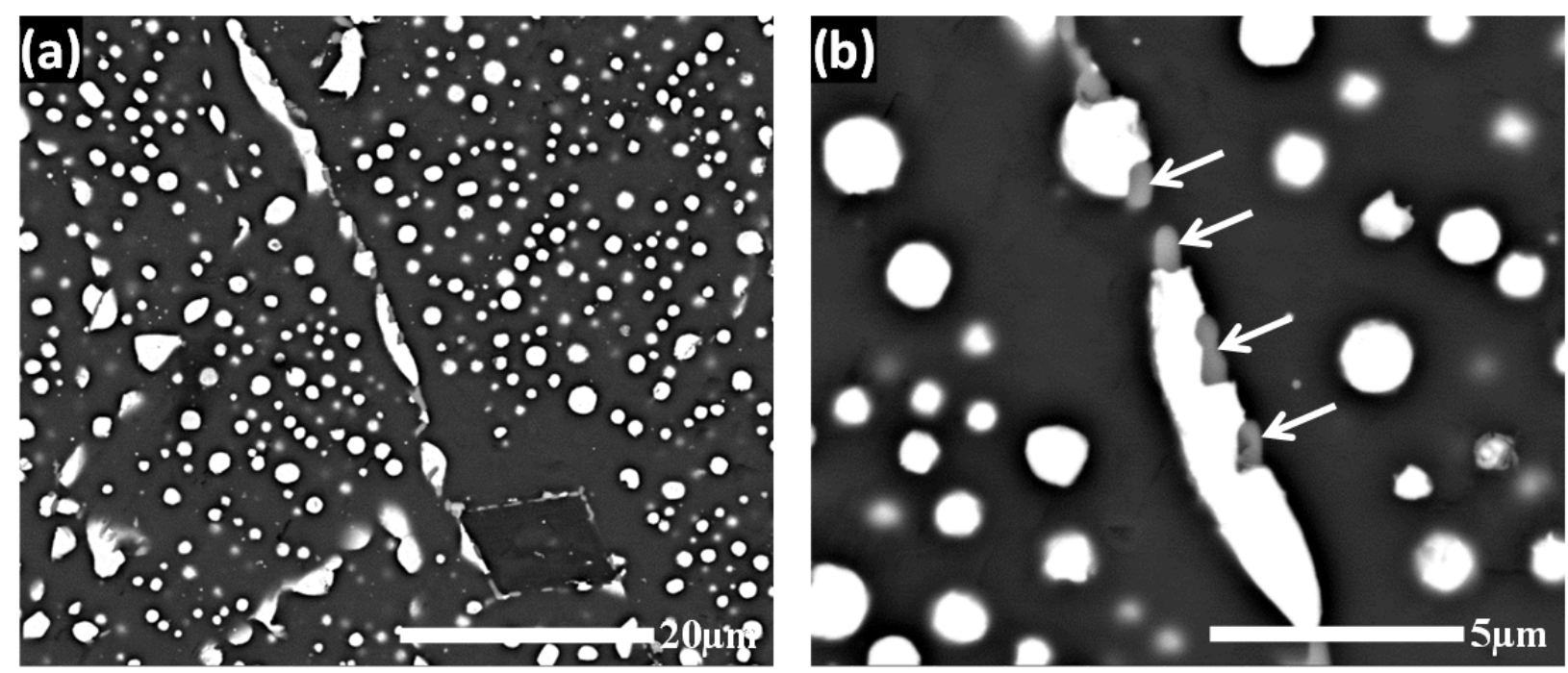

Figure 8. BE micrographs showing (a) the fuel microstructure with a grain boundary included, and (b) a detailed section of a grain boundary containing $U$ and another distinct phase (marked by white arrows).

EDS analysis was performed to develop an understanding of the element distributions and compositions of the phases shown in the SEM BE micrographs in Figure 7 and Figure 8. EDS cannot detect hydrogen; thus, the analysis was limited to a general assessment of the $\mathrm{Zr}, \mathrm{U}$, and other element distributions within the fuel matrix. Figure 9 shows the EDS patterns of the different microstructural 
features shown in the SEM BE micrographs. Figure 9(a) shows the matrix composition (not including hydrogen); it shows that the matrix did not include any measurable concentrations of $U$ or any other element. Figure 9(b) shows that U-particles did not contain Zr, but appear to contain a measurable carbon (C) concentration (possible hydrogen content was not measured). Figure 9(c) shows the measured EDS pattern from the dark contrast phase grains shown in Figure 7(b). Because EDS cannot detect hydrogen, the EDS patterns shown in Figure 9(a) and Figure 9(c) appear identical. However, based on phase contrast, the hydrogen content may vary between the matrix and the dark contrast grains. Figure 9(d) shows an EDS pattern obtained from the phase observed within the grain boundaries shown in Figure 8(b). EDS identified the presence of Er and $\mathrm{O}$ within this phase. Note that the particles are relatively small compared to the interaction volume of the electron beam in the SEM. The EDS pattern in Figure 9(d) shows significant $U$ and $\mathrm{Zr}$ peaks. These peaks may result from beam interactions with the adjacent matrix and U-particles. Therefore, the measured particles may not contain these elements.
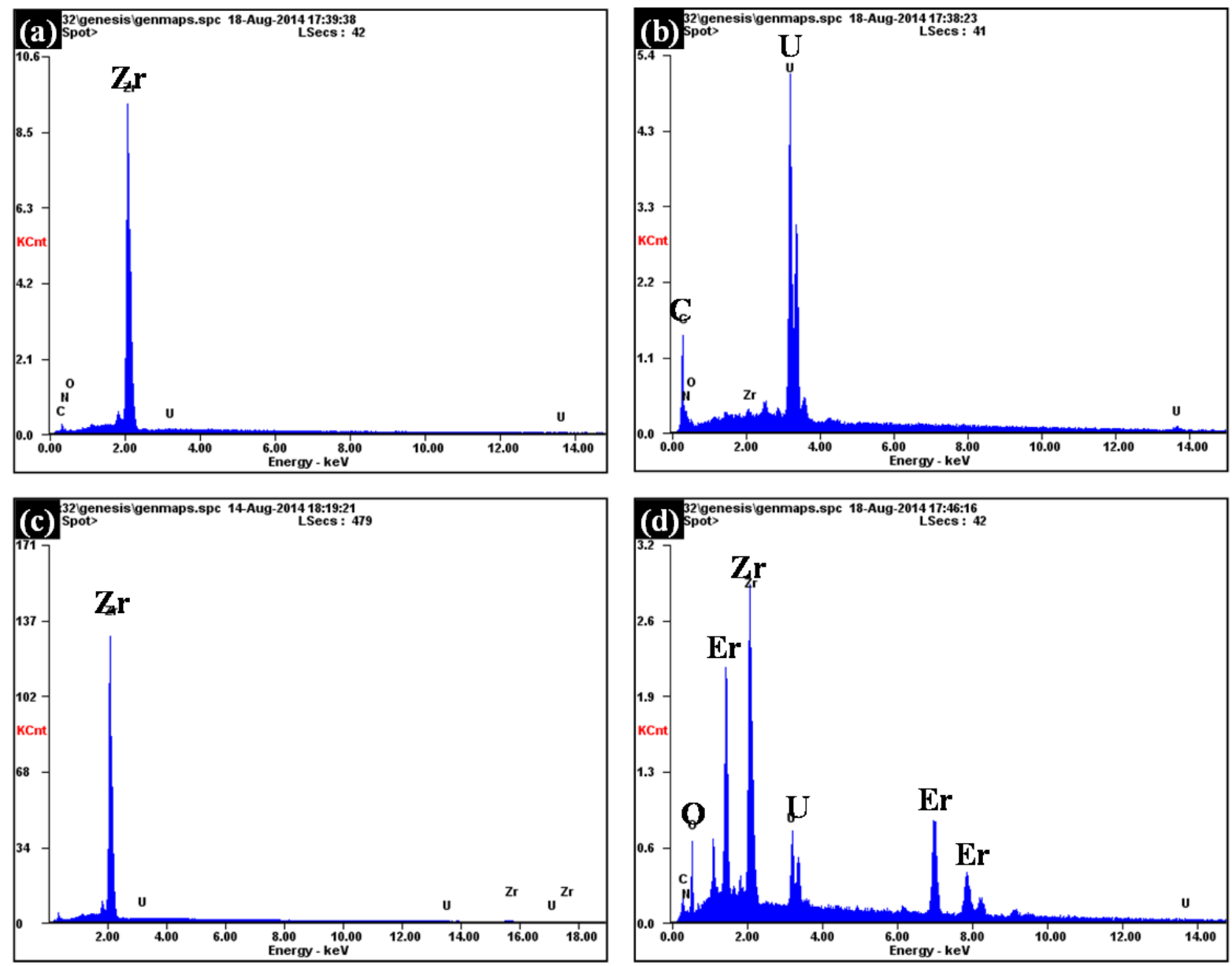

Figure 9. Energy dispersive spectroscopy patterns of the (a) matrix, (b) U-particles, (c) dark contrast grains, and (d) the phase observed within the grain boundaries.

To obtain a visual assessment of the element distributions in the fuel, $\mathrm{x}$-ray maps were generated from selected regions of the fuel. X-ray maps qualitatively show element concentrations in a given region as intensities of color in the image. Unless enhanced, higher color intensities denote greater number of detections and generally suggest higher concentrations of the element under analysis. The SE micrograph in Figure 10(a) shows the region selected for analysis. Figure 10(b) shows the Zr distribution in the fuel. The dark or black features in the image mark the locations of the U-particles, and show that $\mathrm{Zr}$ is not present within the particles. Figure 10(b) also shows higher color intensity in the regions where the dark 
contrast features shown in Figure 7(b) are located, suggesting that these features contain a higher concentration of $\mathrm{Zr}$ than the matrix. Figure 10(c) confirms the location of the U-particles, and indicates the matrix lacks U. Figure 10(d) shows that Er may be restricted to the grain boundaries. Figure 10(e) shows that oxygen concentration is high where Er is present in the microstructure. This may indicate that Er is present as an Er-O within the grain boundaries.
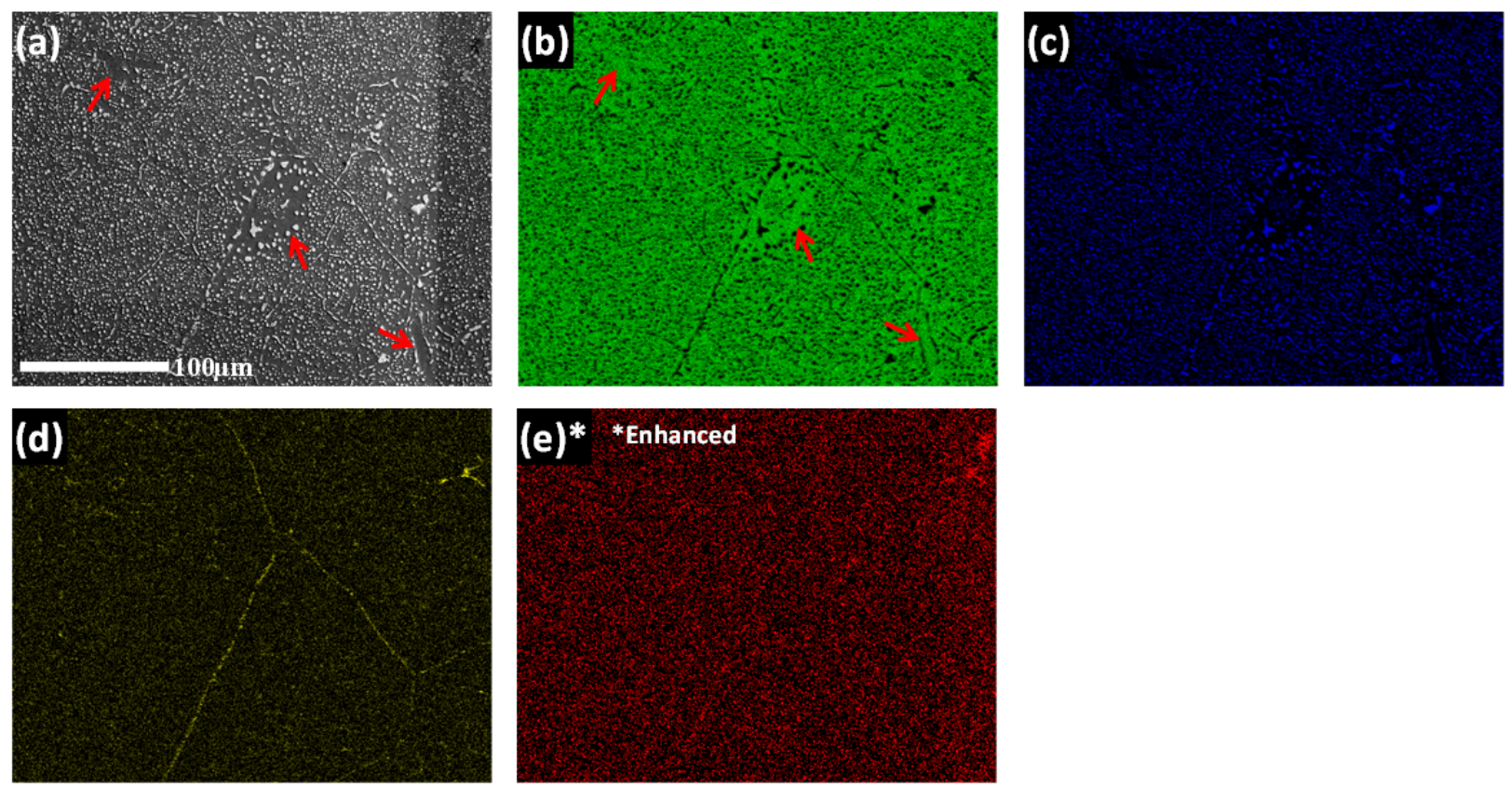

Figure 10. X-ray maps of a selected region of the TRIGA fuel showing (a) a secondary electron micrograph of selected region of TRIGA fuel for x-ray mapping; x-ray mapping of (b) Zr, (c) U, (d) Er, and (e) $\mathrm{O}$ intensities. (Note: The oxygen intensity was enhanced to emphasize oxygen concentration in the grain boundaries.)

\subsubsection{Characterization by Transmission Electron Microscopy}

Further characterization of the TRIGA fresh fuel was carried out by TEM to examine the microstructure in greater detail and to characterize the phases present in the fresh fuel through diffraction analysis. Five TEM samples were required to capture the different features observed by the SEM. Figure 11 shows a HAADF micrograph of each sample. Figure 11(a) shows the general microstructure that included U-particles in the Zr-H matrix. Figure 11(b) and Figure 11(c) show grain boundaries that contain Er-grains. Figure 11(d) and Figure 11(e) captured Zr-rich grains similar to those shown in Figure $7(b)$ as the dark contrast phase. 

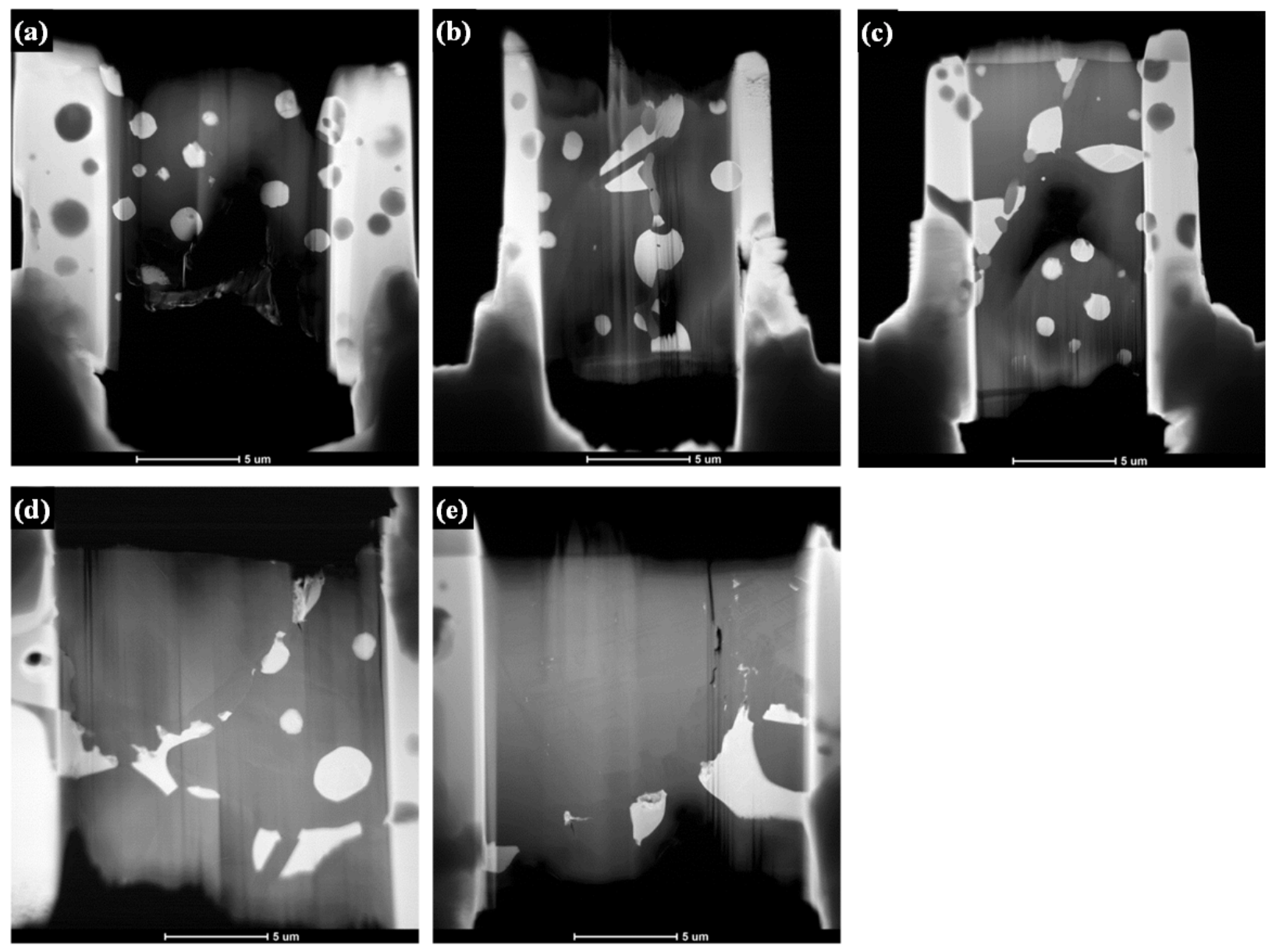

Figure 11. HAADF micrographs of five TEM samples prepared to capture the different microstructural features found in the fresh TRIGA fuel: (a) general microstructure, (b) and (c) grain boundaries with Er-grains, and (d) and (e) Zr-rich phase (dark contrast phase in Figure 7[b]).

The HAADF micrographs in Figure 12(a) and Figure 12(b) show the typical microstructure of the U-particles in the matrix. Based on image contrast, interaction regions appear to have developed between the U-particles and the Zr-H matrix. Figure 12(c) and Figure 12(d) show typical EDS patterns of the Zr-H matrix and the U-particles, respectively. Figure 12(e) supports that interaction regions are present, as the gray contrast regions in the images contain both $U$ and $Z r$. However, the compositions observed in Figure 12(e) can also indicate overlapping U- and Zr-grains in the sample. Note that the interaction regions may also contain significant concentrations of hydrogen that are not detectable by EDS. 

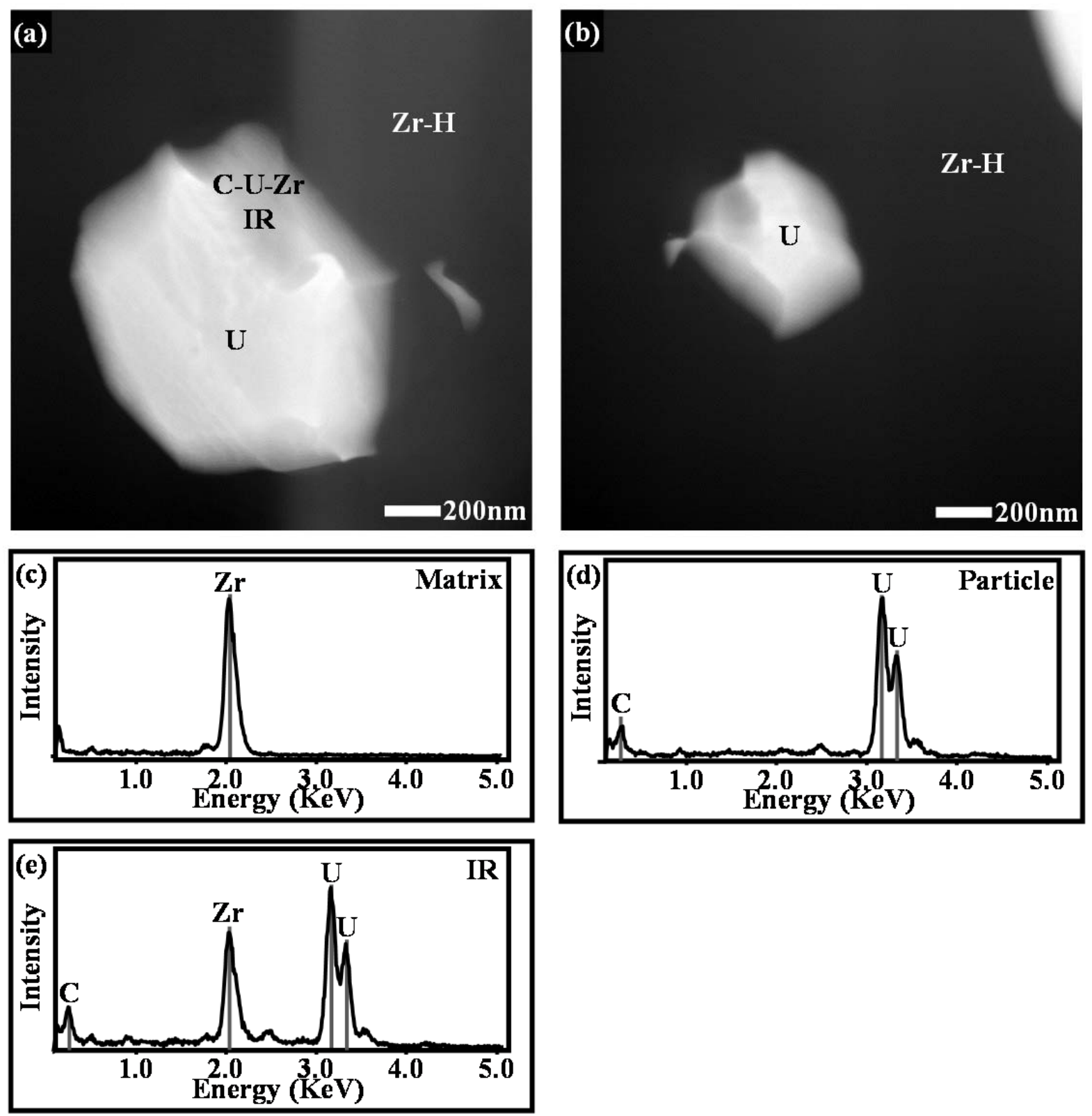

Figure 12. (a) and (b) Typical high-angle annular dark field micrographs of U-particles in the $\mathrm{Zr}-\mathrm{H}$ matrix displaying likely interaction regions with the corresponding EDS patterns of the (c) Zr-H matrix, (d) unreacted U-particles, and (e) C-U-Zr interaction region between $\mathrm{U}$ and $\mathrm{Zr}-\mathrm{H}$.

Similarly, the HAADF micrographs in Figure 13(a) and (b) show typical HAADF micrographs of regions containing Er-grains. Figure 13(c) shows a typical EDS pattern of the Er-grains. This pattern shows that the Er-grains contain significant concentration of oxygen and do not contain measurable concentrations of $\mathrm{U}$ or $\mathrm{Zr}$. Interaction regions are apparent between the U-particles and the Er grains and between the Er grains and the Zr-H matrix. Figure 13(d) and (e) show that these interaction regions contain Er-O-U and Er-O-Zr compositions, respectively. 

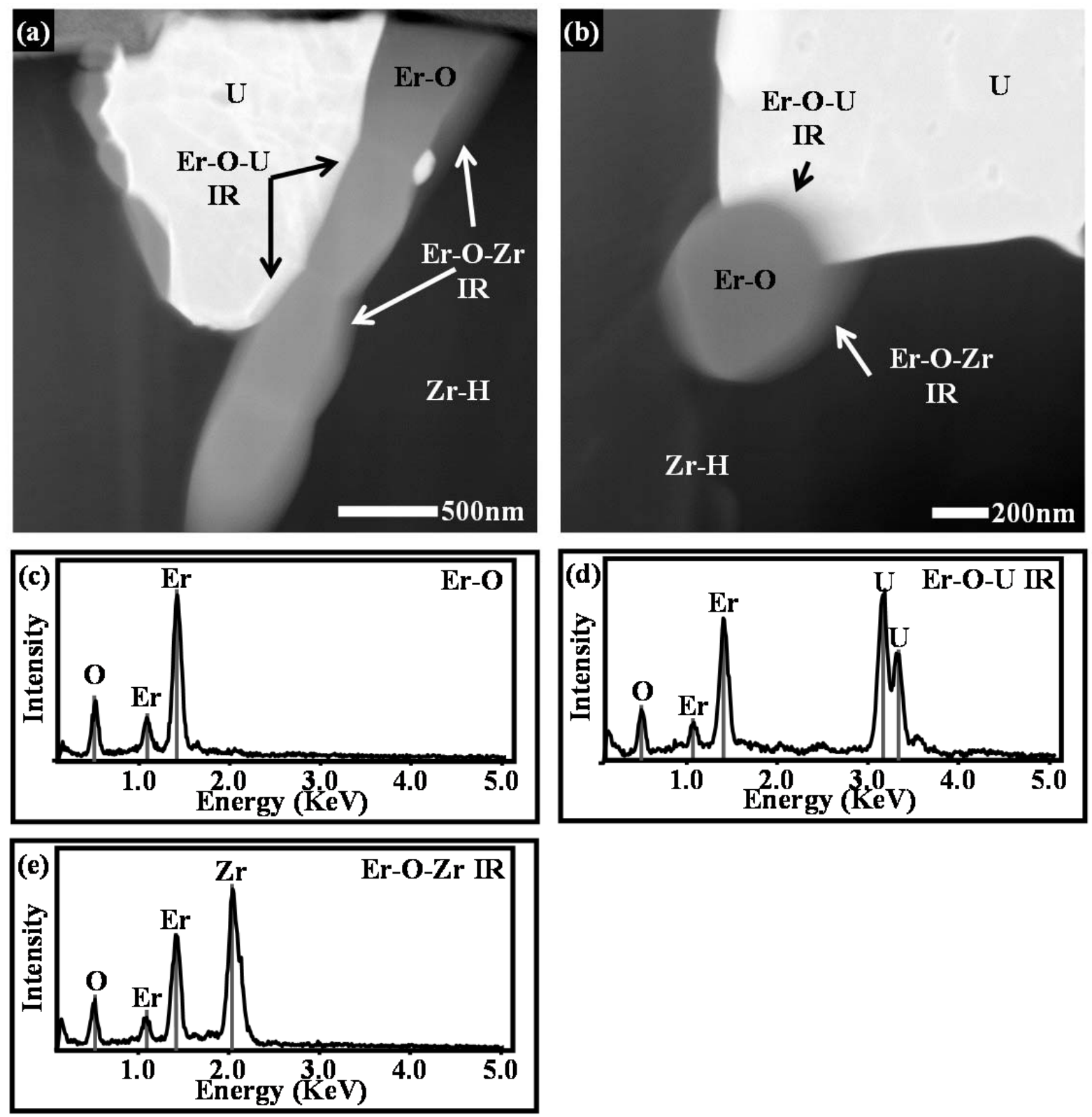

Figure 13. (a) and (b) HAADF micrographs of typical $\mathrm{U}$ and Er-O particles in the $\mathrm{Zr}-\mathrm{H}$ matrix showing displaying likely Er-O-Zr and Er-O-U interaction regions with the corresponding EDS patterns of the (c) Er-O, (d) Er-O-U , and (e) Er-O-Zr interaction regions.

The Zr-rich microstructural features, which were observed in the fuel matrix that exhibited a dark contrast in the SEM BE micrographs and introduced in Figure 7(b), were examined in more detail by TEM. The HAADF micrographs in Figure 14(a) through (d) detail the microstructures of these features at different magnifications. These micrographs show, based on phase contrast, that the features appear to contain at least two phases with lamellar microstructures. The red arrows in the figures mark the boundary between the matrix and these features. The BF micrographs in Figure 15 emphasize a complex and fine-grained lamellar microstructure. 

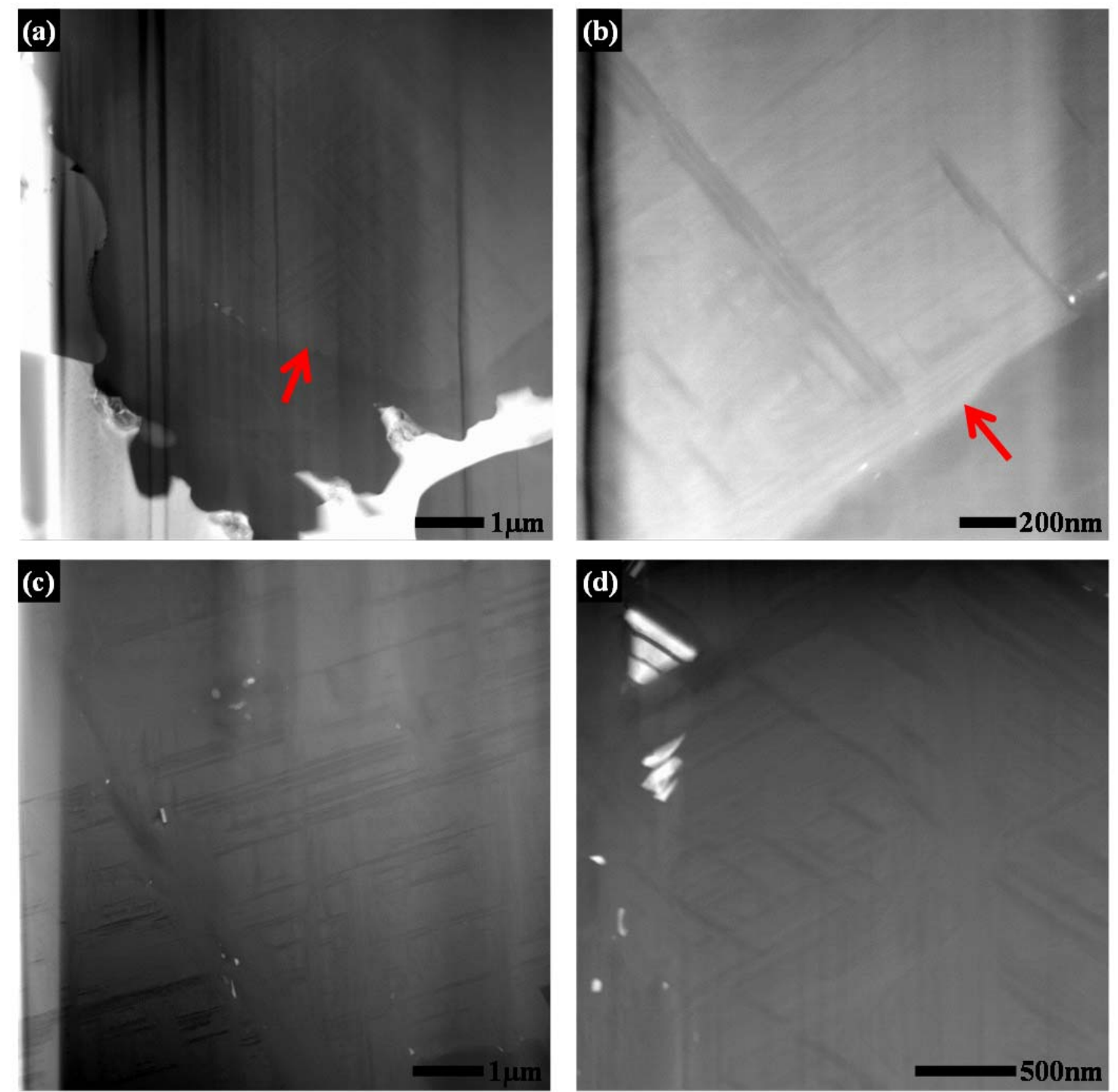

Figure 14. HAADF micrographs (a) through (d) at increasing magnifications of the secondary Zr-rich particles observed in fuel matrix microstructure.
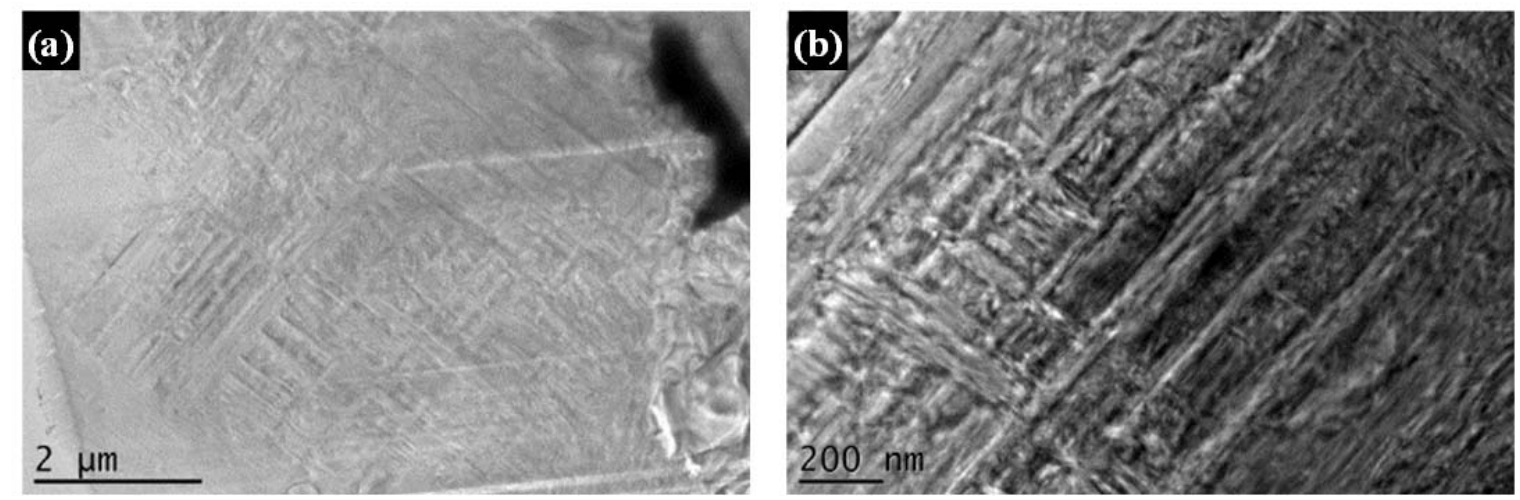

Figure 15. BF micrographs (a) and (b) at increasing magnification of the secondary Zr-rich particles observed in fuel matrix microstructure emphasizing microstructural features. 
Concentration profiles were generated from the interaction regions to develop an initial understanding of the interactions between the elements. Figure 16 through Figure 18 show the (a) HAADF micrographs with the selected regions for analysis and (b) the generated concentration profiles. The concentration profiles were collected within the red lines on each HAADF micrograph. The dashed lines on the micrographs mark the regions where interactions are apparent based on observation of the profiles. Note that due to the limits of the EDS equipment, carbon and oxygen cannot be reliably quantified. Thus, the shown concentration profiles may contain significant error. Even though the EDS patterns in Figure 13(d) and (e) strongly suggest that carbon is present in the U-particles, it was not included in the concentration profiles due to a significant quantification error. Nonetheless, the crystallographic analysis below considers its presence.

Figure 16(a) shows a likely interaction region between a U-particle and the $\mathrm{Zr}-\mathrm{H}$ matrix. The concentration profile in Figure 16(b) shows that the elements experienced relatively consistent concentration gradients through the region of interaction. Near the U-rich side of the profile (approximately at position $175 \mathrm{~nm}$ ), a change in the profile gradient is observed. This may indicate the position of a phase change within the interaction region.

Figure 17(a) shows a selected apparent interaction region between an Er grain and the $\mathrm{Zr}-\mathrm{H}$ matrix. Figure 17(b) shows the collected concentration profile from the marked region. The profile shows continuous gradients throughout the interaction region. The gradients in the profile closely correspond to the contrast change observed in the micrograph. Er, $\mathrm{O}$, and $\mathrm{Zr}$ are present throughout the interaction regions.

Figure 18(a) shows a U-particle in contact with an Er grain. Although image contrast does not clearly suggest the presence of an interaction region, the concentration profile in Figure 18(b) shows that significant interactions appear to take place. The profile shows, as marked in Figure 18(a), that the interaction region is developed largely within the U-particle. Little-to-no U was measured within the Er grain. Also, the concentration profile shows that Er penetrated farther into the $U$ than oxygen. Note that the interaction volume of the electron beam in the TEM is significantly smaller than the length of the measured interaction region. As a result, the interaction volume of the electron beam that may skew the data can be ruled out as the cause of the observed concentration gradients. 

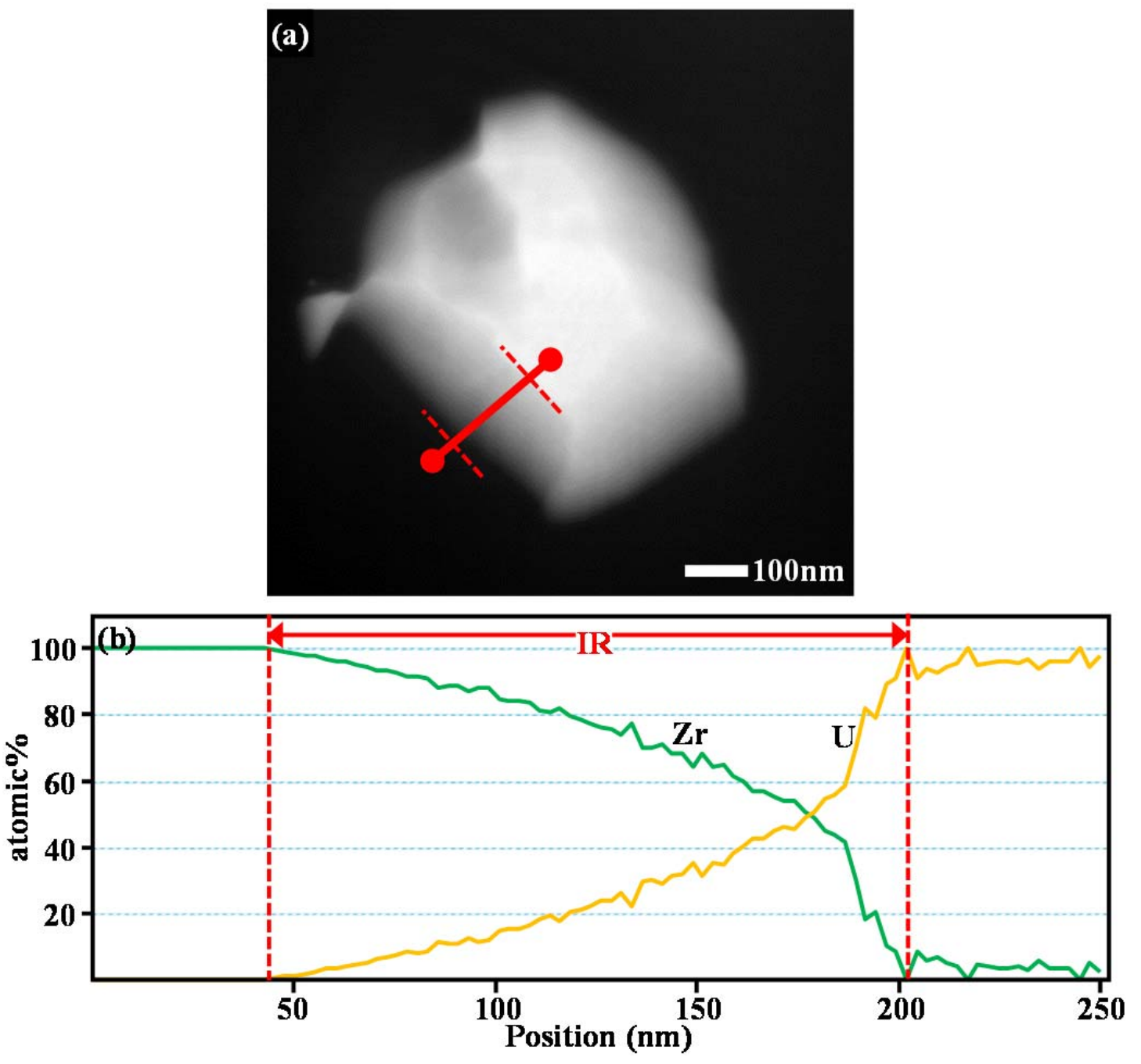

Figure 16. (a) HAADF micrograph of a selected interaction region between a U-particle and the $\mathrm{Zr}-\mathrm{H}$ matrix. (b) The concentration profile collected at the red line, and between the dashed lines, shown in (a). 

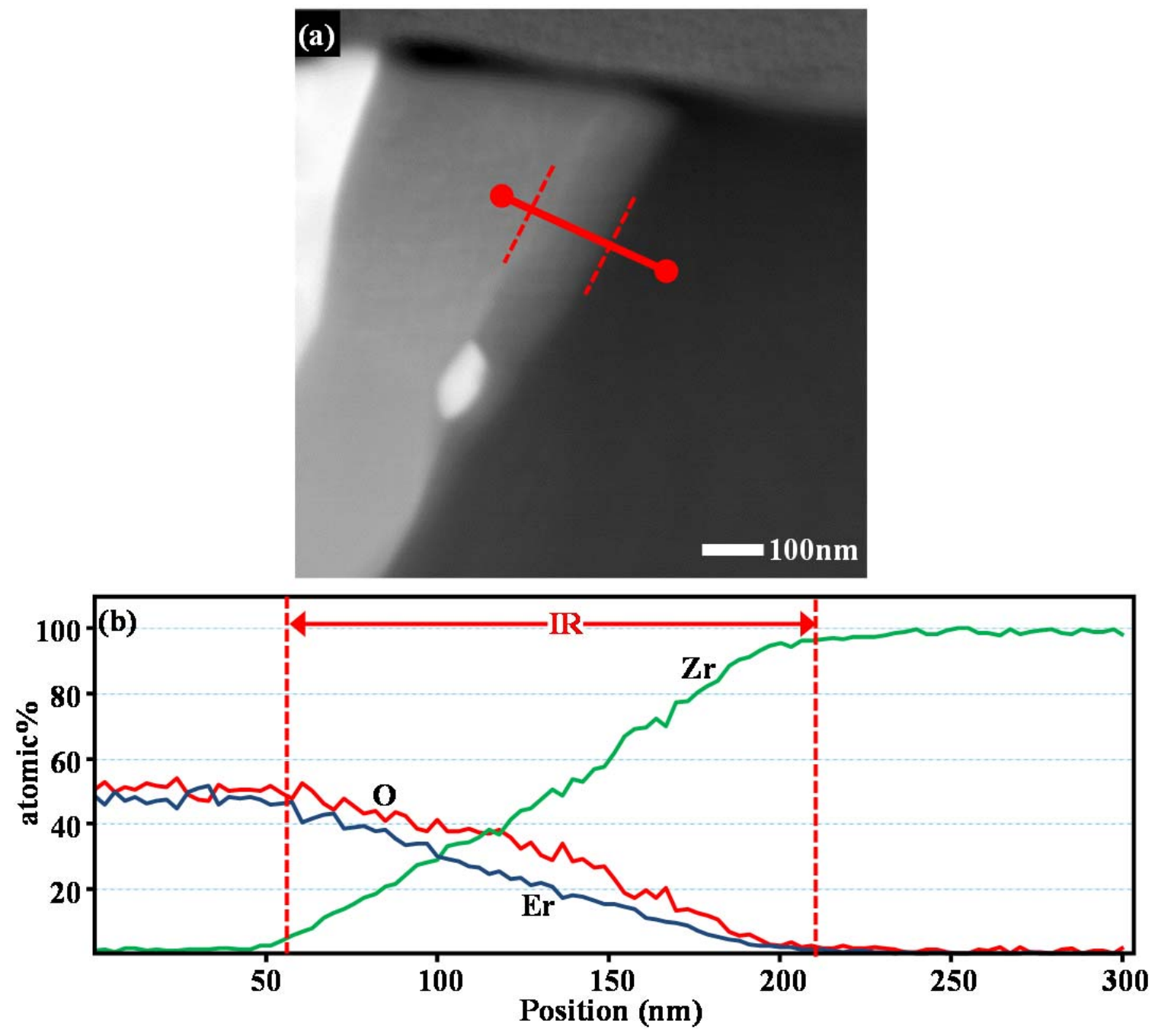

Figure 17. (a) HAADF micrograph of a selected interaction region between an Er grain and the $\mathrm{Zr}-\mathrm{H}$ matrix. (b) The concentration profile collected at the red line, and between the dashed lines, shown in (a). 

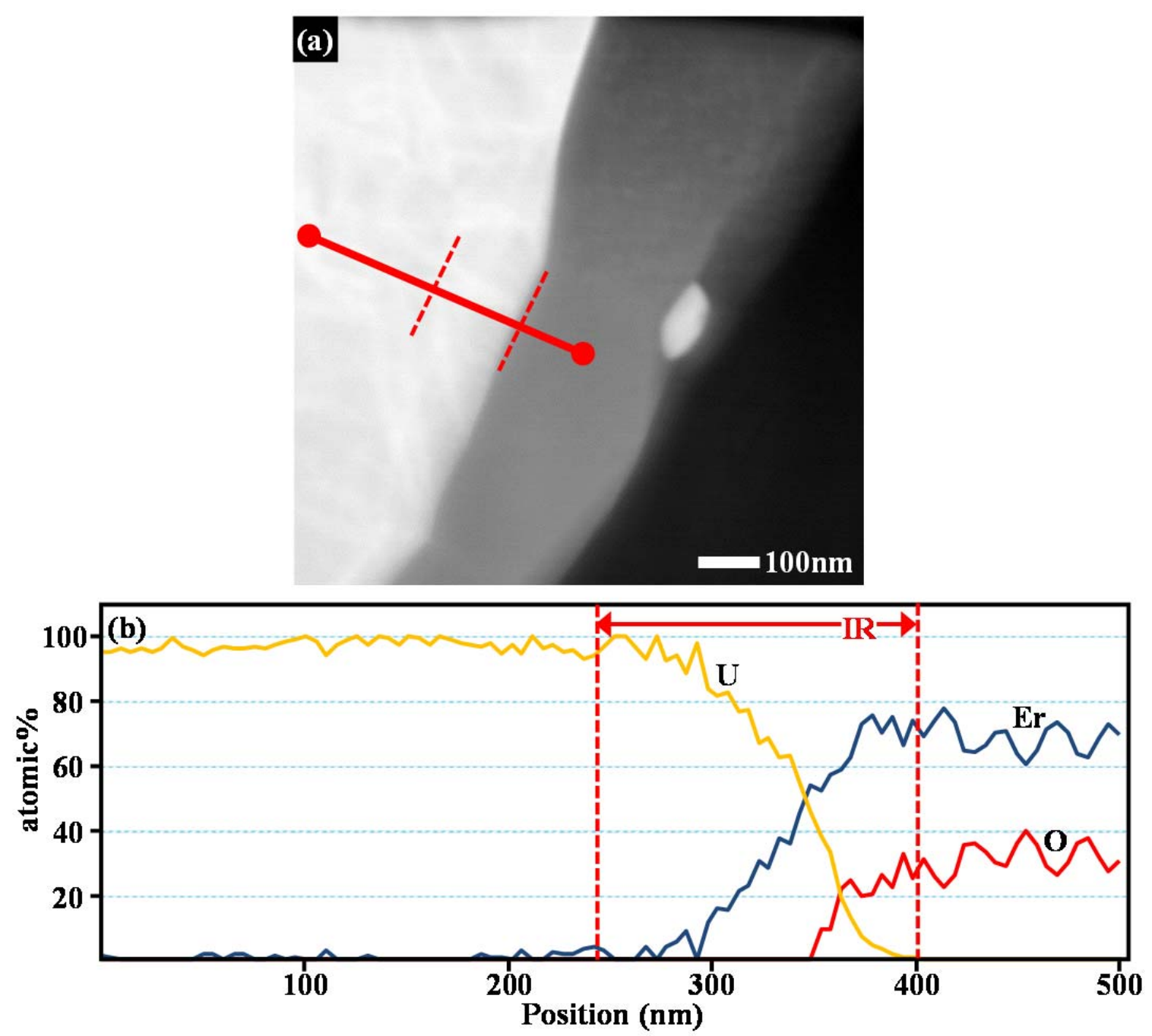

Figure 18. (a) HAADF micrograph of a selected interaction region between a U-particle and an Er grain; (b) The concentration profile collected at the red line, and between the dashed lines, shown in (a).

To fully characterize the TRIGA fuel, characterization by crystallographic analysis of electron diffraction patterns was performed on the fuel matrix, U-particles, Er grains, dark contrast phase (SEM), and the observed interaction regions. Diffraction analysis allows determination of the crystallographic nature of a specific grain or region within the microstructure to identify the phase or phases present. Because EDS cannot detect hydrogen, diffraction analysis was performed with the assumption that hydrogen was present in all of the features observed in the fuel. If a given phase under analysis was found to fit a given hydrogen-containing phase, and not a phase lacking hydrogen, then the phase was identified as containing hydrogen. In this manner, the presence of hydrogen in the system was determined. To attain confidence in the analysis of the fuel, several grains from each observed feature were analyzed. To summarize the results, this report includes only one grain from each feature.

The BF micrograph in Figure 19(a) shows a grain (marked by red arrow) that was selected from the $\mathrm{Zr}-\mathrm{H}$ matrix for diffraction analysis. The electron diffraction patterns in Figure 19(b) through Figure 19(d) show three different zone axes from the selected grain. The patterns match the tetragonal $\mathrm{H}_{2} \mathrm{Zr}$ phase (tI6, I4/mmm). Based on the literature [1-5], TRIGA fuels normally contain the cubic- $\mathrm{ZrH}_{1.6}$ phase as the matrix. Although the $\mathrm{ZrH}_{1.6}$ phase presented a close match to the diffraction patterns, the $\mathrm{H}_{2} \mathrm{Zr}$ phase presented a better fit. 
Note that a significant portion of the spot patterns from this and other analyzed grains (not shown) contained unidentified reflections that cannot necessarily be explained by single crystal diffraction. Possible explanations for these unidentified reflections in the fresh fuel are given at the end of this section. However, further analysis is required to reach more conclusive explanations.
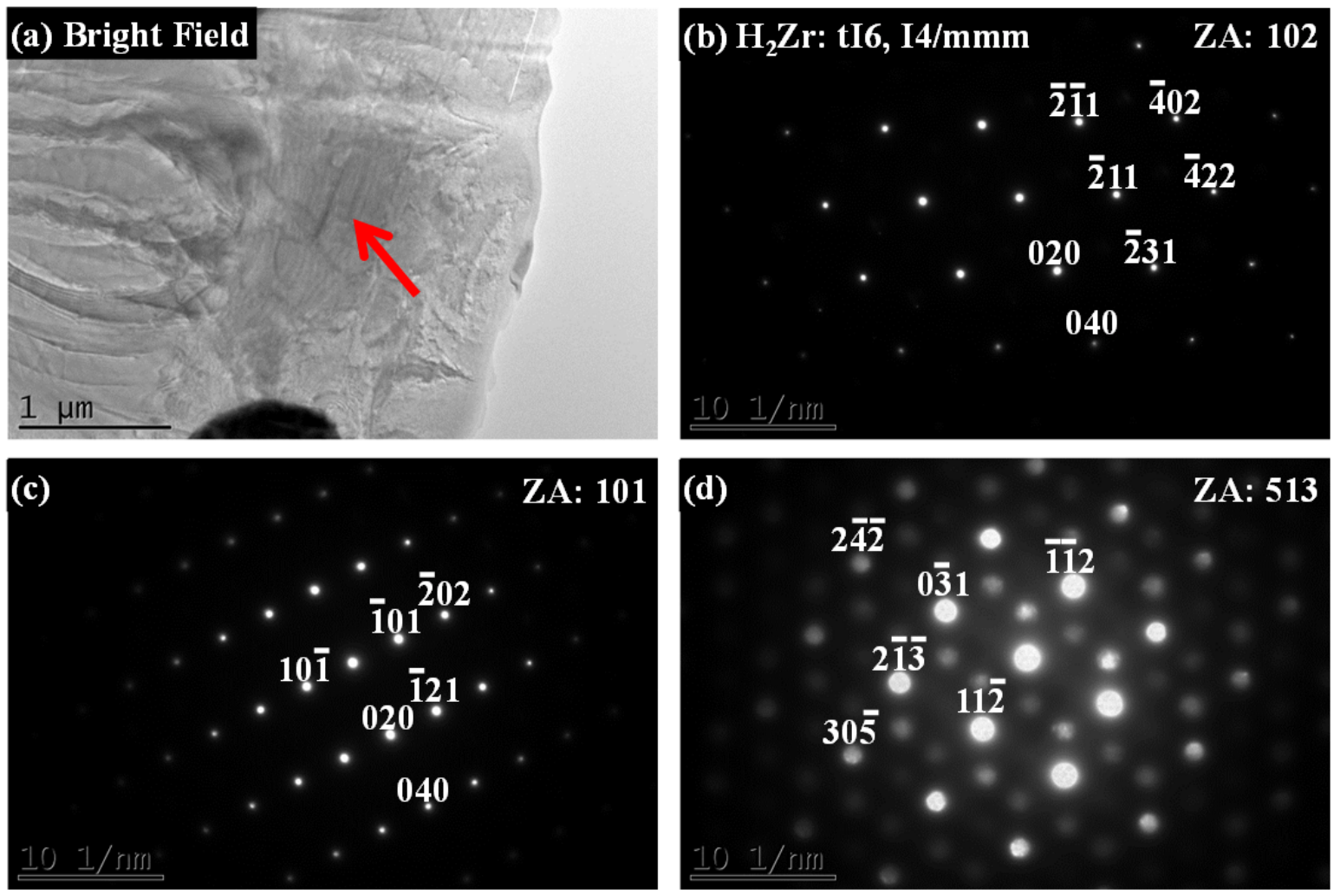

Figure 19. (a) A BF micrograph of a selected grain (marked by a red arrow) from the Zr-H matrix. (b), (c), and (d) are the corresponding electron diffraction spot patterns, indexed as the tetragonal- $\mathrm{H}_{2} \mathrm{Zr}$ phase, displaying three different zone axes from the selected grain.

The BF micrograph in Figure 20(a) shows that the U-particles contain several grains, and shows a $\mathrm{U}$ grain (marked by a red arrow), within a U-particle, that was selected for analysis. The diffraction patterns in Figure 20(b) through Figure 20(d) show three different zone axes from the selected grain. The U-particles are composed of the orthorhombic $\alpha-\mathrm{U}(\mathrm{oS} 4, \mathrm{Cmcm})$ phase. Analysis of several particles (not shown) confirmed the presence of this phase. 

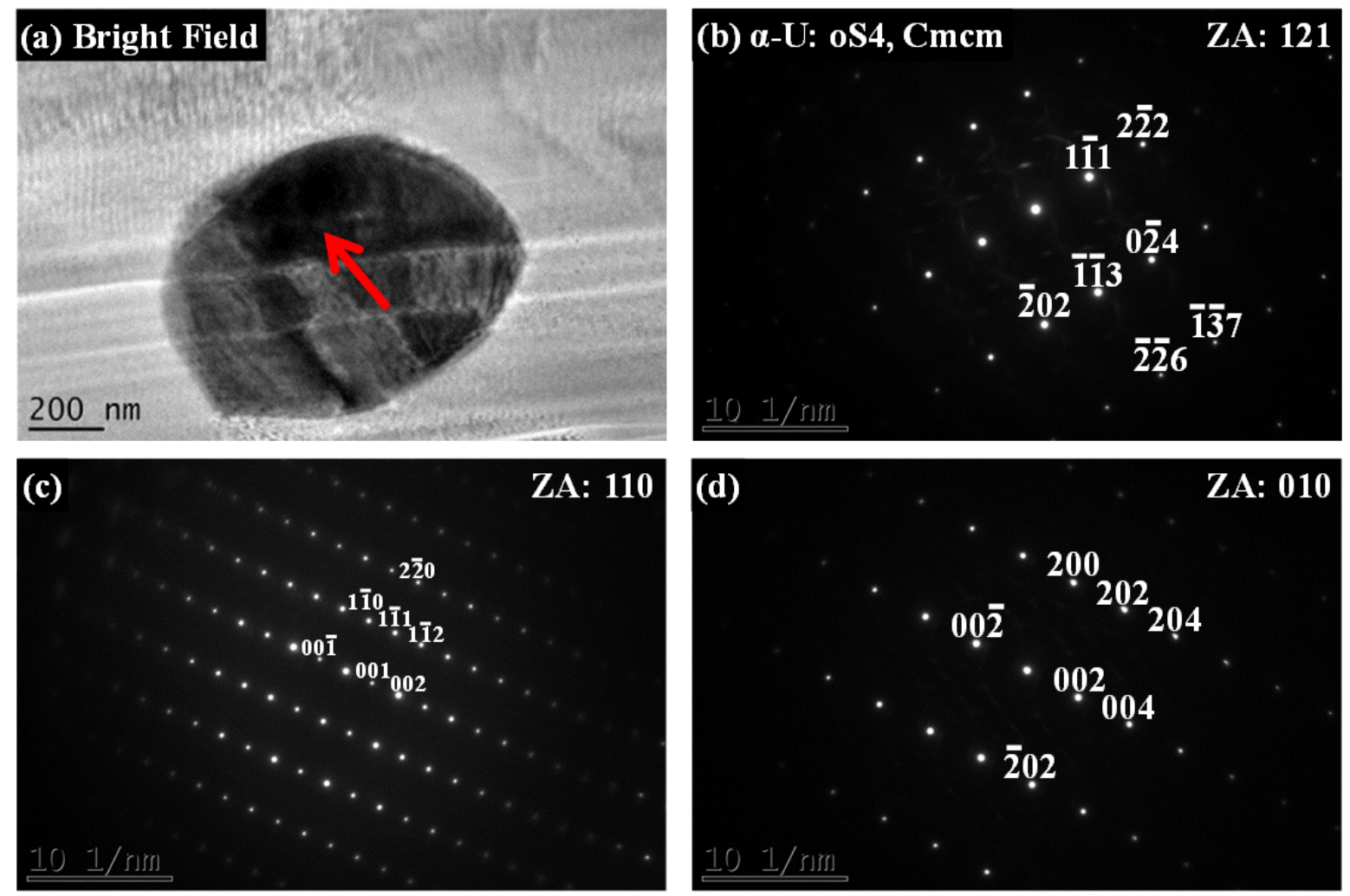

Figure 20. (a) A BF micrograph of a selected grain (marked by a red arrow) from a U-particle. (b), (c), and (d) show the corresponding diffraction patterns, indexed as the orthorhombic $\alpha-U$ phase, displaying three different zone axes from the selected grain.

Figure 21 shows (a) a BF micrograph of a grain of the Er-O phase selected within the fuel matrix grain boundaries. The diffraction patterns in Figure 21(b) through Figure 21(d) show three different zone axes from the selected grain. Diffraction analysis showed that the grains are composed of the $\mathrm{Er}_{2} \mathrm{O}_{3}$ (cI80, Ia-3) phase. All of the analyzed Er-O grains (not shown) contained the same crystal structure. 

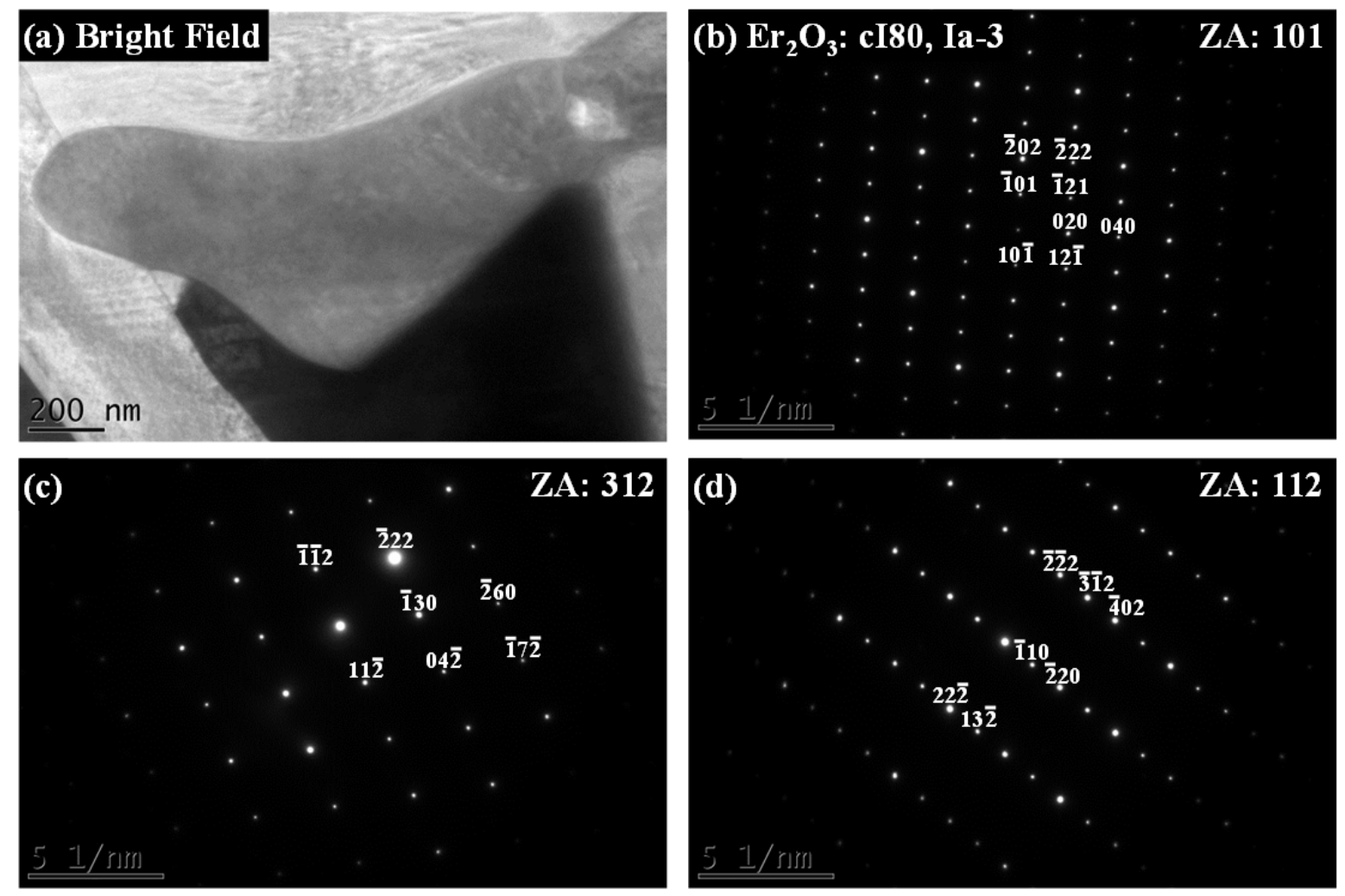

Figure 21. (a) A BF micrograph of a selected grain (marked by a red arrow) from an Er-O. (b), (c), and (d) show the corresponding diffraction patterns, indexed as the cubic- $\mathrm{Er}_{2} \mathrm{O}_{3}$ phase, displaying three different zone axes from the selected grain.

Diffraction data has been collected in the regions shown in Figure 15. The center of the BF micrographs in Figure 22(a) shows a lamella selected for analysis from this region. Figure 22(b) though (d) show the corresponding diffraction patterns. These patterns do not appear to fit the tetragonal $\mathrm{H}_{2} \mathrm{Zr}$ or cubic- $\mathrm{H}_{1.6} \mathrm{Zr}$ phases expected in the fuel matrix. These and other collected patterns, as of this writing, are under analysis. Diffraction data was also collected from the C-U-Zr interaction regions as shown for example in Figure 23. The diffraction patterns, as of the writing of this document, are under analysis to determine if the apparent interaction region is the result of chemical interactions or overlapping grains. The patterns in the C-U-Zr interaction regions where diffraction data was collected; however, at the time of this writing, the patterns do not appear to fit any $\mathrm{U}, \mathrm{Zr}, \mathrm{U}-\mathrm{Zr}$, or C-U-Zr phase for which crystallographic data was available. Diffraction data were not collected for the Er-O-U and Er-O-Zr interaction regions and remain a source for future analysis. The diffraction patterns in Figure 22 and Figure 23 are included in this report for reference. 

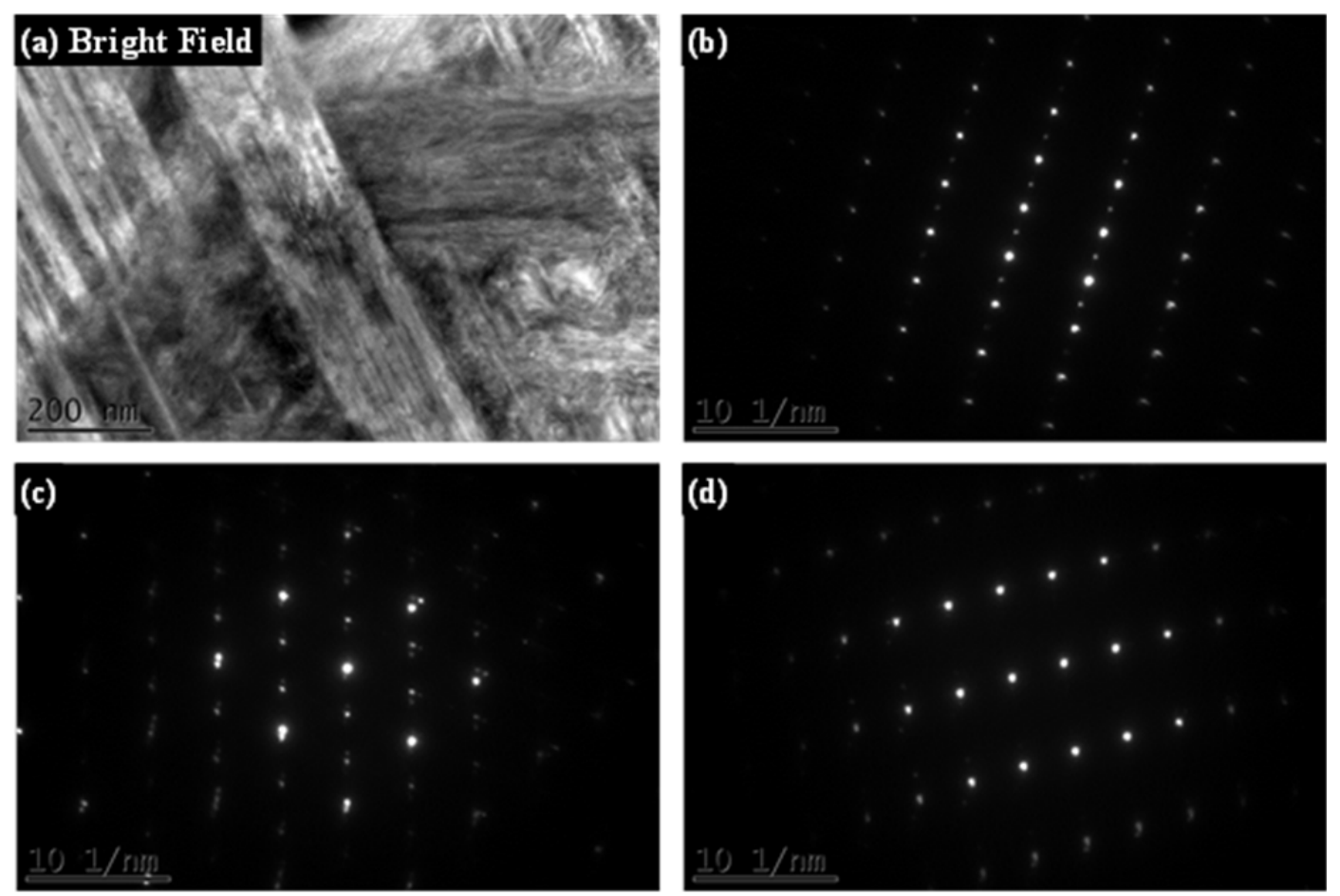

Figure 22. (a) Bright field micrograph of a selected from a lamella within a region similar to that shown in Figure 15. (b), (c), and (d) show the corresponding unindexed diffraction spot patterns displaying three different zone axes from the selected grain.
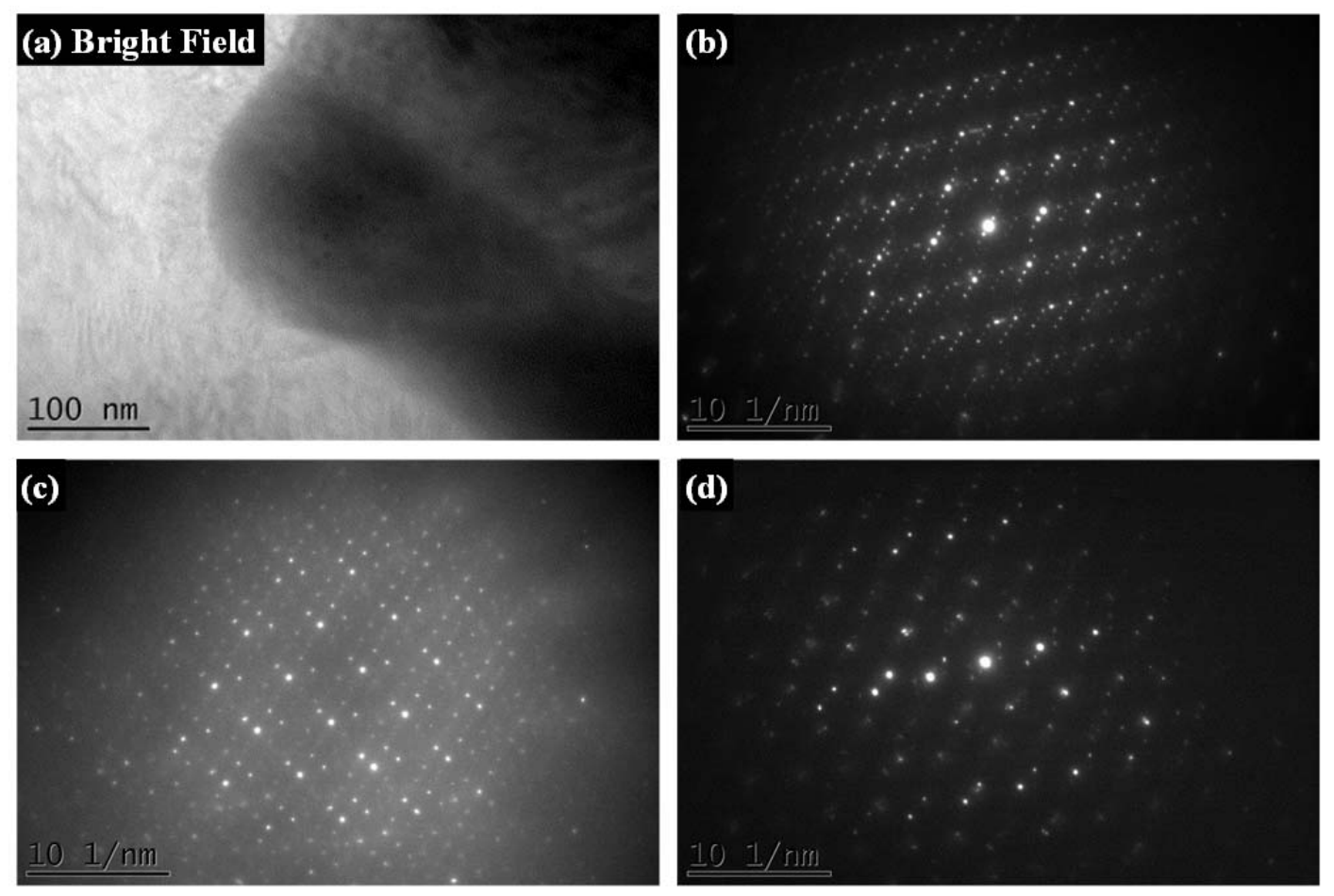

Figure 23. (a) Bright field micrograph of a selected U-particle/Zr-H-matrix interaction region. (b), (c), and (d) show the corresponding unindexed diffraction spot patterns displaying three different zone axes from the selected grain. 


\subsubsection{Analyses of Fine Features and Extra Reflections in Selected Diffraction Patterns}

Many of the electron diffraction patterns from the as-received samples showed streaks or reflections that could not be explained by diffraction from single crystals of any of the phases expected in the samples $\left(\mathrm{ZrH}_{2}, \mathrm{ZrH}_{1.6}, \mathrm{UZr}_{2}\right.$, or a body-centered cubic solid solution phase). Attempts were made to identify possible crystallographic or microstructural causes for these features by comparing images and diffraction patterns from selected areas. However, it must be emphasized that all interpretations presented here are preliminary hypotheses, which can only be tested by rigorous analysis and crystal-structure modeling. The time required for these analyses was beyond that available for this project. Below are selected grains of the fuel that may provide some insight on the possible causes of the extra reflections in the diffraction patterns from this study.

\subsubsection{Zr-H Matrix}

\section{Selected Grain 1}

Grain 1 shows two perpendicular sets of closely spaced, approximately periodic, planar features. One set of features appears to be oriented edge-on to the beam in Zone 1, and produces sharp streaks in the diffraction patterns (Figure 24). This diffraction pattern has reflections corresponding to a d-spacing larger than that of any commonly documented $\mathrm{Zr}$ hydride, suggesting possible formation of a two-layer superlattice.

Streaks are less pronounced or absent in other zone axes, where images suggest that the planar features are less precisely edge-on to the beam. It seems plausible (although not certain) that both sets of planar features are capable of producing conspicuous streaks and possible superlattices.

Figure 24 shows an image and the central part of the corresponding rotation-corrected diffraction pattern. The diffraction pattern shows rows of alternating bright and fainter spots. The d-spacing corresponding to the faint spots is $\sim 5.65 \AA$, which is too large to be produced by any of the Zr hydride phases in the International Centre for Diffraction Data's PDF4+ 2014 database. If it is assumed that the bright reflections are from the primary crystal lattice, the faint reflections may be from a superlattice formed by stacking two similar layers of material, each about $2.82 \AA$ thick.

The diffraction pattern also shows streaks between the reflections in each row (e.g., at arrows). The orientation of these reflections is consistent with diffraction from planar features in the orientation shown by the solid white lines in the image. Streaks like these are commonly caused by variations in stacking order in layered structures.

In combination, the image and diffraction pattern suggest that the material has a layered structure made of parallel units $\sim 2.82 \AA$ thick, which are stacked to form a superlattice with a period of $\sim 5.65 \AA$. The layers are parallel to the white lines drawn on the image, and are edge-on to the electron beam.

The image in Figure 24 also shows a second set of linear features whose orientation is shown by the dotted line. These features do not appear to be edge-on to the beam. They may be responsible for the elongation of reflections like the one in the upper-right corner of the diffraction pattern, but do not cause superlattice reflections or conspicuous streaks. 

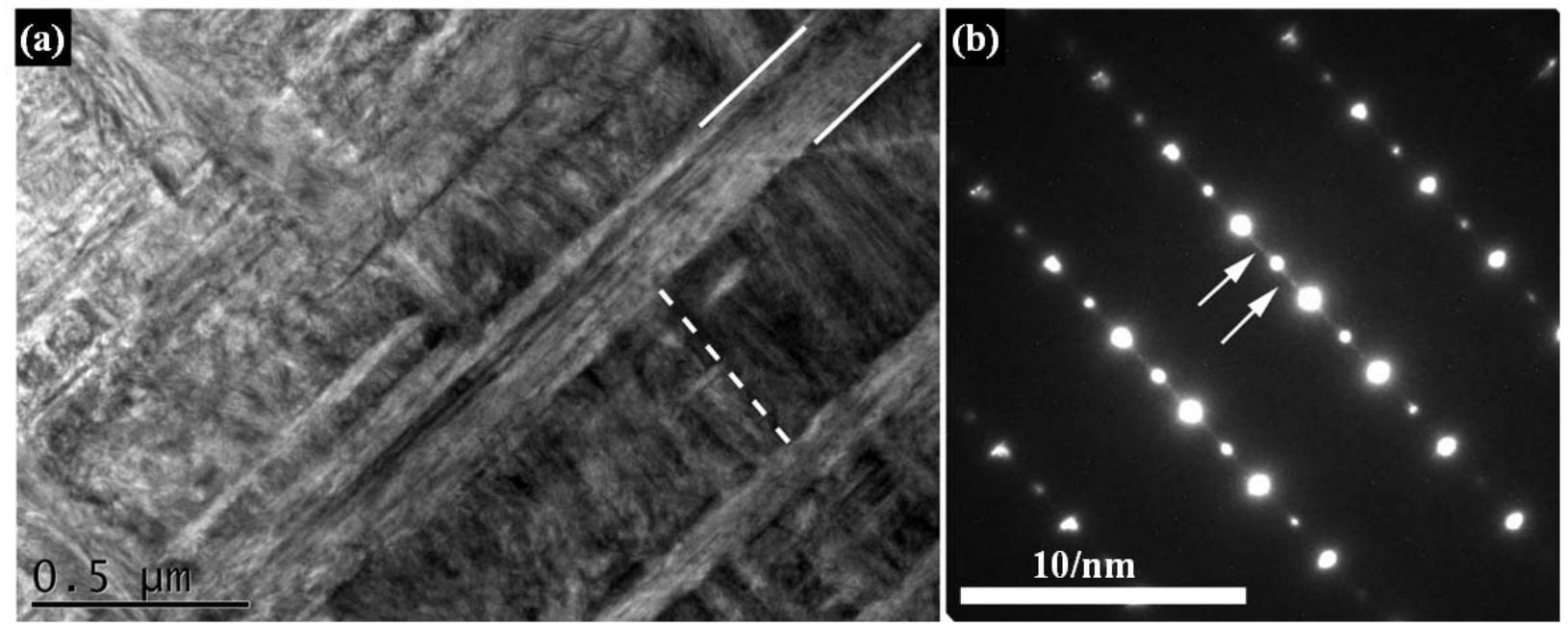

Figure 24. Selected Zr-H grain (grain 1, zone 1) in the matrix. (a) Bright field micrograph of the selected area with solid and dashed lines showing orientations of two sets of linear features. (b) Central part of diffraction pattern. Arrows show streaks whose orientation is consistent with diffraction from features parallel to the solid lines in the image.

Figure 25 shows the same area, viewed from a different direction. The image shows two sets of parallel features, neither of which appears to be edge-on to the beam. The diffraction pattern has faint streaks whose orientation is consistent with diffraction from features parallel to the solid line in the image. Variations in the intensity of the reflections in the middle of the streaks (e.g., at large arrows) suggest that they may be from another crystal.

Figure 26 shows the same area, viewed from a third direction. The image shows two sets of parallel features, both of which appear to be almost edge-on to the beam. The diffraction pattern has faint streaks whose orientation is consistent with diffraction from both sets of features.
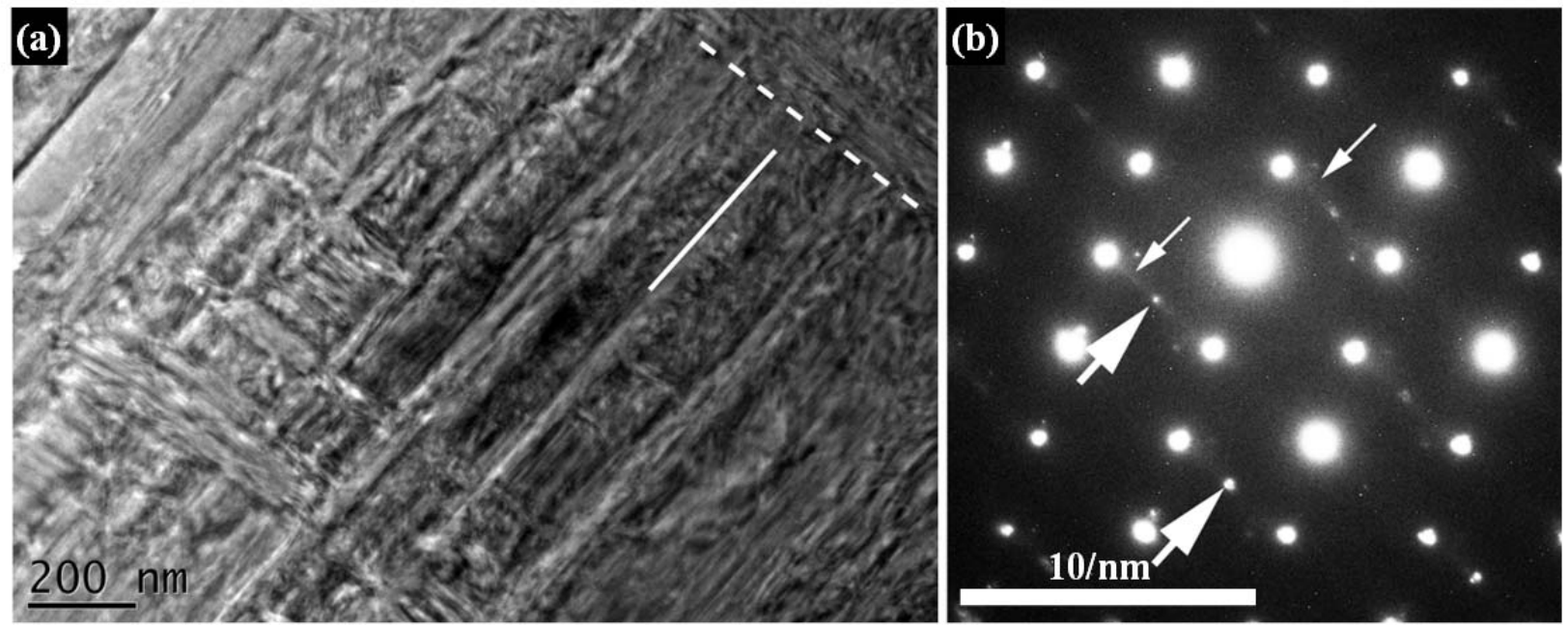

Figure 25. Selected Zr-H grain (grain 1, zone 2) in the matrix. (a) Bright field micrograph of the selected area where solid and dashed lines show orientations of two sets of linear features. (b) Central part of diffraction pattern. Small arrows show streaks whose orientation is consistent with diffraction from features parallel to the solid lines in the image. Large arrows show examples of reflections in the centers of the streaks. 

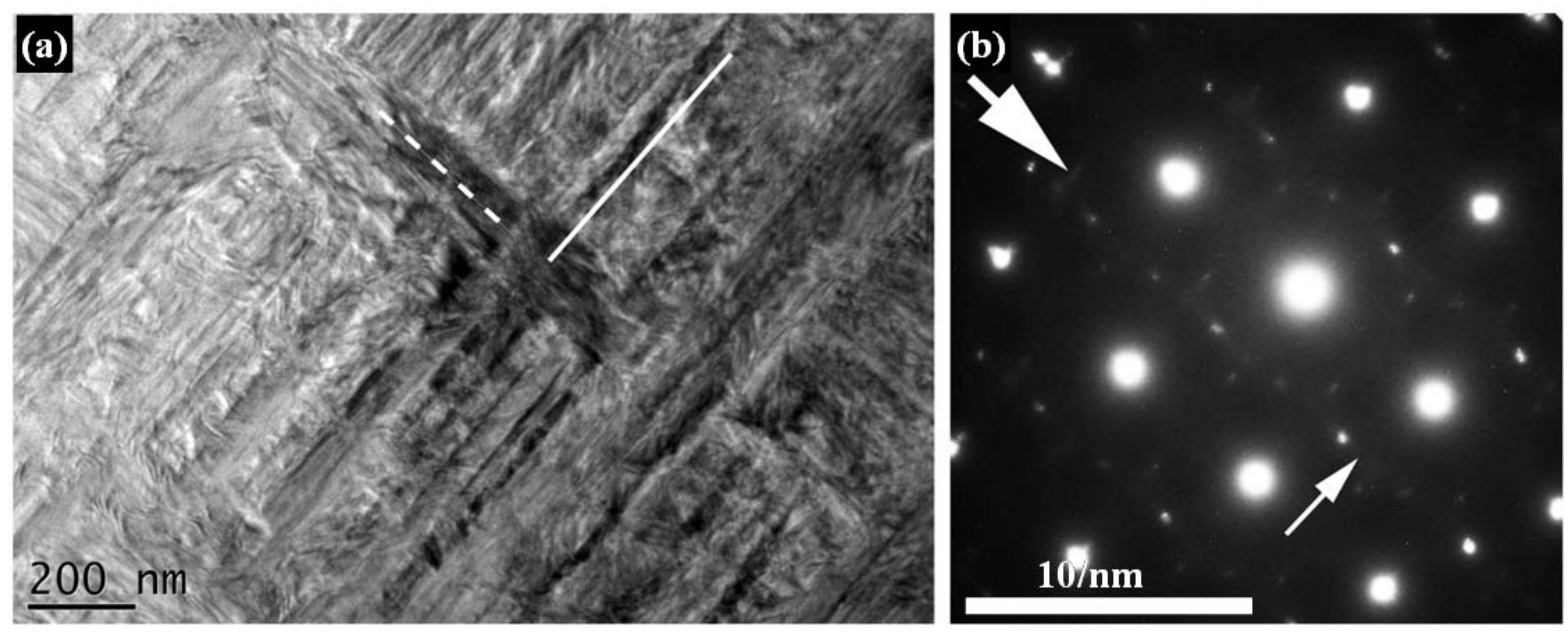

Figure 26. Selected Zr-H grain (Grain 1, zone 3) in the matrix. (a) Bright field micrograph of the selected area where solid and dashed lines show orientations of two sets of linear features. (b) Central part of diffraction pattern. Small arrow shows an example of a faint streak whose orientation is consistent with diffraction from features parallel to the solid lines in the image. Large arrow shows an example of a faint streak whose orientation is consistent with diffraction from features parallel to the dotted lines in the image.

\section{Selected Grain 2}

Figure 27 shows an image and part of the corresponding rotation-corrected diffraction pattern. The image shows closely spaced planar features in some areas. The orientation of these features changes from place to place, possibly as a result of deformation in this area of the sample.

The only diffraction pattern from this grain that was analyzed shows rows of bright reflections with three equally spaced fainter reflections between them. The faint reflections correspond to a d-spacing of $\sim 8.2 \AA$ (much larger than would be produced by any of the Zr hydrides in the ICDD PDF4+ 2014 database). Some of the faint reflections are elongated perpendicular to the linear features in the image, suggesting that the lattice may be strained in this direction. Variations in intensity of the fainter reflections suggest that they are not from a 4-repeat superlattice. These reflections are probably formed by multiple scattering events, possibly involving crystals with similar characteristics superimposed on one another. 

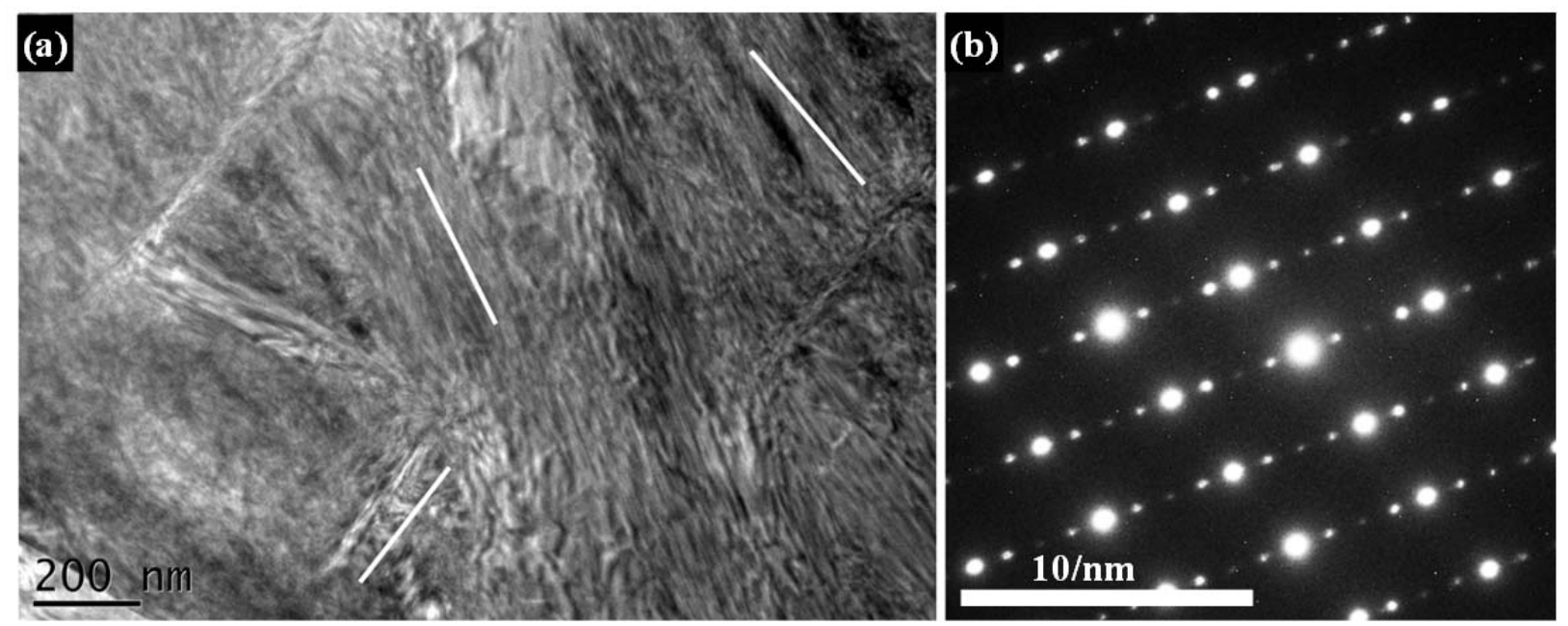

Figure 27. Selected Zr-H grain (Grain 2, zone 3) in the matrix. (a) Bright field micrograph of the selected area where white lines show orientations of linear features in different areas. (b) Central part of diffraction pattern.

\section{Selected Grain 3}

Images of this material are too low magnification to show details comparable to those in other images. However, the images suggest that the material has at least one direction of closely spaced planar features. Diffraction patterns have two sets of very faint, elongated reflections. The origins of the faint reflections cannot be conclusively determined without extensive research; however, one possibility is multiple diffraction involving two phases with similar structures and crystal orientations but slightly different lattice parameters.
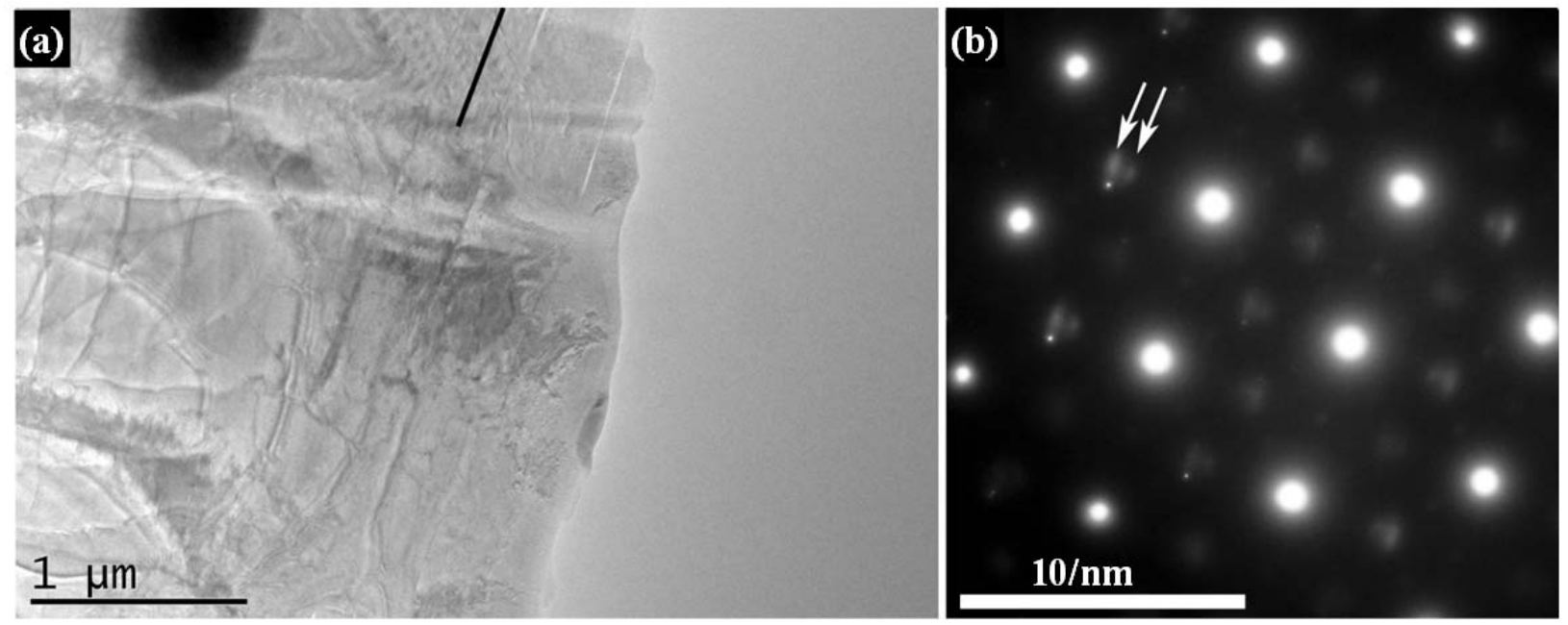

Figure 28. Selected Zr-H grain (Grain 3, Zone 1) in the matrix. (a) Bright field micrograph of the selected area with black line showing orientation of closely spaced linear features. (b) Corresponding diffraction pattern, with arrows showing example of faint paired reflections.

Figure 28 shows an image and part of the corresponding rotation-corrected diffraction pattern. The image shows numerous closely spaced features parallel to the black line. 
The diffraction pattern shows numerous pairs of faint, elongated reflections with diffuse edges. The long direction of the reflections is parallel to the linear features in the image, indicating that the reflections do not represent diffraction from the boundaries of the linear features. One possible explanation for these reflections is double diffraction involving three phases: one phase producing the bright reflections and two phases with similar structures and orientations but slightly different lattice parameters producing the paired reflections. However, further detailed investigation is required to understand these reflections.
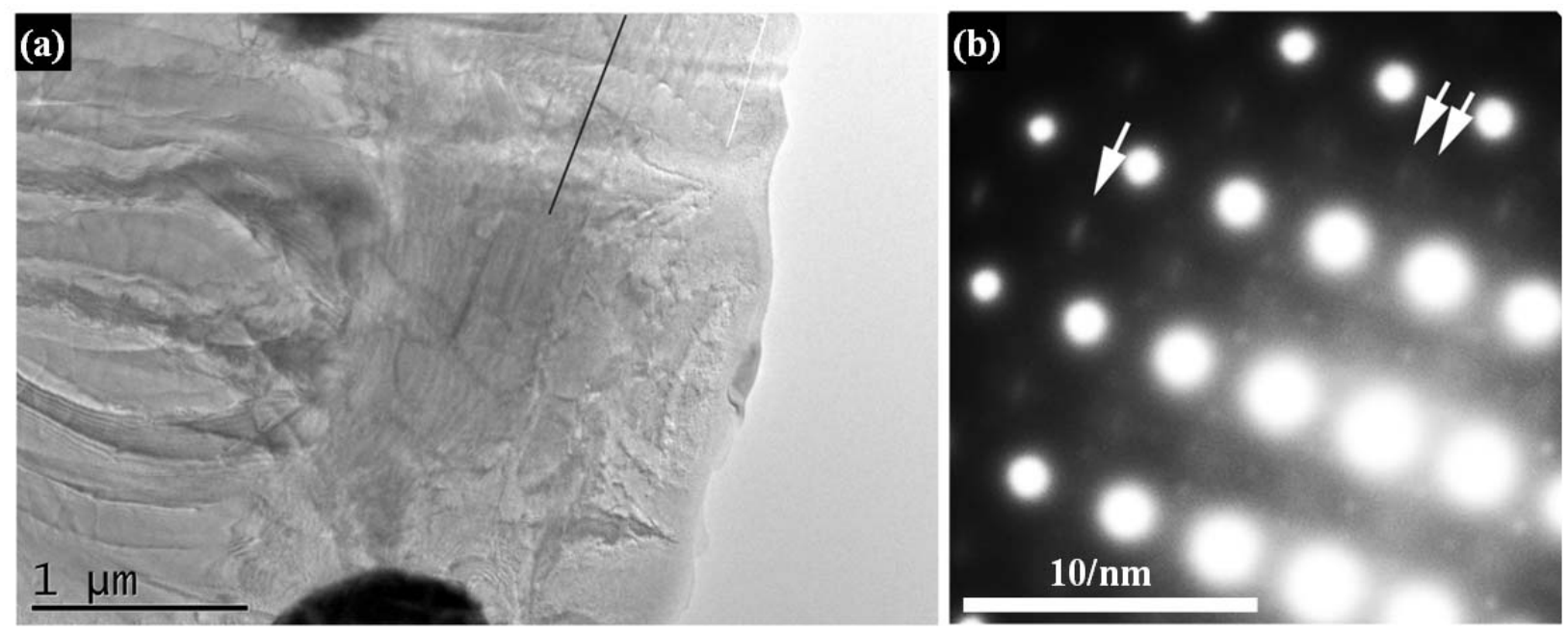

Figure 29. Selected Zr-H grain (Grain 3, zone 2) in the matrix. (a) Bright field micrograph of the selected area with black line showing orientation of closely spaced linear features. (b) Corresponding diffraction pattern, with arrows showing examples of very faint elongated reflections.

Figure 29 shows the same area of the sample from a different orientation. The closely spaced linear features are more visible in this image than in Figure 28, and the elongated reflections in the diffraction pattern are fainter. The reflections probably have the same origin as those in Figure 28; however, further detailed investigation is required to understand what causes them.

\subsubsection{U-Zr Phases}

\section{Selected Grain 4}

This is the only grain examined in detail from an area where the Energy-Dispersive X-ray spectrum shows both $\mathrm{U}$ and $\mathrm{Zr}$. Images show a regular arrangement of dark spots that apparently does not correspond to recognizable features in the diffraction pattern. Diffraction patterns show relatively widely spaced bright reflections and numerous less-intense extra reflections. Some of the extra reflections are in closely spaced lines, and may indicate multiple diffractions from more than one crystal. Other extra reflections form patterns similar to the bright reflections, but offset by consistent amounts. Although details differ between diffraction patterns, the extra reflections probably all represent diffraction from more than one crystal.

Figure 30 shows data from an area whose Energy-Dispersive X-ray spectrum shows both $\mathrm{U}$ and $\mathrm{Zr}$. The images show an area of high-Zr matrix (light contrast) at the left, and at least two crystals of U-bearing material at right. Small arrows in Figure 30(a) show examples of dark spots in a regular arrangement. The orientations of rows of dark spots do not correspond to any obvious features in diffraction patterns, suggesting that the dark spots may be interference phenomena caused by overlapping crystal lattices. 
Figure 30(c) is a diffraction pattern that shows a pattern with relatively widely spaced bright reflections, each of which is part of a line of closely spaced reflections. The d-spacing corresponding to the distance between the closely spaced reflections is $\sim 15.6 \AA$, which is larger than can be produced by any known U--Zr phase. These reflections probably result from overlapping crystal lattices. Figure 30c also shows streaks, suggesting that the material contains a layered structure not obviously visible in the image.

Figure 30(c) is a diffraction pattern from the same area but a different zone axis. It shows a regular pattern of widely spaced bright reflections and numerous less intense reflections whose spacing and orientation are the same as those of the bright reflections. This pattern of reflections could be explained by double diffraction (i.e., some of the electrons diffracted in the direction of the bright reflections undergo a second scattering event to produce the faint reflections) or by single scattering events from multiple crystals with similar structures and different orientations.

Taken together, the images and diffraction patterns strongly suggest that data from this area is not from a single crystal, but instead represents overlapping crystals. The possibility that one of these crystals is a high- $\mathrm{U}$ phase and the other is part of the high-Zr matrix should be considered.

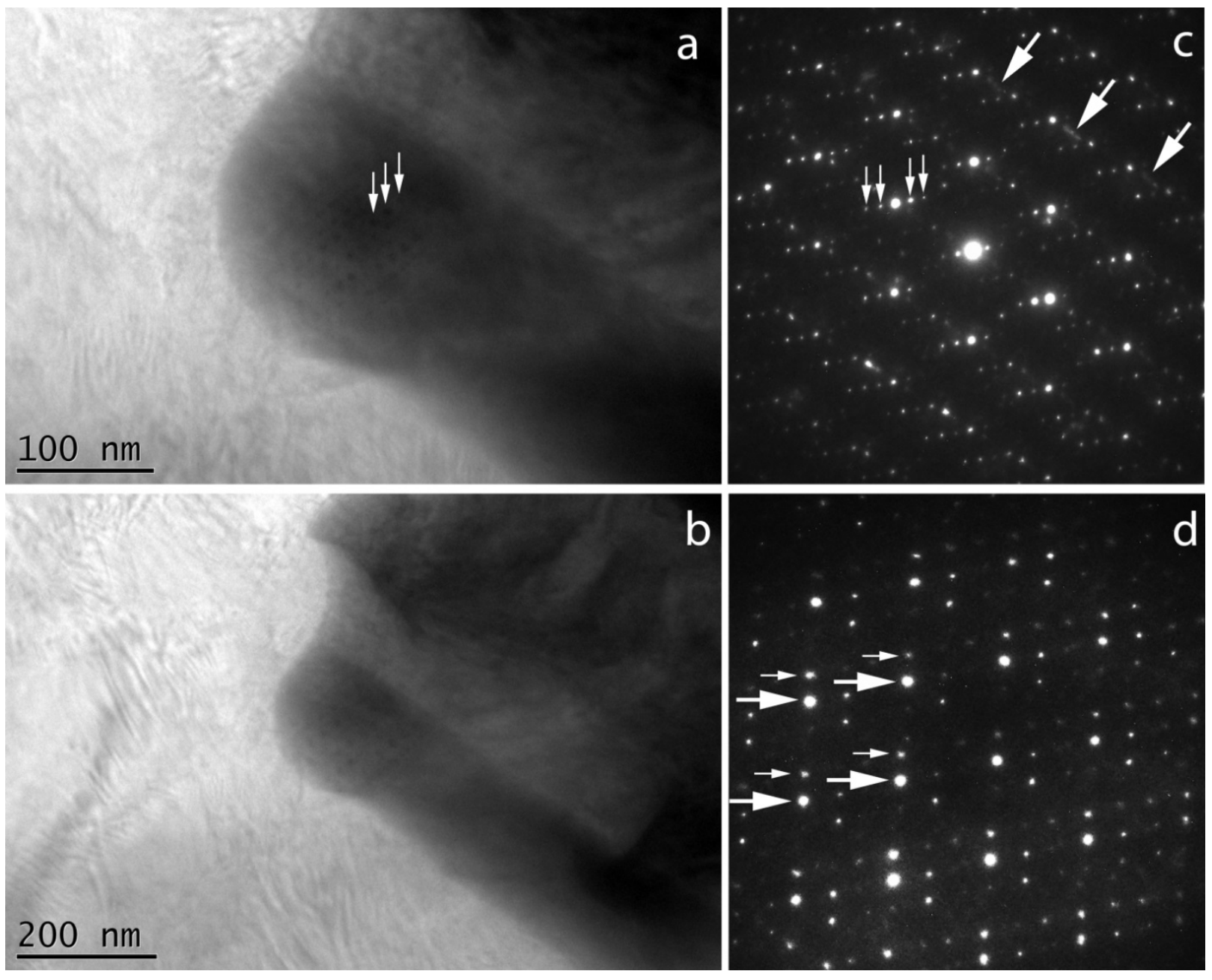

Figure 30. Selected U-Zr grain (Grain 3, Zone 2). (a) and (b) Bright field micrograph of the selected areas at different magnifications. Arrows in (a) show examples of dark spots in a regular pattern. (c) Diffraction pattern, with small arrows showing a line of faint reflections and large arrows showing streaks between reflections. (d) Diffraction pattern, with faint reflections (e.g., at small arrows) forming a pattern similar to bright reflections (large arrows) but offset by a consistent distance and direction. 
3.1.3.3 Discussion and Conclusions. The diffraction patterns examined here show streaks or extra reflections. Although detailed explanations of these features suggest a variety of origins, the streaks and extra reflections appear to involve diffraction from multiple crystals or fine-scale layered structures.

Consideration of U-Zr, Zr-H, and U-Zr-H phase diagrams (see Sheldon and Peterson (1989) and La Grange et al.'s articles [6,7]) suggest that many materials containing U, Zr, and H originally crystallize as a U-Zr-H solid solution with a body-centered cubic structure, then separate into lower-temperature phases by solid-state reactions during cooling. Transformations in U-Zr systems are known to produce fine intergrowths of multiple crystals, leading to diffraction patterns with numerous "extra" reflections, even when only one phase is present [8]. It seems plausible that similar processes might occur during evolution of microstructures in ternary $\mathrm{U}-\mathrm{Zr}-\mathrm{H}$ phases. If so, microstructures and associated features in diffraction patterns are likely to be strongly influenced by the precise composition and the thermal and processing histories of individual samples and analysis areas.

It is not possible to determine from the present data whether the fine-scale layered features in the high-Zr matrix involve crystals of one phase in multiple orientations, or whether they may be intergrowths of two or more phases on a scale of a few Angstroms. In either case, a far larger fraction of the atoms are close to grain or phase boundaries than would occur in single crystals with similar volumes. Minimization of excess free energy from these interfaces may lead to displacements of atoms close to the interfaces, causing distortion of crystal structures and complicating phase identifications.

These analyses have suggested numerous possible explanations for streaks and "extra" reflections in diffraction patterns. It must be emphasized that these are only suggestions, which must be tested by detailed analyses of crystal structures before they are accepted as conclusive. This testing is likely to involve crystal-structure modeling and simulation of high-resolution TEM images, and is far beyond the scope of typical fuels characterization projects.

\subsection{Characterization of TRIGA Fuel Annealed at $950^{\circ} \mathrm{C}$}

TRIGA fuel samples were annealed at $950^{\circ} \mathrm{C}$ for 5,16 , and 24 hours to study the effect of high temperature on the fuel microstructure and the microstructural evolution of the fuel as a function of time. During annealing, two thermocouples were inserted into the furnace to monitor the furnace temperature and to generate a record of the furnace conditions as a function of time. Figure 31 shows the furnace temperature conditions as a function of time for the fuel samples annealed at $950^{\circ} \mathrm{C}$ for (a) 5 , (b) 16 , and (c) 24 hours. Note that two temperature profiles are shown in each graph: one colored magenta and the other blue. The profiles in each graph are nearly identical and on top of each other. The line thickness for the profile colored blue was thickened so that it was visible in the graph. The thermocouple data shows that the furnace experienced negligible temperature variations during annealing at the three specified annealing times. 

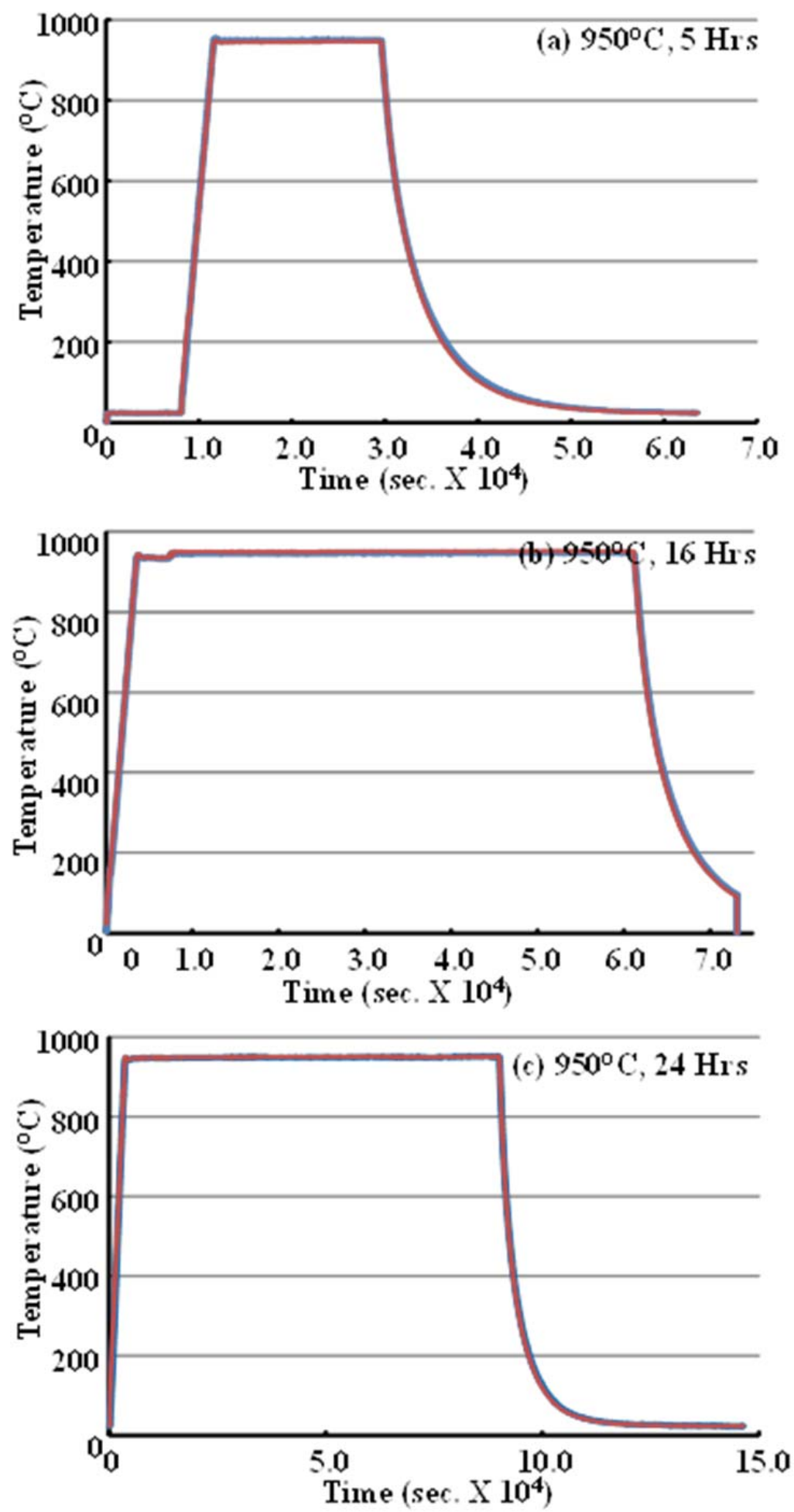

Figure 31. Temperature profiles generated from the TRIGA fuel annealing process at $950^{\circ} \mathrm{C}$ for (a) 5 , (b) 16, and (c) 24 hours. 


\subsubsection{Characterization by Scanning Electron Microscopy}

TRIGA fuel samples were annealed at $950^{\circ} \mathrm{C}$ for 5,16 , and 24 hours. Figure 32 through Figure 34 show low and higher magnification BE micrographs of the fuel after annealing for 5, 16, and 24 hours, respectively. The figures show the fuel matrix as a light-gray contrast phase. Dark-contrast phase regions are dispersed through the matrix. The higher detail micrographs in Figure 32(b), Figure 33(b), and Figure 34(b) show that a mid-gray contrast phase discontinuously surrounds the dark-contrast phase regions, suggesting the presence of an additional phase in the fuel microstructure. Another mid-gray phase with an apparent acicular microstructure is distributed throughout the matrix. The BE micrographs indicate that, after annealing, the fuel microstructure may contain at least three or four different phases. Comparison of Figure 32(a), Figure 33(a), and Figure 34(a) shows that the acicular phase appears to increase in volume fraction and coarsens with increased annealing time.

Comparison of these micrographs to those in Figure 7 and Figure 8 shows that the U-particles are not discernable after annealing, indicating that the particles have fully interacted with the $\mathrm{Zr}-\mathrm{H}$ matrix. The dark contrast features shown in Figure 7(b) appear to have endured the annealing process. The mid-gray phase surrounding the dark-gray phase in Figure 32(b), Figure 33(b), and Figure 34(b) may be the result of interactions between the matrix and dark-gray phase.
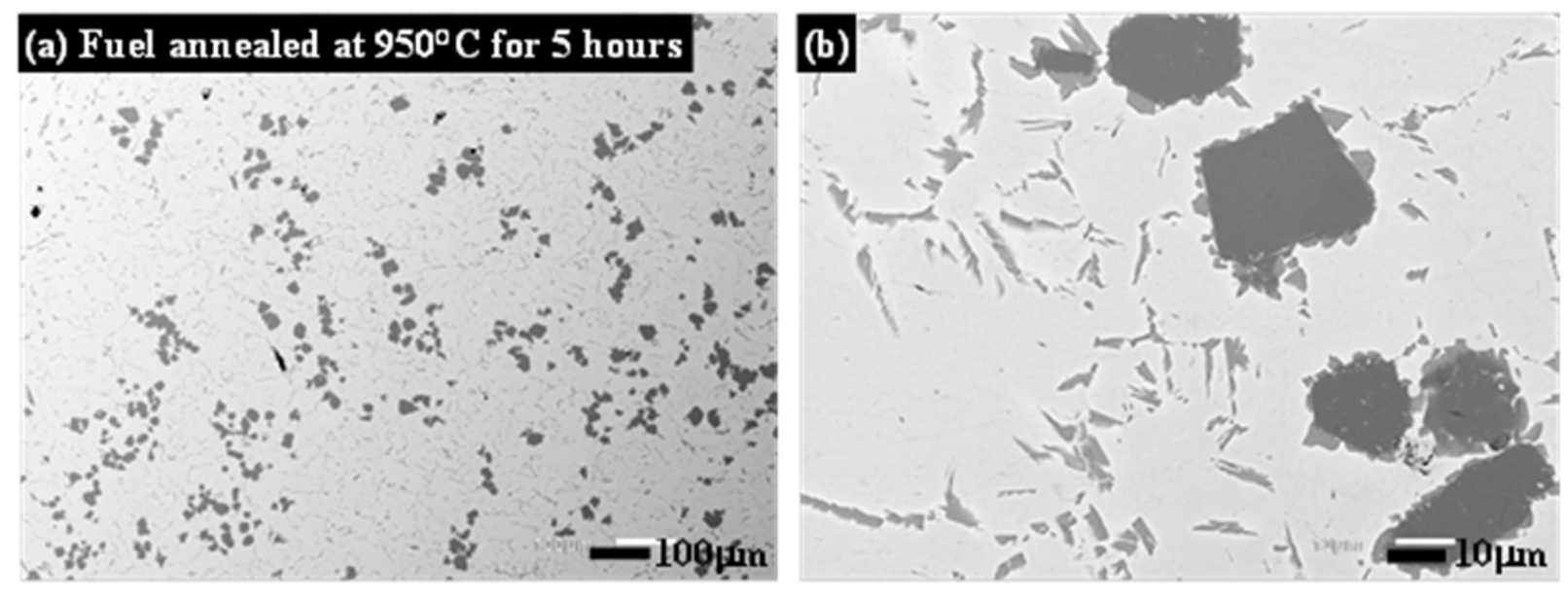

Figure 32. TRIGA fuel microstructure after annealing at $950^{\circ} \mathrm{C}$ for 5 hours: (a) low and (b) higher magnification BE micrographs.
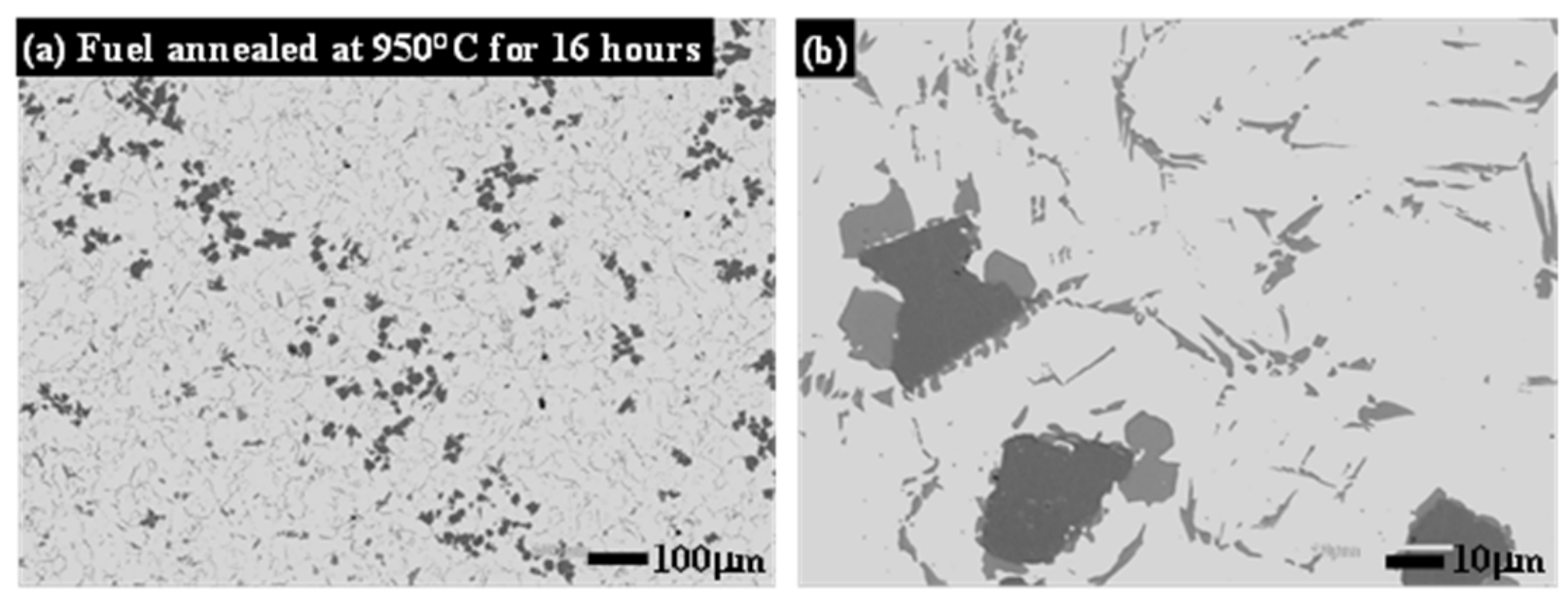

Figure 33. TRIGA fuel microstructure after annealing at $950^{\circ} \mathrm{C}$ for 16 hours: (a) low and (b) higher magnification BE micrographs. 

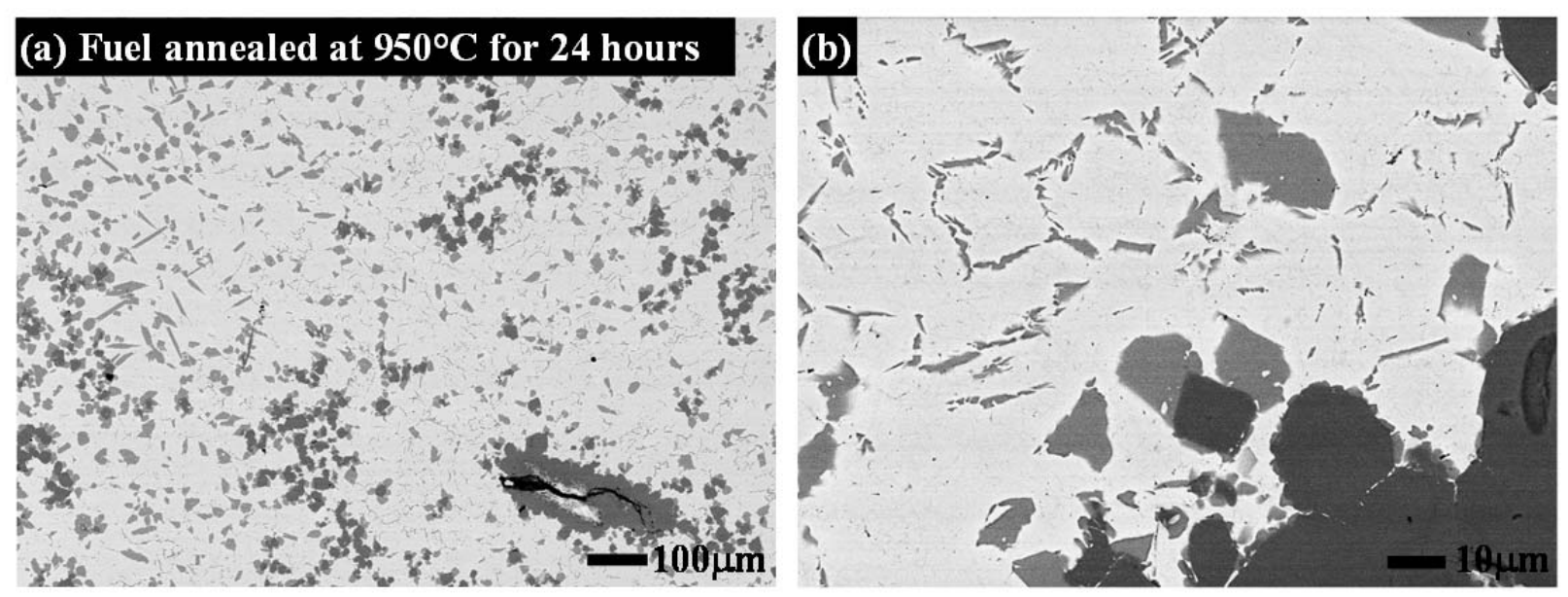

Figure 34. TRIGA fuel microstructure after annealing at $950^{\circ} \mathrm{C}$ for 24 hours: (a) low and (b) higher magnification BE micrographs.

X-ray maps were collected from selected regions of the samples to qualitatively identify the presence and location of $\mathrm{U}, \mathrm{Zr}$, and $\mathrm{Er}$ in the microstructures. Figure 35 through Figure 37 show the X-ray maps collected from the samples annealed for 5, 16, and 24 hours, respectively. The maps show that after annealing at $950^{\circ} \mathrm{C}$, the fuel matrix contains $\mathrm{U}$ and $\mathrm{Zr}$. This confirms that the U-particles, present prior to annealing, have fully interacted with the $\mathrm{Zr}-\mathrm{H}$ matrix. The dark-gray phase regions appear to contain only $\mathrm{Zr}$. The mid-gray regions also appear to contain only $\mathrm{Zr}$. These regions show slightly higher contrast in the $\mathrm{Zr}$ x-ray maps when compared to the dark-gray phase, suggesting a relatively higher $\mathrm{Zr}$ concentration in the mid-gray phase or phases. This observation may be indicative of a difference in hydrogen content between the dark- and mid-gray phase regions.

$\mathrm{Er}_{2} \mathrm{O}_{3}$ was found segregated at the grain boundaries in the as-received samples. The X-ray maps for $\mathrm{Er}$ in Figure 35(d), through Figure 37(d) indicate that the Er in the annealed samples is still segregated, likely at grain boundaries. The locations of Er in the microstructures of the three samples annealed at $950^{\circ} \mathrm{C}$ do not generally correspond with the locations of the acicular mid-gray phases. 

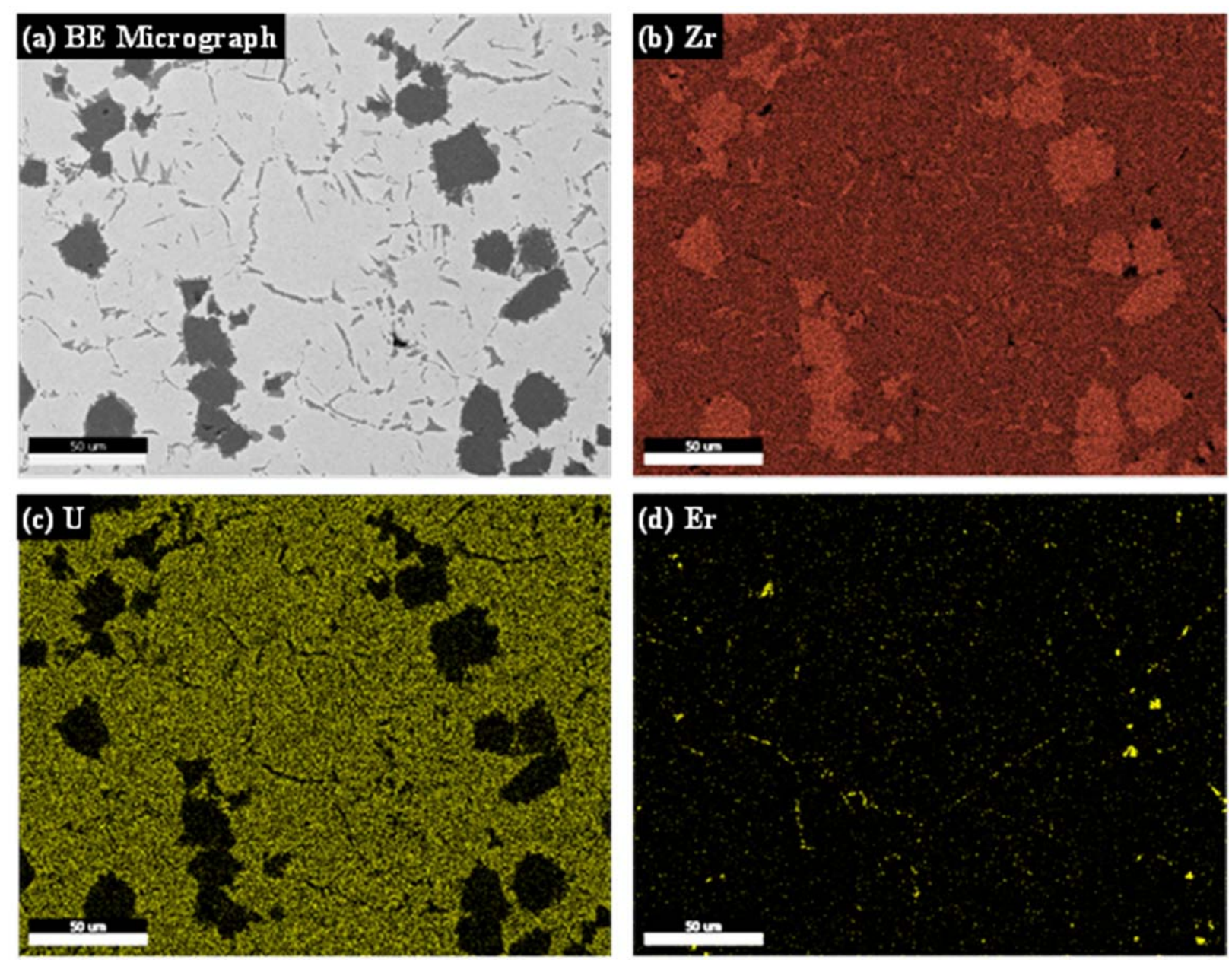

Figure 35. (a) A selected area of the TRIGA fuel annealed at $950^{\circ} \mathrm{C}$ for 5 hours. (b)-(d) Qualitative x-ray maps showing the presence and location of (b) $\mathrm{Zr}$, (c) U, and (d) Er in the fuel microstructure. 

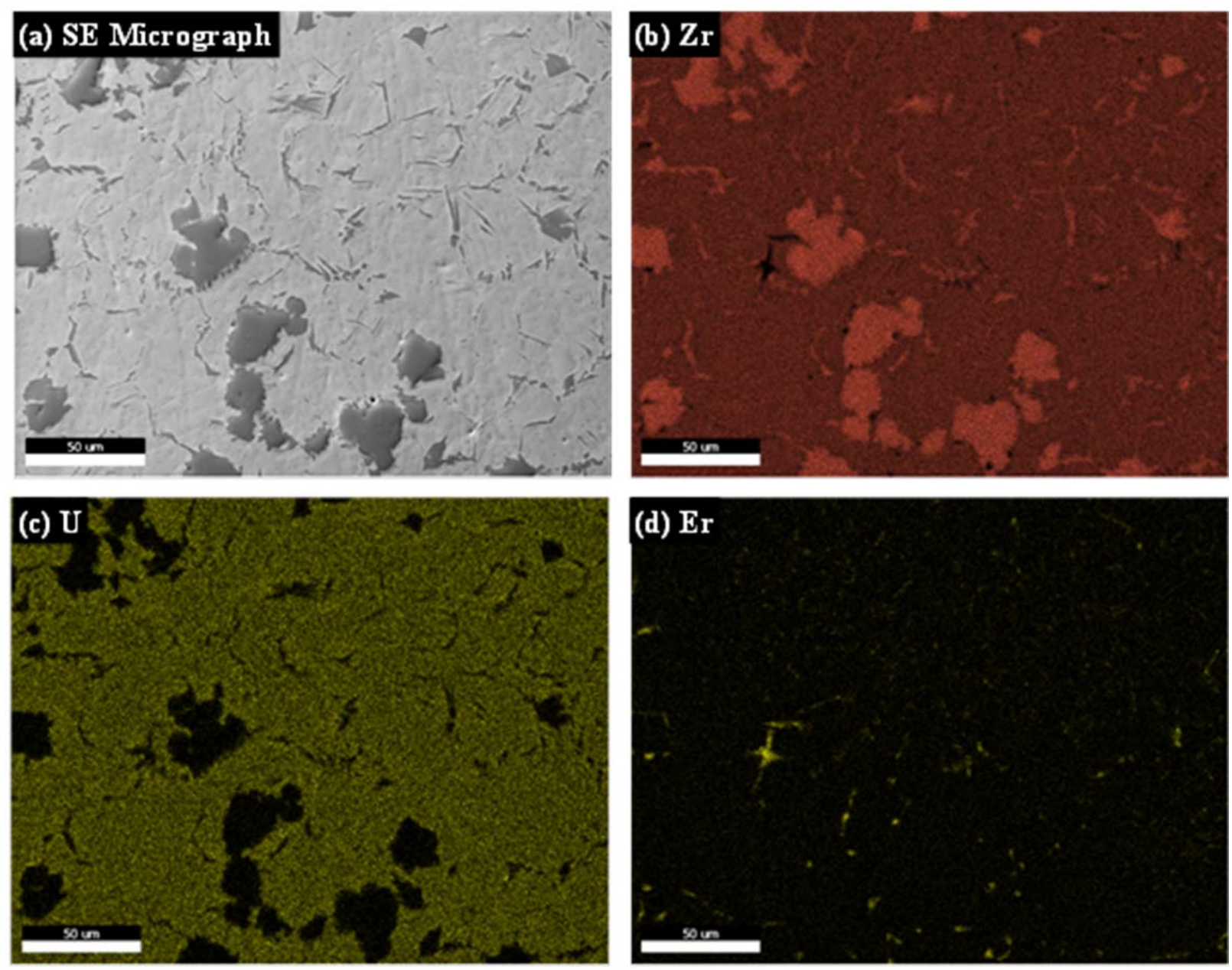

Figure 36. (a) A selected area of the TRIGA fuel annealed at $950^{\circ} \mathrm{C}$ for 16 hours. (b)-(d) Qualitative x-ray maps showing the presence and location of (b) $\mathrm{Zr}$, (c) U, and (d) Er in the fuel microstructure. 

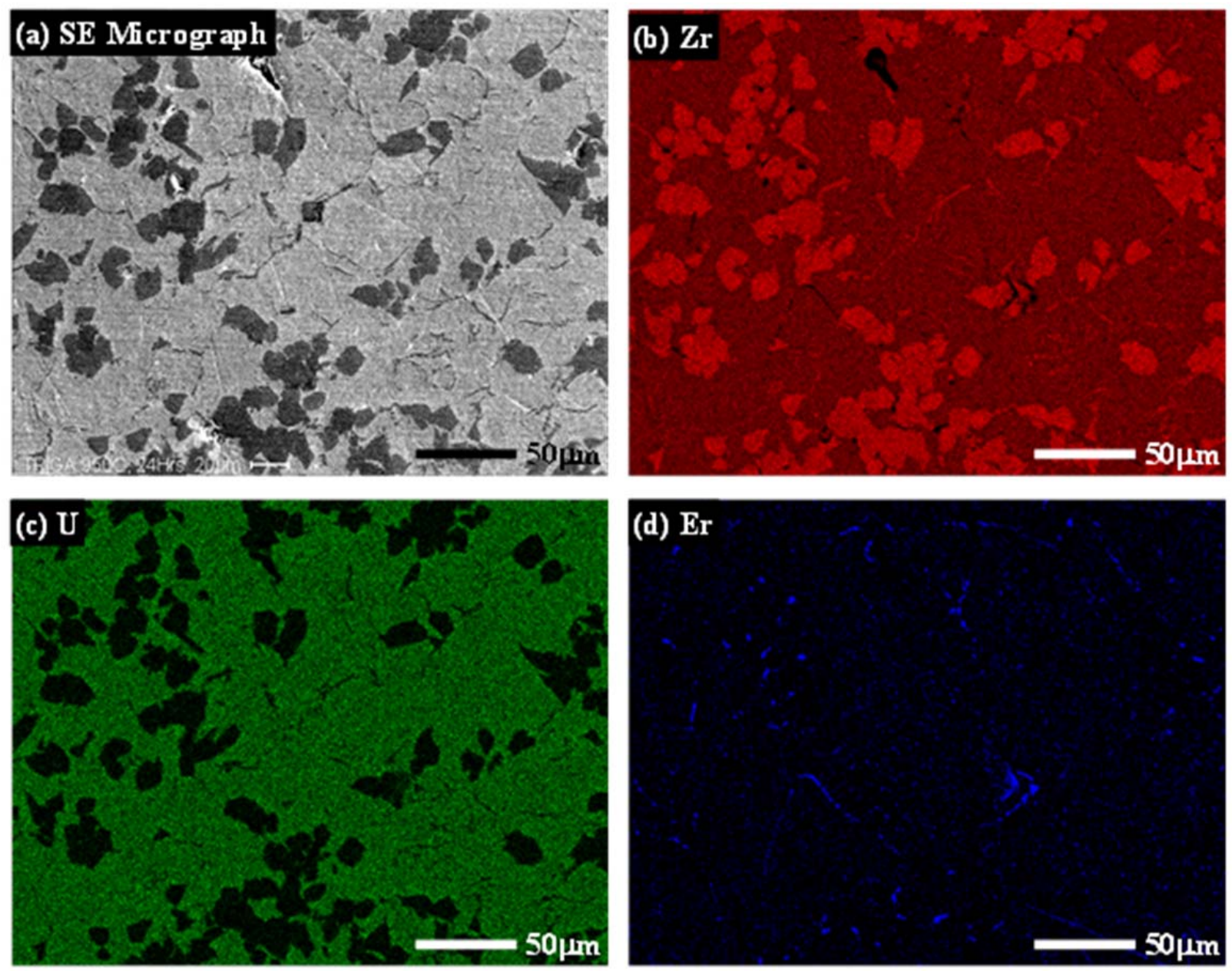

Figure 37. (a) A selected area of the TRIGA fuel annealed at $950^{\circ} \mathrm{C}$ for 5 hours. (b)-(d) Qualitative x-ray maps showing the presence and location of (b) $\mathrm{Zr}$, (c) U, and (d) Er in the fuel microstructure.

EDS analysis was performed on the different features in the microstructure of the fuel samples. Figure 38 through Figure 40 show EDS patterns from the samples annealed for 5, 16, and 24 hours, respectively. Table 1 through Table 3 show the results of standardless quantitative analysis. The analysis was performed based on phase contrast in the BE micrographs. The results show that after annealing the fuel matrix contains a significant concentration of $U$. The dark- and mid-gray phases contain negligible concentrations of $U$ and Er. Due to the interaction volume of the electron beam, EDS of the acicular features encompassed the acicular features and matrix, resulting in high error in the quantitative analysis. The data is included for completeness. 

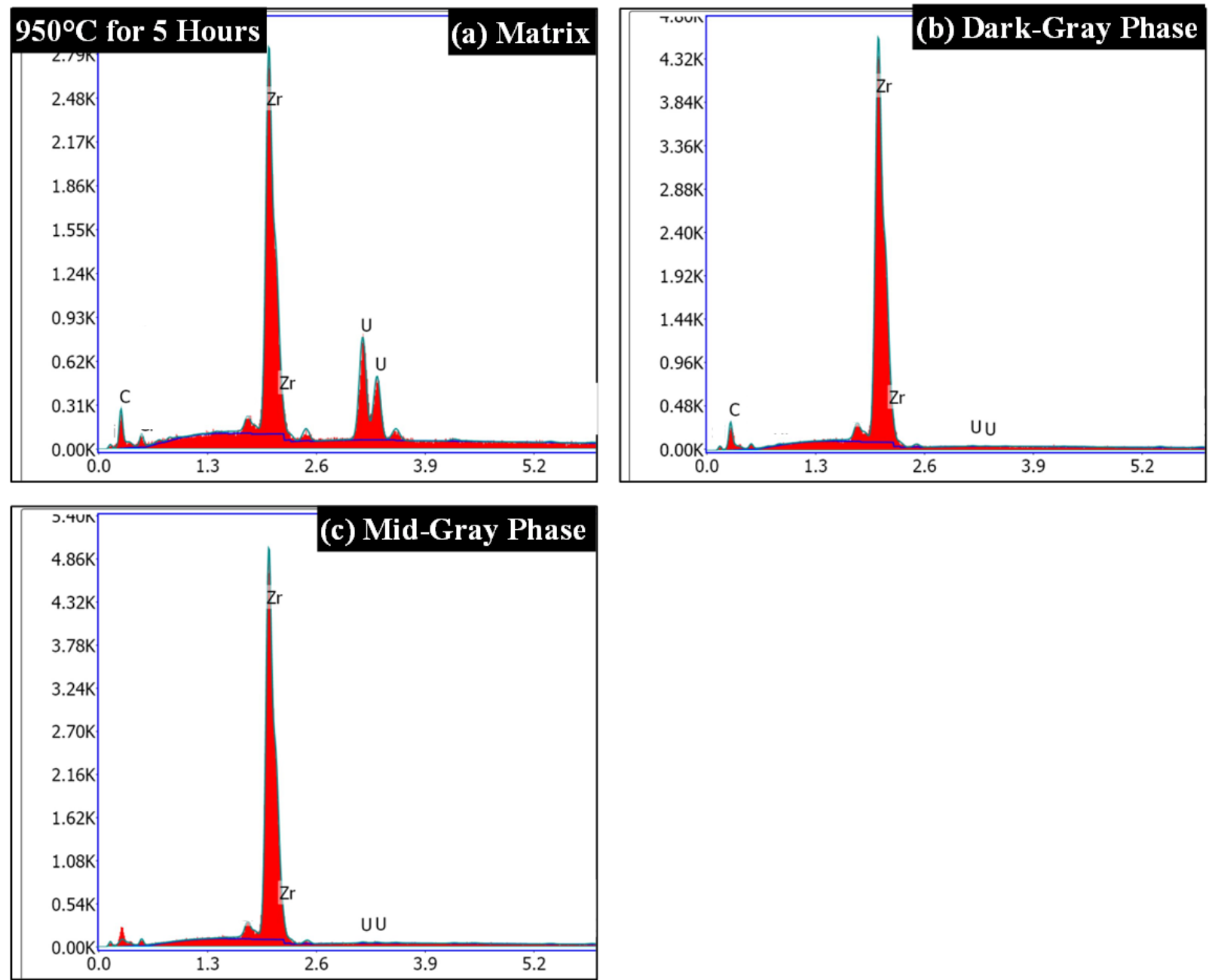

Figure 38. Typical EDS patterns for (a) matrix, (b) dark-gray phase, and (c) mid-gray phase regions in fuel sample annealed at $950^{\circ} \mathrm{C}$ for 5 hours.

Table 1. Measured concentrations of different observed features in fuel microstructure of sample annealed at $950^{\circ} \mathrm{C}$ for 5 hours.

\begin{tabular}{|c|c|c|c|}
\hline \multirow{2}{*}{ Element } & Matrix & Mid-Gray Phase & Dark-Gray Phase \\
\cline { 2 - 4 } & \multicolumn{3}{|c|}{ Concentration (at.\%) } \\
\hline $\mathrm{Er}$ & $1.2 \pm 0.4$ & $0.6 \pm 0.2$ & $0.6 \pm 0.1$ \\
\hline $\mathrm{U}$ & $20 \pm 1.3$ & $0.4 \pm 0.2$ & $0.2 \pm 0.1$ \\
\hline $\mathrm{Zr}$ & $78.8 \pm 1.3$ & $99.0 \pm 0.2$ & $99.2 \pm 0.1$ \\
\hline
\end{tabular}



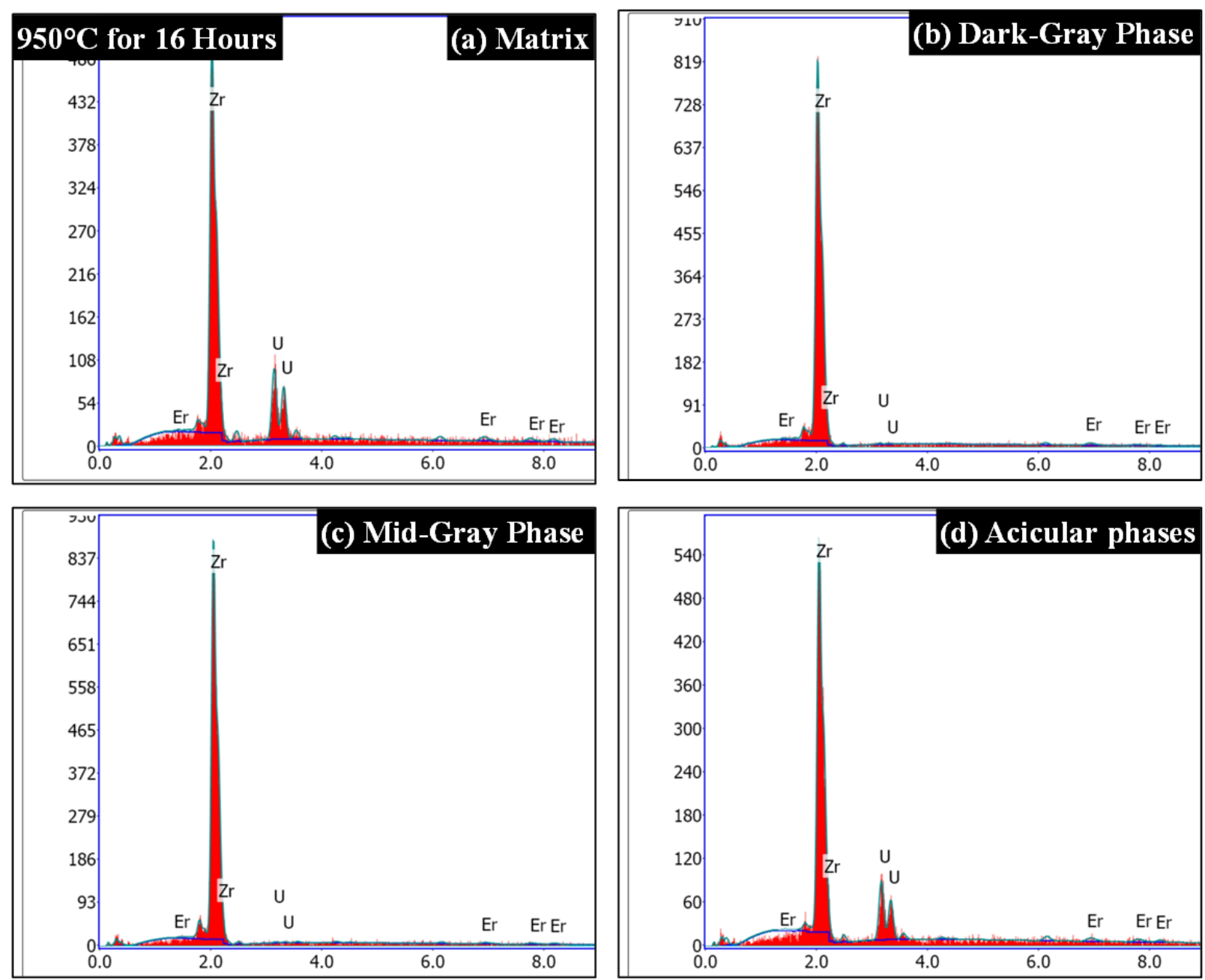

Figure 39. Typical EDS patterns for (a) matrix, (b) dark-gray phase, (c) mid-gray phase, and (d) acicular regions in fuel sample annealed at $950^{\circ} \mathrm{C}$ for 16 hours.

Table 2. Measured concentrations of different observed features in fuel microstructure of sample annealed at $950^{\circ} \mathrm{C}$ for 16 hours.

\begin{tabular}{|c|c|c|c|c|}
\hline \multirow{2}{*}{ Element } & Matrix & Mid-Gray Phase & Dark-Gray Phase & Grain Boundaries \\
\cline { 2 - 5 } & \multicolumn{4}{|c|}{ Concentration (at.\%) } \\
\hline $\mathrm{Er}$ & $1.5 \pm 0.7$ & $0.8 \pm 0.3$ & $1.0 \pm 0.1$ & $1.4 \pm 0.6$ \\
\hline $\mathrm{U}$ & $16.0 \pm 2.5$ & $4.8 \pm 0.6$ & $4.0 \pm 0.5$ & $13.3 \pm 2.6$ \\
\hline $\mathrm{Zr}$ & $82.5 \pm 3.1$ & $94.3 \pm 0.7$ & $95.5 \pm 0.5$ & $85.3 \pm 2.5$ \\
\hline
\end{tabular}



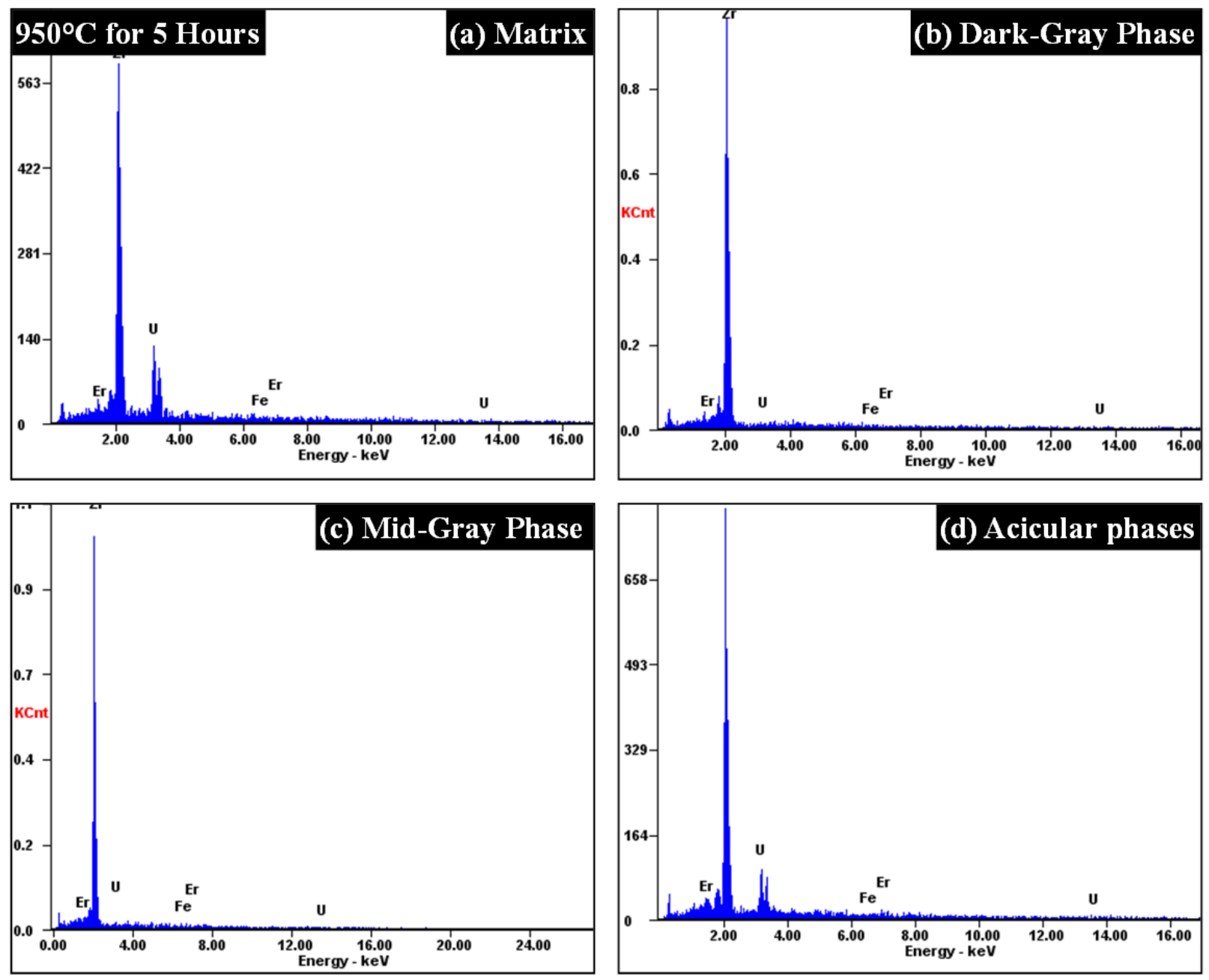

Figure 40. Typical EDS patterns for (a) matrix, (b) dark-gray phase, (c) mid-gray phase, and (d) acicular regions in fuel sample annealed at $950^{\circ} \mathrm{C}$ for 24 hours.

Table 3. Measured concentrations of different observed features in fuel microstructure of sample annealed at $950^{\circ} \mathrm{C}$ for 24 hours.

\begin{tabular}{|c|c|c|c|c|}
\hline \multirow{2}{*}{ Element } & Matrix & Mid-Gray Phase & Dark-Gray Phase & Grain Boundaries \\
\cline { 2 - 5 } & \multicolumn{4}{|c|}{ Concentration (at.\%) } \\
\hline $\mathrm{Er}$ & $2.5 \pm 0.3$ & $2.5 \pm 0.9$ & $1.5 \pm 0.3$ & $3.0 \pm 0.8$ \\
\hline $\mathrm{U}$ & $14.0 \pm 0.1$ & $0.7 \pm 0.3$ & $0.6 \pm 0.3$ & $10.8 \pm 1.1$ \\
\hline $\mathrm{Zr}$ & $81.4 \pm 0.2$ & $94.9 \pm 1.0$ & $95.9 \pm 0.4$ & $84.1 \pm 0.4$ \\
\hline
\end{tabular}




\subsubsection{Characterization by Transmission Electron Microscopy}

To develop a better understanding of the phase evolution in the fuel as a result of annealing at $950^{\circ} \mathrm{C}$, a sample was prepared for characterization by TEM from the sample annealed for 24 hours. A region intersecting an acicular feature and the matrix was selected for analysis, as shown in Figure 41(a). The prepared TEM sample is shown in the HAADF micrograph in Figure 41(b). Due to time constraints, analysis of the other features observed in the samples annealed at $950^{\circ} \mathrm{C}$ was not conducted by TEM. Characterization of these additional features in the microstructure (the dark- and mid-gray features observed in the SEM BE micrographs) remains a source for future studies.

The mid-gray acicular phase in Figure 41(a) appears as a light-contrast secondary phase in the HAADF micrograph in Figure 41(b). The fuel matrix appears as the darker-contrast phase. EDS patterns from the matrix and secondary phase are shown in Figure 42(a) and Figure 42(b), respectively. The patterns agree with those shown in Figure 40, collected in the SEM.
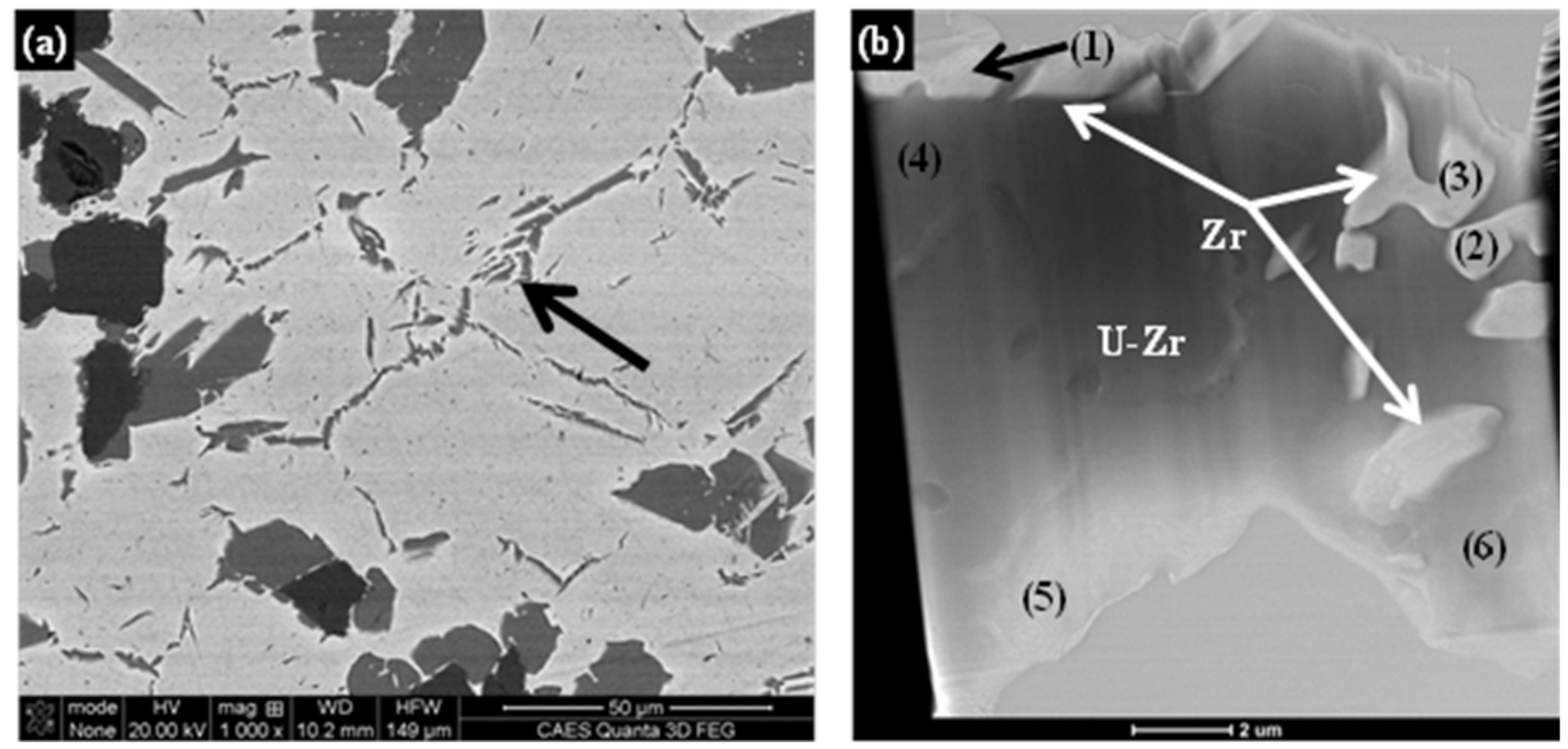

Figure 41. (a) BE micrograph illustrating the region selected (marked by an arrow) for characterization by TEM. (b) HAADF micrograph of the prepared TEM sample.
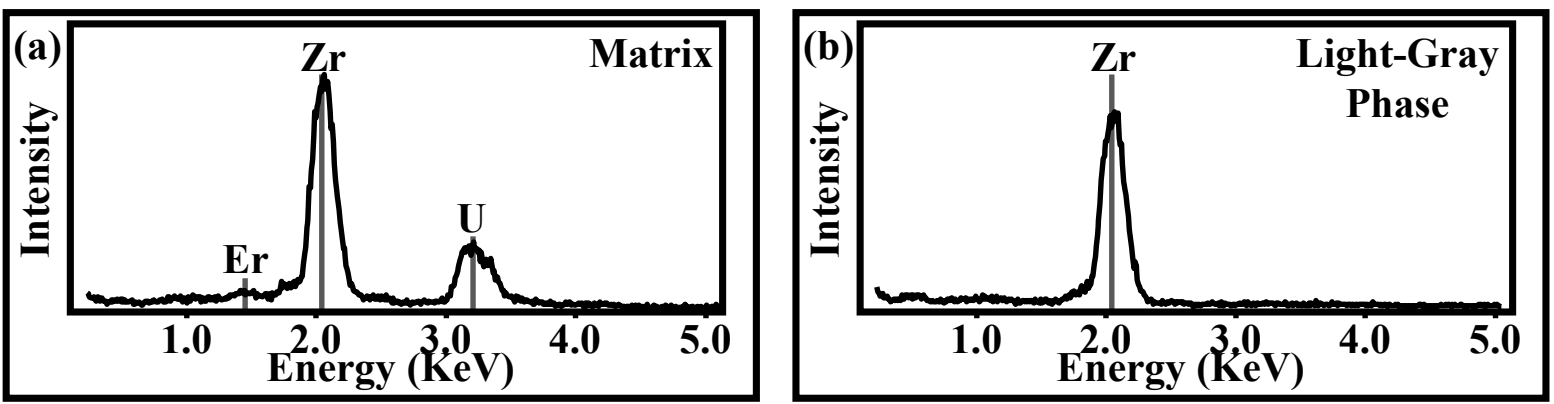

Figure 42. EDS patterns of the (a) matrix and the (b) secondary phase captured in the TEM sample in Figure 41(b).

Diffraction analysis was carried out on the different phase regions in the TEM sample. The same method employed to identify hydride phases in the as-received samples was used in this characterization. Because hydrogen cannot be detected by EDS, likely hydride and non-hydride phases were considered as 
candidate phases during the analysis. The diffraction spot patterns calculated from the crystallographic data were compared to the experimental patterns obtained from the different phase regions in the TEM sample. For this sample, the tetragonal- $\mathrm{H}_{2} \mathrm{Zr}$ phase (tI6, I4/mmm), cubic- $\mathrm{H}_{1.6} \mathrm{Zr}(\mathrm{cF} 12, \mathrm{Fm}-3 \mathrm{~m})$, hexagonal- $\alpha-\mathrm{Zr}$ (hP2, $\left.\mathrm{P}_{3} / \mathrm{mmc}\right)$, cubic- $\mathrm{U}_{0.3} \mathrm{Zr}_{0.7}(\mathrm{cI} 2, \mathrm{Im}-3 \mathrm{~m})$, and hexagonal- $\mathrm{U}_{0.87} \mathrm{Zr}_{2.13}\left(\mathrm{UZr}_{2}, \mathrm{hP} 3\right.$, $\mathrm{P} 6 / \mathrm{mmm}$ ) phases were considered in the analysis. The analysis was complicated by significant similarities exhibited in the diffraction patterns generated by these phases.

In most cases, the diffraction patterns collected from the sample closely matched those generated by more than one of the phases considered. The closest match was identified as the phase present in the sample. An example of the pattern similarities is shown in Figure 43, which depicts the diffraction patterns generated from Grain 1, as marked in Figure 41 and shown in Figure 44. Three zone axes are shown. The collected patterns without indexing data are shown on the left of the figure and are labeled Zones 1, 2, and 3. The two patterns next to each zone axes represent the indexing results from the JEMS ${ }^{\mathrm{TM}}$ software. Note that each zone axes closely matches the $\mathrm{H}_{1.6} \mathrm{Zr}$ and $\mathrm{H}_{2} \mathrm{Zr}$ phases. The calculated patterns exhibit deviation from the experimental pattern. Ideal pattern matches were not achieved. The deviations may be the result of variations in hydrogen concentrations in the two phases considered due to solid solutioning. Solid solutioning results in variations of the unit cell lattice parameters, which may be expressed in the diffraction patterns as changes in the d-spacing.

Close examination of each of the fitted patterns showed that the $\mathrm{H}_{2} \mathrm{Zr}$ phase presented a closer pattern match. As a result, the $\mathrm{H}_{2} \mathrm{Zr}$ phase was selected as the phase present in the fuel sample. Note the actual hydrogen content in the grain was not known, and that solid solution of hydrogen in the phases considered in this example, and others considered below for other grains, can significantly affect the lattice parameters. For the case in Figure 43, the experimental pattern can be matched to both phases as long as the hydrogen content of the actual grain analyzed is not known. Thus, some error may be present in the conclusions in this study. However, significant certainty remains that hydrogen is present in the grains considered: the experimental diffraction patterns did not match the $\alpha$-Zr phase, which would be the stable $\mathrm{Zr}$ phase from the annealing temperature down to room temperature. The $\beta-\mathrm{Zr}$ phase presented a good match. The $\mathrm{H}-\mathrm{Zr}$ phase diagram indicates that the $\beta-\mathrm{Zr} \rightarrow \alpha-\mathrm{Zr}$ phase transformation starts, at least, from 863 to $550^{\circ} \mathrm{C}$ (the phase diagram is incomplete), depending on hydrogen concentration. Because the furnace temperature decreased at a relatively slow rate, as shown in Figure 31(a), (b) and (c), the $\beta-\mathrm{Zr}$ phase was not expected to be retained upon cooling. Thus, this phase was not considered for further analysis. 

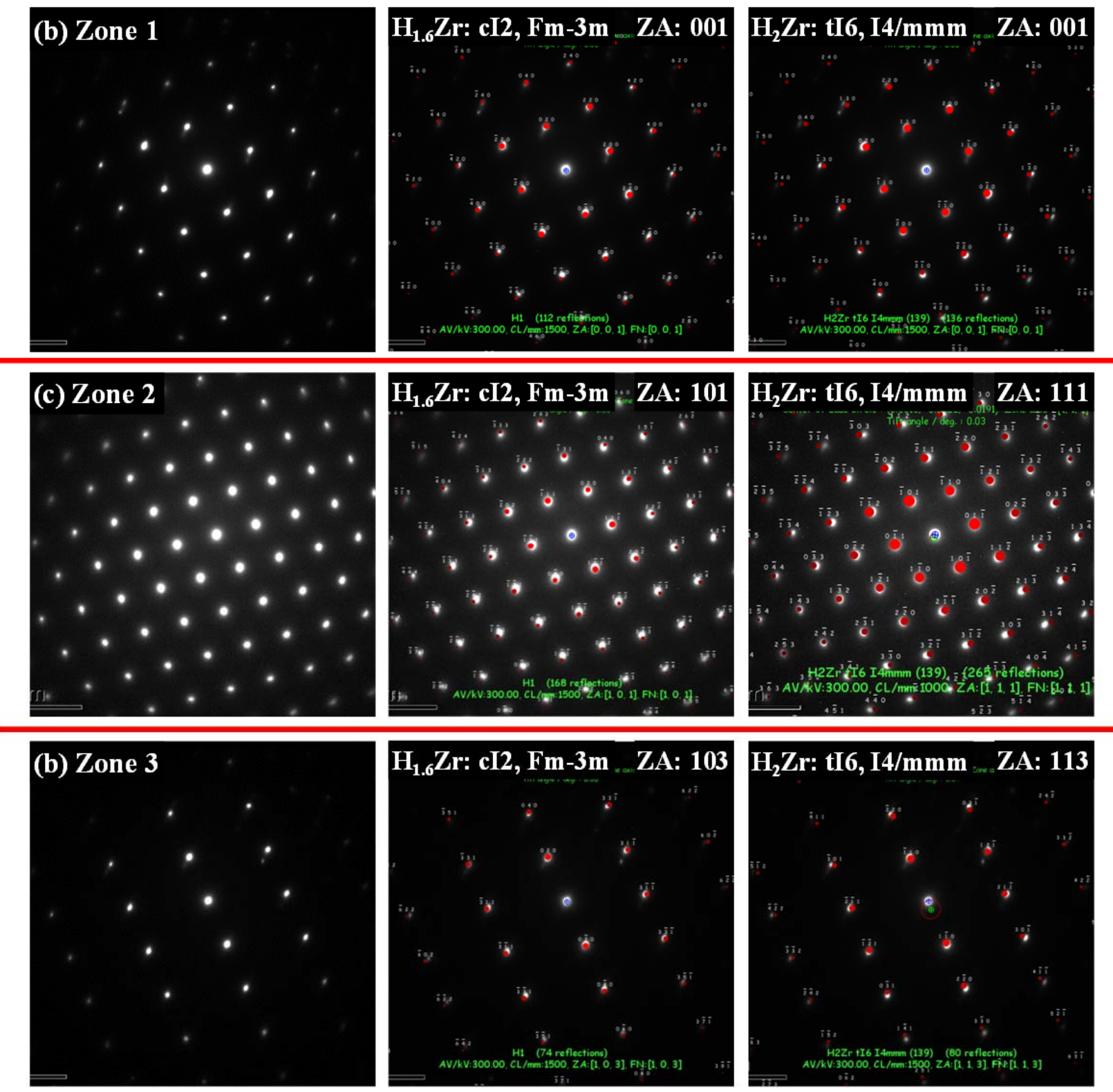

Figure 43. Example of diffraction patterns from Grain 1 depicting three distinct zone axes. Each zone axis closely matches the $\mathrm{H}_{1.6} \mathrm{Zr}$ and $\mathrm{H}_{2} \mathrm{Zr}$ phases. The $\mathrm{H}_{2} \mathrm{Zr}$ phase presents a closer pattern match.

Characterization of the TEM sample annealed at $950^{\circ} \mathrm{C}$ for 24 hours was carried out on the matrix and secondary phases captured in the sample. Diffraction analysis on Grain 1, as labeled in Figure 40(b), revealed that the acicular phase shown in Figure 34 and Figure 41, that was identified to contain only $\mathrm{Zr}$ in Figure 42, is composed of the tetragonal- $\mathrm{H}_{2} \mathrm{Zr}$ phase, as summarized in Figure 44. Similarly, diffraction analysis on Grains 2 and 3, in the sample determined that they contained the same phase.

Characterization was carried out on the matrix at a few locations, labeled Grains 4, 5, and 6 in Figure 41. Grain 4 closely matched the hexagonal- $\mathrm{UZr}_{2}$ phase (indexed as $\mathrm{U}_{0.87} \mathrm{Zr}_{2.13}$ ), as indexed in Figure 45. However, Grains 5 and 6 matched both the hexagonal- $\mathrm{UZr}_{2}$ and cubic- $\mathrm{U}_{0.3} \mathrm{Zr}_{0.7}$ phases with equal certainty. The EDS patterns collected from different locations of the matrix within the sample showed nearly identical $U$ concentrations. Thus, this study tentatively assumes that Grains 5 and 6 also contain the $\mathrm{UZr}_{2}$ phase. Further analysis is necessary to distinguish more clearly between the phases. 
Note that the diffraction patterns for these grains also showed close relationships to the tetragonal- $\mathrm{H}_{2} \mathrm{Zr}$ and cubic- $\mathrm{H}_{1.6} \mathrm{Zr}$ phases. These were excluded because these phases lack $\mathrm{U}$, and the EDS patterns, as shown in Figure 42(a), show that the grains under consideration contained significant U concentrations.
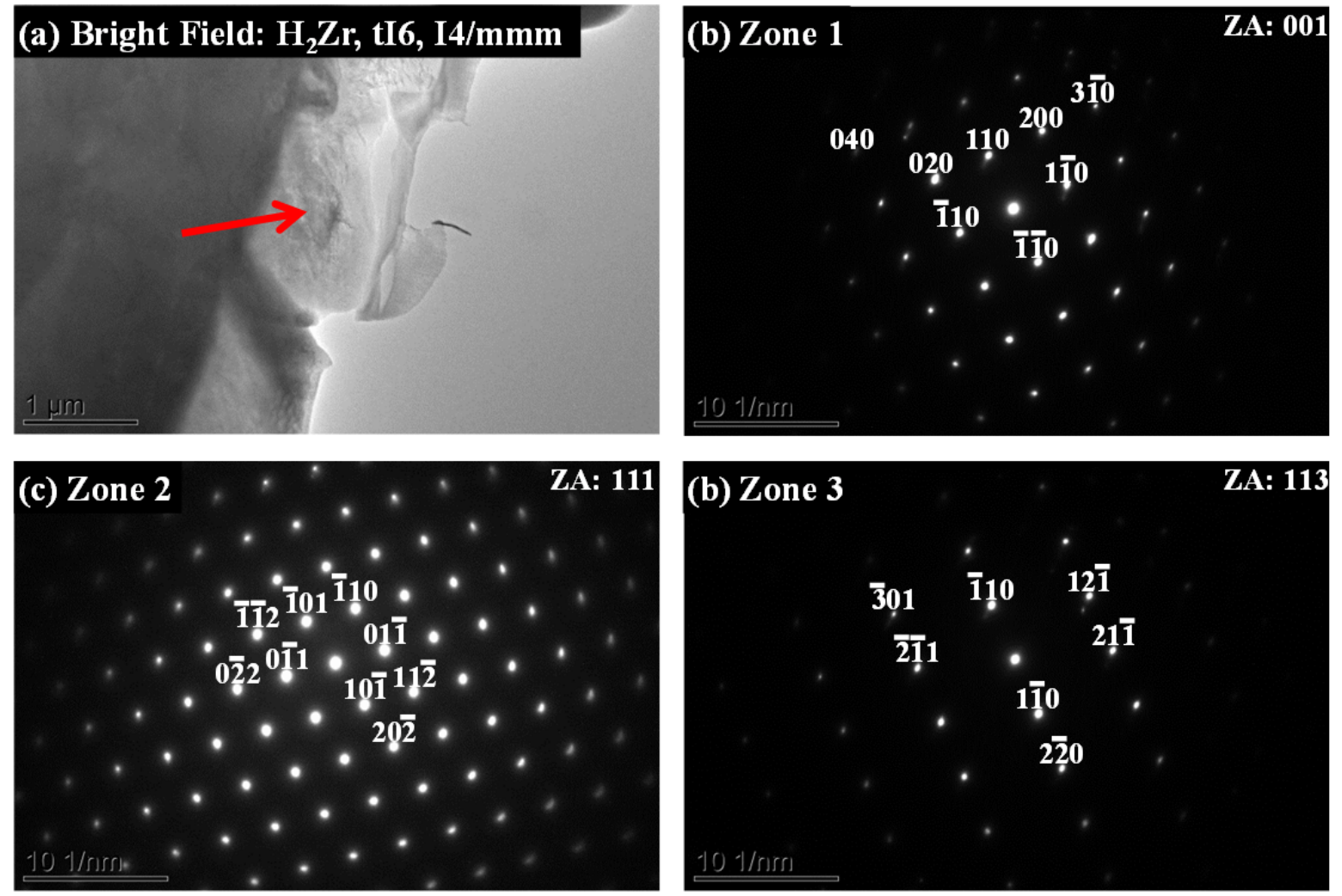

Figure 44. (a) Bright field micrograph showing a grain, marked by a red arrow, selected for analysis (Grain 1). (b), (c), and (d) Electron diffraction analysis showing three selected zone axes collected from the grain, identifying the tetragonal- $\mathrm{H}_{2} \mathrm{Zr}$ phase (tI6, I4/mmm). 

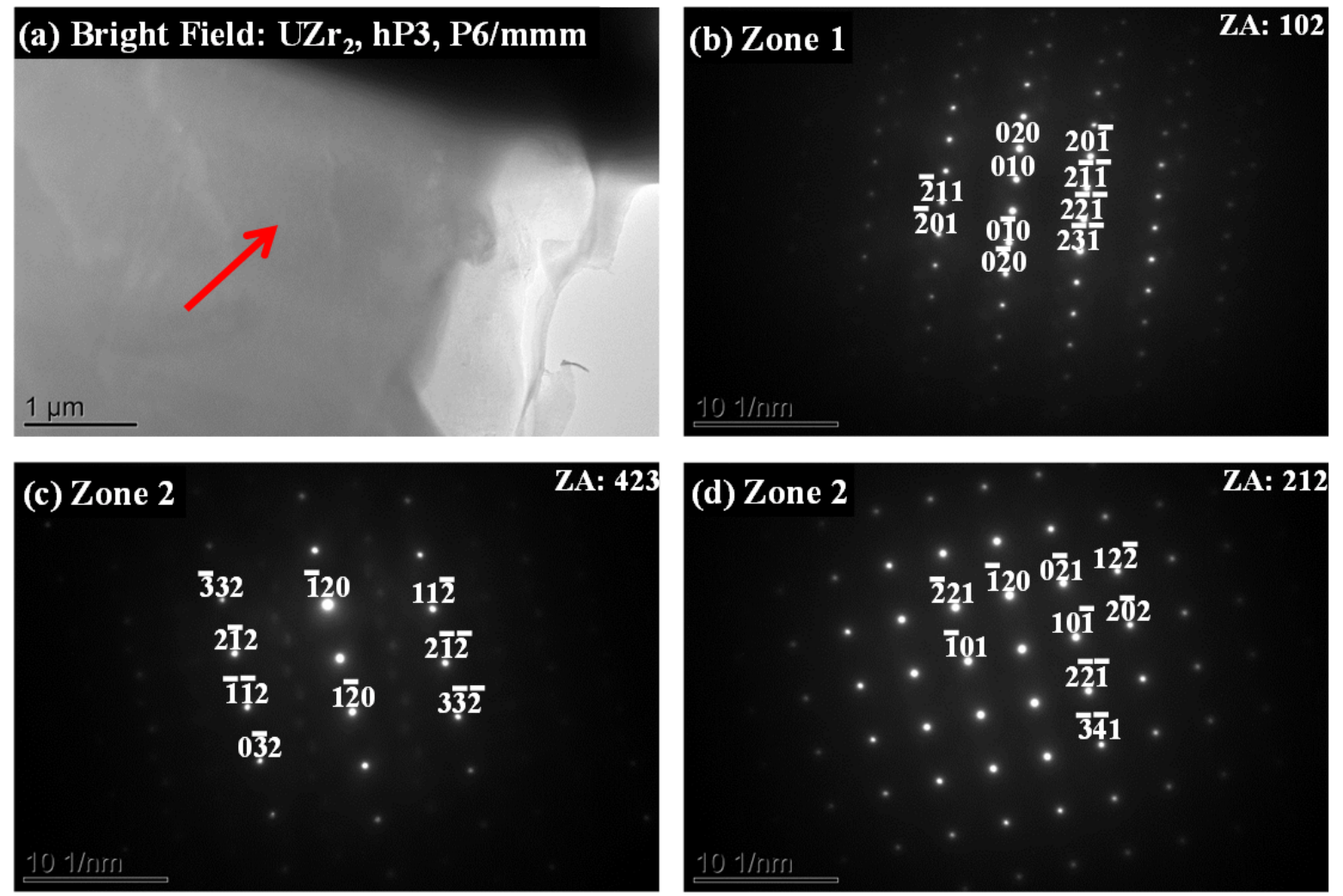

Figure 45. (a) Bright field micrograph showing a grain, marked by a red arrow, selected for analysis; (b), (c), and (d) Electron diffraction analysis showing three selected zone axes collected from the grain, identifying the hexagonal- $\mathrm{UZr}_{2}$ phase $(\mathrm{hP} 3, \mathrm{P} 6 / \mathrm{mmm})$.

\subsubsection{Diffusion Couple Experiments}

Diffusion studies were carried out to develop an understanding of the interactions that can take place at high temperature between TRIGA fuels and the 304SS cladding used to shield the fuel. To accomplish this goal, diffusion couples of TRIGA fuel versus $304 \mathrm{SS}$ were assembled and were annealed at $1000^{\circ} \mathrm{C}$ for 5 and 24 hours.

The diffusion couples typically suffered from lack of bonding or early failure of the bonding interface. The likely cause of this failure is discussed below. Where bonding was achieved, interaction regions developed within the 304SS. The interaction regions underwent characterization by SEM. The interaction region in the diffusion couple annealed for 5 hours was characterized in more detail by TEM to obtain crystallographic information so that the phases present in the interaction regions could be identified.

During annealing of the diffusion couples, two thermocouples were inserted into the furnace to monitor the furnace temperature as a function of time. Figure 46 shows the furnace temperature conditions as a function of time for the fuel samples annealed at $1000^{\circ} \mathrm{C}$ for (a) 5 hours and (b) 24 hours. The initial 9600 seconds (2:40 hours) of data in the graph for the diffusion couple annealed for 5 hours was lost due to a computer glitch. The dashed yellow line estimates the lost data by extrapolation from the furnace controller programing. 
The profiles from each thermocouple in both graphs are nearly identical. The temperature profiles were plotted in magenta and blue color. The blue colored profiles lay below the magenta colored ones. The line thickness for the profile displayed in blue was thickened so that it is visible in the graph. The thermocouple data show that the furnace experienced negligible temperature variations during annealing at the two specified annealing times.
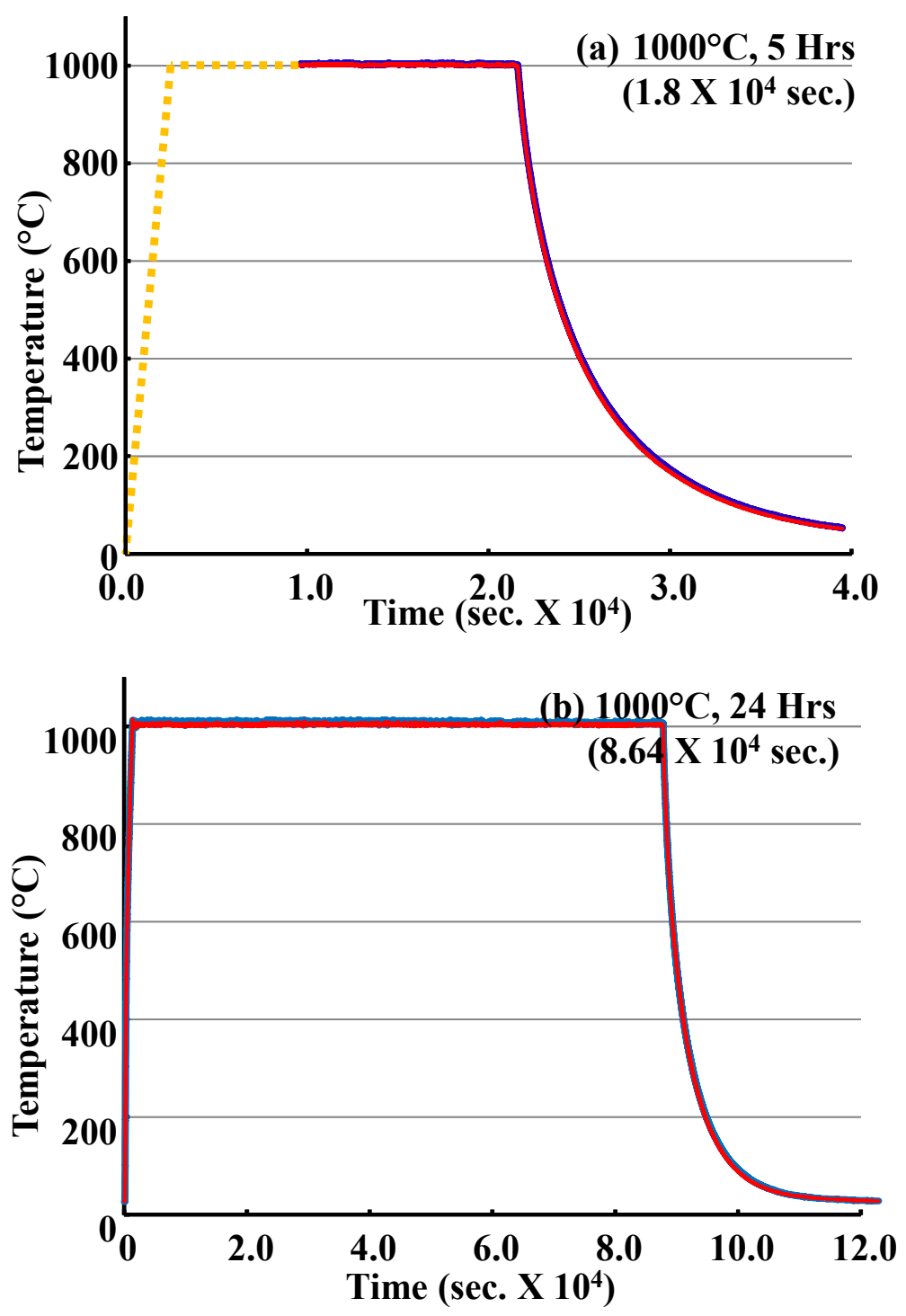

Figure 46. Temperature profiles generated from TRIGA fuel annealing process at $1000^{\circ} \mathrm{C}$ for (a) 5 hours and (b) 24 hours.

\subsubsection{Characterization by Scanning Electron Microscopy}

The diffusion couple annealed at $1000^{\circ} \mathrm{C}$ for 5 hours developed a small discontinuous interaction region as shown on the BE micrograph in Figure 47(a). Figure 47(b) depicts the interaction region in more detail. Based on phase contrast several phases developed within the interaction region. Localized regions with similar compositions, microstructures and/or number of phases are also apparent within the interaction region. These regions reoccurred throughout the width of the interaction region and at similar distributions through its thickness. From here on, these localized features in the microstructure of the interaction region will be referred to as phase regions. EDS analysis was performed on the 304SS near the 
interaction region, within the interaction region, and on the TRIGA fuel near the interaction region. Figure 48(a) shows a BE micrograph of the interaction region identifying, based on backscatter phase contrast, the differing phase observed regions. The EDS patterns in Figure 48(b) through (f) qualitatively show their average compositions, and Table 4 shows the quantified data. The nominal composition of $304 \mathrm{SS}$ in weight percent is $\mathrm{Fe}-18.3 \mathrm{Cr}-8.2 \mathrm{Ni}-1.8 \mathrm{Mn}-0.7 \mathrm{Cu}-0.5 \mathrm{Mo}-0.3 \mathrm{Si}-0.2 \mathrm{Co}$. Elements from the TRIGA fuel penetrated into the 304SS to a depth of approximately $15 \mu \mathrm{m}$, as shown in Figure 47(b) and Figure 48(a), resulting in the development of the interaction region.
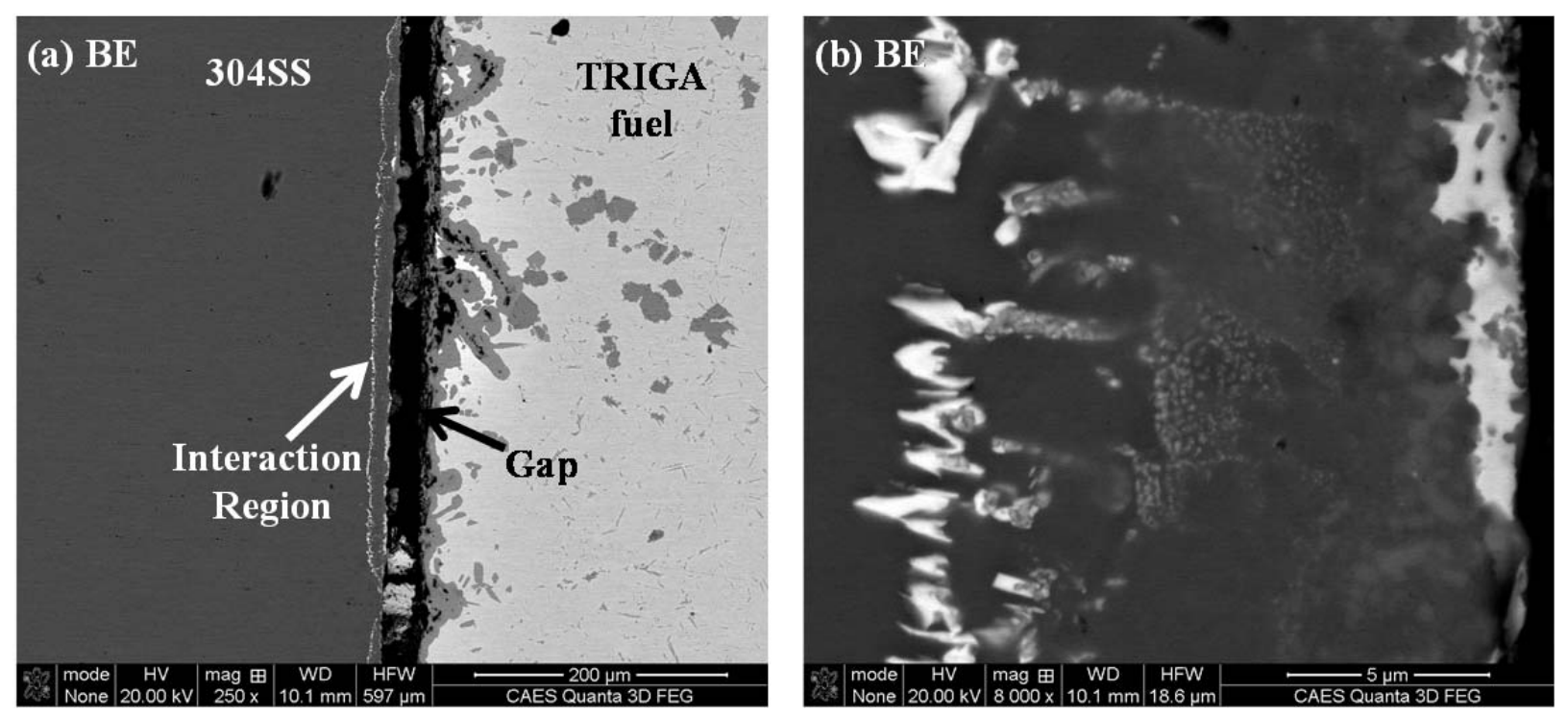

Figure 47. Backscatter electron micrographs of (a) a selected region from the TRIGA fuel versus 304SS diffusion couple annealed at $1000^{\circ} \mathrm{C}$ for 5 hours, and (b) a detailed micrograph of the interaction region. 

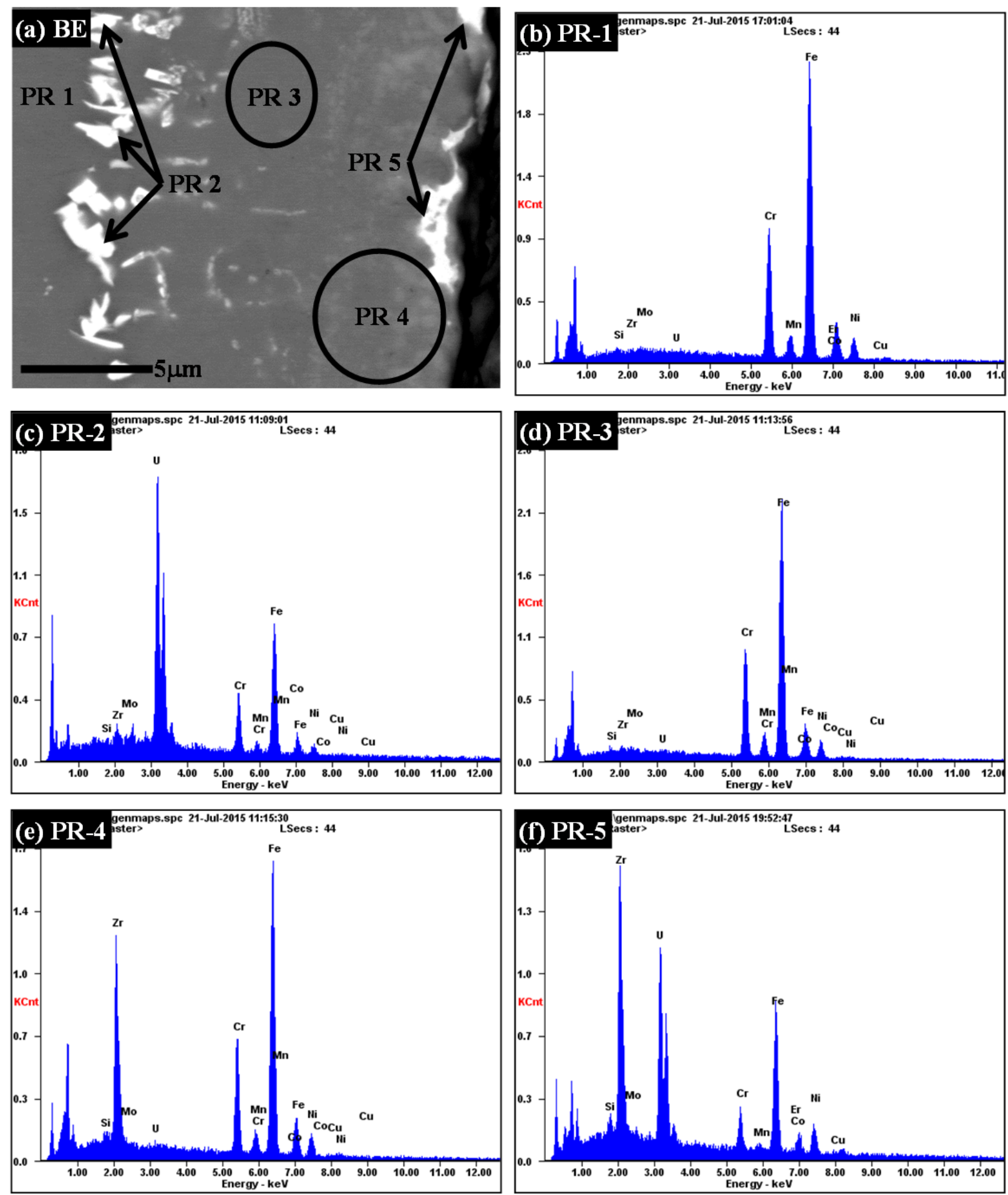

Figure 48. (a) Backscatter electron micrograph of interaction region in diffusion couple annealed for 5 hours, tentatively showing several phases and phase regions (PR). (b) through (e) Typical EDS patterns for the differing phase regions in the interaction region where (b) PR-1 is the 304SS away from the interaction region, (c) PR-2 is a U-Fe-Cr-Ni-rich phase in the interaction region, (d) PR-3 is a Fe-Cr-Ni-rich phase region, (e) PR-4 is a Zr-Fe-Cr-Ni-rich phase region, and (f) PR-5 is a $\mathrm{U}-\mathrm{Zr}-\mathrm{Fe}-\mathrm{Cr}-\mathrm{Ni}$-rich phase region. 
Table 4. Measured concentrations of the different phase regions observed in the interaction region of the diffusion couple annealed at $1000^{\circ} \mathrm{C}$ for 5 hours, as labeled in Figure 48.

\begin{tabular}{|c|c|c|c|c|c|}
\hline \multirow{2}{*}{ Element } & PR-1 & PR-2 & PR-3 & PR-4 & PR-5 \\
\cline { 2 - 6 } & \multicolumn{5}{|c|}{ Concentration (at.\%) } \\
\hline $\mathrm{Er}$ & $0.2 \pm 0.2$ & 0 & 0 & 0 & $0.2 \pm 0.3$ \\
\hline $\mathrm{U}$ & $0.1 \pm 0.0$ & $30.5 \pm 16.1$ & $0.2 \pm 0.0$ & $1.3 \pm 2.6$ & $17.5 \pm 4.5$ \\
\hline $\mathrm{Zr}$ & $0.4 \pm 0.0$ & $5.8 \pm 5.7$ & $1.0 \pm 0.5$ & $13.8 \pm 7.2$ & $21.4 \pm 3.1$ \\
\hline $\mathrm{Cr}$ & $19.3 \pm 0.3$ & $12.4 \pm 4.9$ & $21.3 \pm 3.8$ & $16.3 \pm 1.7$ & $4.8 \pm 3.1$ \\
\hline $\mathrm{Fe}$ & $68.4 \pm 0.0$ & $39.2 \pm 13.8$ & $66.4 \pm 2.4$ & $57.1 \pm 6.8$ & $37.4 \pm 0.8$ \\
\hline $\mathrm{Ni}$ & $7.2 \pm 0.1$ & $6.0 \pm 3.9$ & $5.9 \pm 1.8$ & $6.7 \pm 0.8$ & $14.2 \pm 3.3$ \\
\hline $\mathrm{Co}$ & $0.4 \pm 0.3$ & $1.3 \pm 0.7$ & $1.0 \pm 0.3$ & $0.7 \pm 0.1$ & $0.9 \pm 0.2$ \\
\hline $\mathrm{Cu}$ & $0.7 \pm 0.0$ & $1.3 \pm 0.5$ & $0.8 \pm 0.3$ & $0.8 \pm 0.2$ & $1.3 \pm 0.3$ \\
\hline $\mathrm{Mn}$ & $1.7 \pm 0.2$ & $1.6 \pm 0.3$ & $1.8 \pm 0.4$ & $1.7 \pm 0.2$ & $1.5 \pm 0.2$ \\
\hline $\mathrm{Mo}$ & $0.5 \pm 0.1$ & $0.8 \pm 0.5$ & $0.6 \pm 0.2$ & $0.5 \pm 0.1$ & $0.3 \pm 0.4$ \\
\hline $\mathrm{Si}$ & $1.1 \pm 0.1$ & $1.2 \pm 0.6$ & $1.1 \pm 0.3$ & $1.0 \pm 0.7$ & $0.7 \pm 1.0$ \\
\hline
\end{tabular}

EDS confirmed that interaction products were found only within the 304SS alloy. The BE micrograph in Figure 49(a) shows the TRIGA fuel in the diffusion couple at a section adjacent to the interaction region. The EDS patterns in Figure 49(b) though (d) show three distinct compositions. Qualitative analysis of the figures and quantified data in Table 5 confirm that elements from the 304SS do not appear to have diffused into the TRIGA fuel side of the diffusion couple in measurable concentrations. A notable feature, as can be observed in the SE micrograph in Figure 50, is that the TRIGA fuel typically developed voids in the Zr-rich regions wherever an interaction region developed in the adjacent 304SS. The cause of these voids was not considered in this study and requires further examination.

Note that the EDS data in this report was quantified through standardless analysis. Elements in low concentrations (approximately $<1$ at.\%) carry a significant error, and their concentrations may be assumed to be zero. Concentrations that were measured approximately 1 at.\% or less were included in Table 4 and Table 5 for completion. 

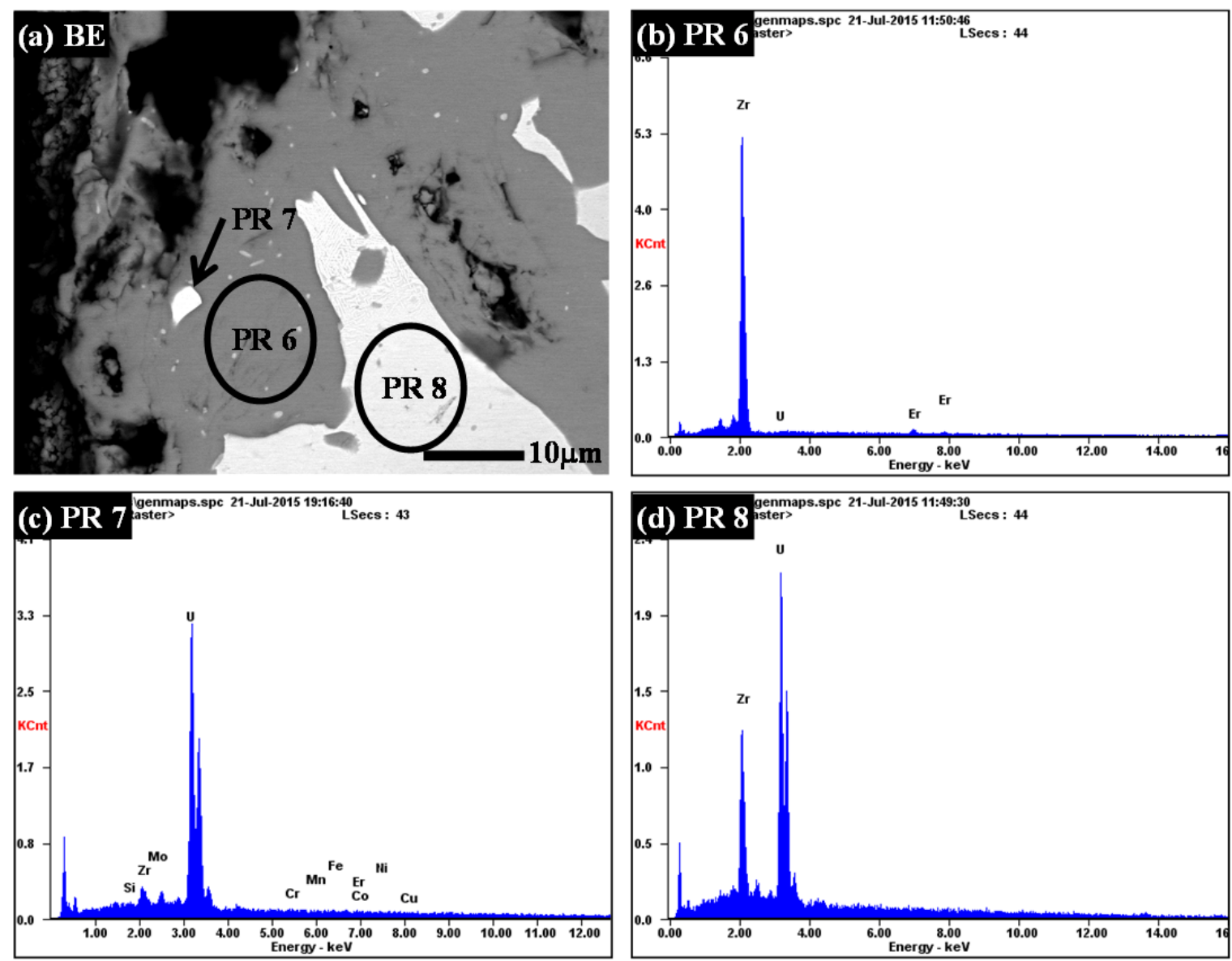

Figure 49. (a) Backscatter electron micrograph of the TRIGA fuel near the interaction region, in the diffusion couple annealed at $1000^{\circ} \mathrm{C}$ for 5 hours, tentatively showing three phases. (b) through (d) show typical qualitative EDS patterns for the differing phase regions (PR) in the interaction region where (b) PR-6 contains only Zr, (c) PR-7 is nearly pure U-phase, and (d) PR-8 is the fuel matrix.

Table 5. Measured concentrations of the different phase regions observed in the TRIGA fuel near the interaction region of the diffusion couple annealed at $1000^{\circ} \mathrm{C}$ for 5 hours, as labeled in Figure 49.

\begin{tabular}{|c|c|c|c|}
\hline \multirow{2}{*}{ Element } & PR-6 & PR-7 & PR-8 \\
\cline { 2 - 4 } & \multicolumn{3}{|c|}{ Concentration (at.\%) } \\
\hline $\mathrm{Er}$ & $1.4 \pm 1.7$ & $1.2 \pm 0.3$ & $0.8 \pm 0.5$ \\
\hline $\mathrm{U}$ & $0.6 \pm 0.4$ & $58.9 \pm 13.7$ & $43.9 \pm 10.5$ \\
\hline $\mathrm{Zr}$ & $92.9 \pm 4.3$ & $29.3 \pm 18.1$ & $52.4 \pm 9.6$ \\
\hline $\mathrm{Cr}$ & $1.0 \pm 0.7$ & $1.5 \pm 0.3$ & $1.2 \pm 0.0$ \\
\hline $\mathrm{Fe}$ & $2.0 \pm 1.8$ & $2.0 \pm 0.7$ & $1.6 \pm 0.2$ \\
\hline $\mathrm{Ni}$ & $0.9 \pm 0.4$ & $1.4 \pm 1.2$ & $1.7 \pm 0.3$ \\
\hline $\mathrm{Co}$ & $0.3 \pm 0.4$ & $0.9 \pm 0.3$ & $1.1 \pm 0.9$ \\
\hline $\mathrm{Cu}$ & $0.7 \pm 0.3$ & $1.7 \pm 0.9$ & $1.8 \pm 0.1$ \\
\hline $\mathrm{Mn}$ & $0.5 \pm 0.1$ & $1.2 \pm 0.6$ & $0.7 \pm 0.6$ \\
\hline $\mathrm{Mo}$ & $0.6 \pm 0.4$ & $1.0 \pm 0.9$ & $0.3 \pm 0.4$ \\
\hline $\mathrm{Si}$ & $0.6 \pm 0.1$ & $0.8 \pm 1.1$ & 0 \\
\hline
\end{tabular}




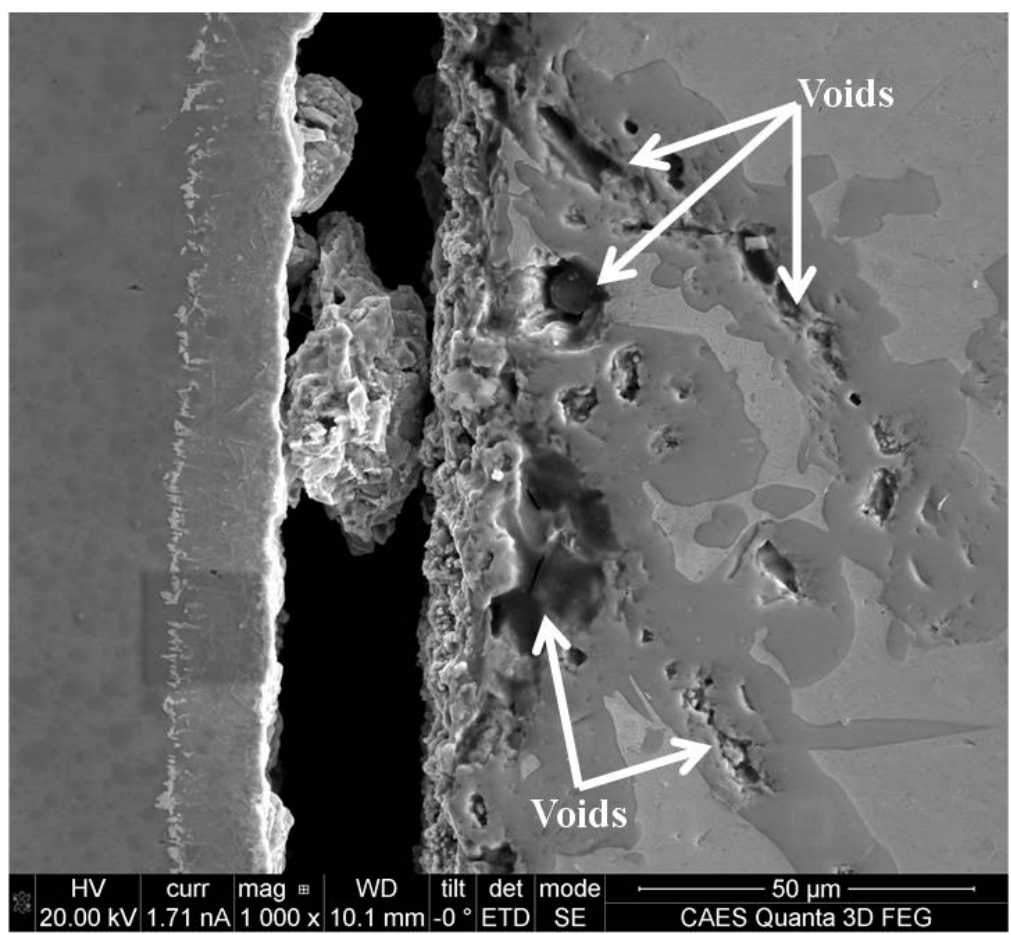

Figure 50. Secondary electron micrograph displaying voids within TRIGA fuel near interaction region of diffusion couple.

X-ray maps were generated from a selected section of the interaction region to qualitatively identify the presence and location of the elements from the fuel and 304SS, and to obtain a more generalized element distribution than that provided by the EDS analysis. The x-ray maps are in good agreement with the EDS analysis. Nonetheless, the maps show a composition, not identified by EDS, near the center of the interaction region and within PR-3 that contains higher concentrations of $\mathrm{Cr}, \mathrm{Fe}$, and $\mathrm{Mn}$ with lower concentrations of $\mathrm{Ni}$ than the 304SS, and likely differs from the Fe-Cr-Ni phase composition identified as PR-3 in the EDS analysis. The maps also suggest that the U-Fe-Cr-Ni-rich phase identified as PR-2 in the EDS analysis is also somewhat enriched with copper. 

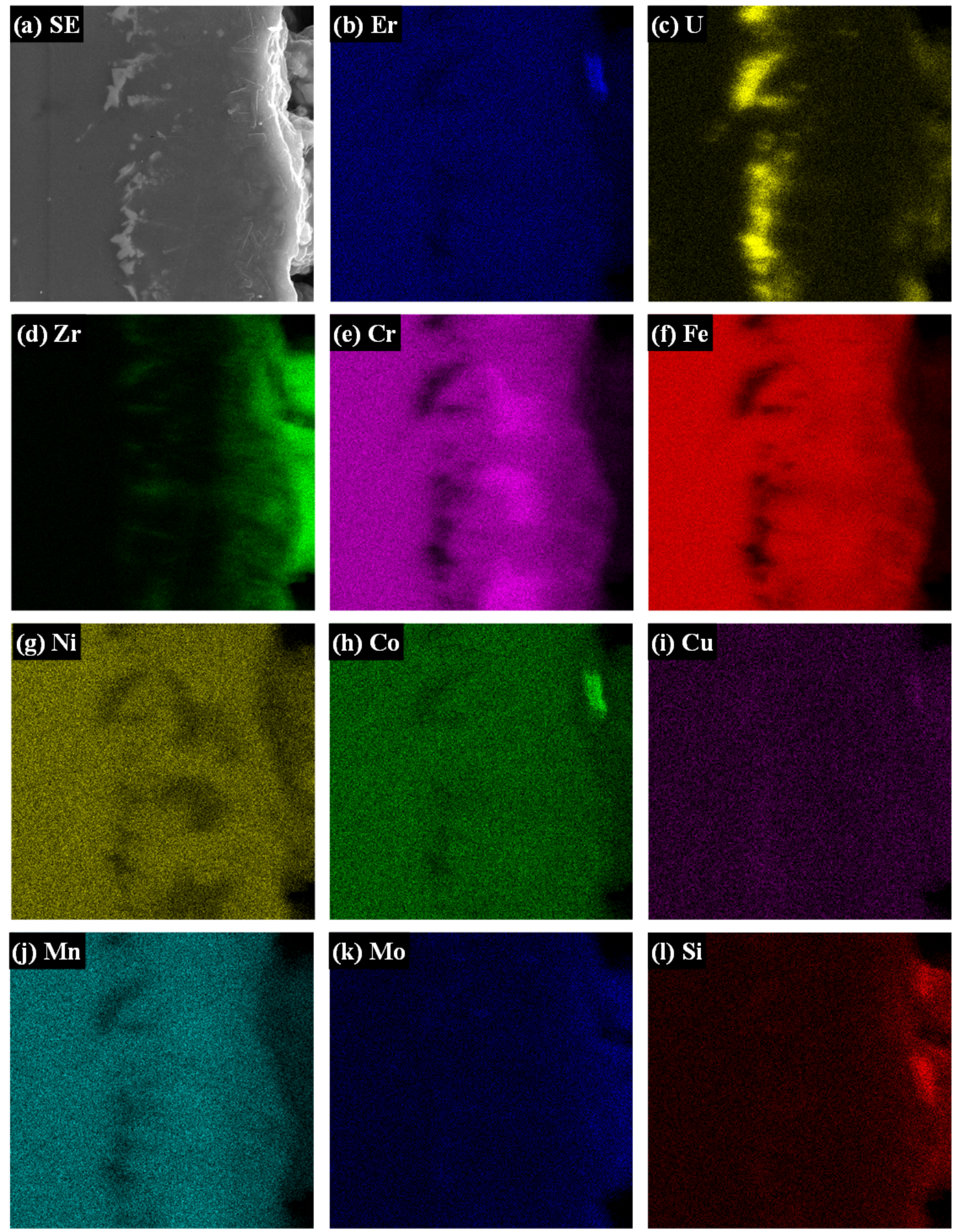

Figure 51. (a) SE micrograph of the interaction region in the diffusion couple annealed at the $1000^{\circ} \mathrm{C}$ for 5 hours, and x-ray maps of the region qualitatively showing the presence and location of (b) Er, (c) U, (d) $\mathrm{Zr}$, (e) Cr, (f) Fe, (g) Ni, (h) Co, (i) Cu, (j) Mn, (k) Mo, and (l) Si. 
A diffusion couple of TRIGA fuel versus 304SS was assembled and annealed at $1000^{\circ} \mathrm{C}$ for 24 hours. This couple developed a consistent and continuous interaction region (not shown) that extended through the width of the interaction interface, except for the edges. It had a thickness of approximately $15 \mu \mathrm{m}$. The presence of an interaction region verifies that the couple bonded. This diffusion couple, like the one annealed for 5 hours, lost bonding between the fuel and 304SS.

The interaction region resembles that of the diffusion couple of the same composition annealed at $1000^{\circ} \mathrm{C}$ for 5 hours. Several phase regions can be identified within the interaction region. The BE micrograph in Figure 52(a) shows that near the interaction region, the fuel also developed voids within the fuel microstructure near the interaction interface. The BE micrograph in Figure 52(b) details the interaction region.
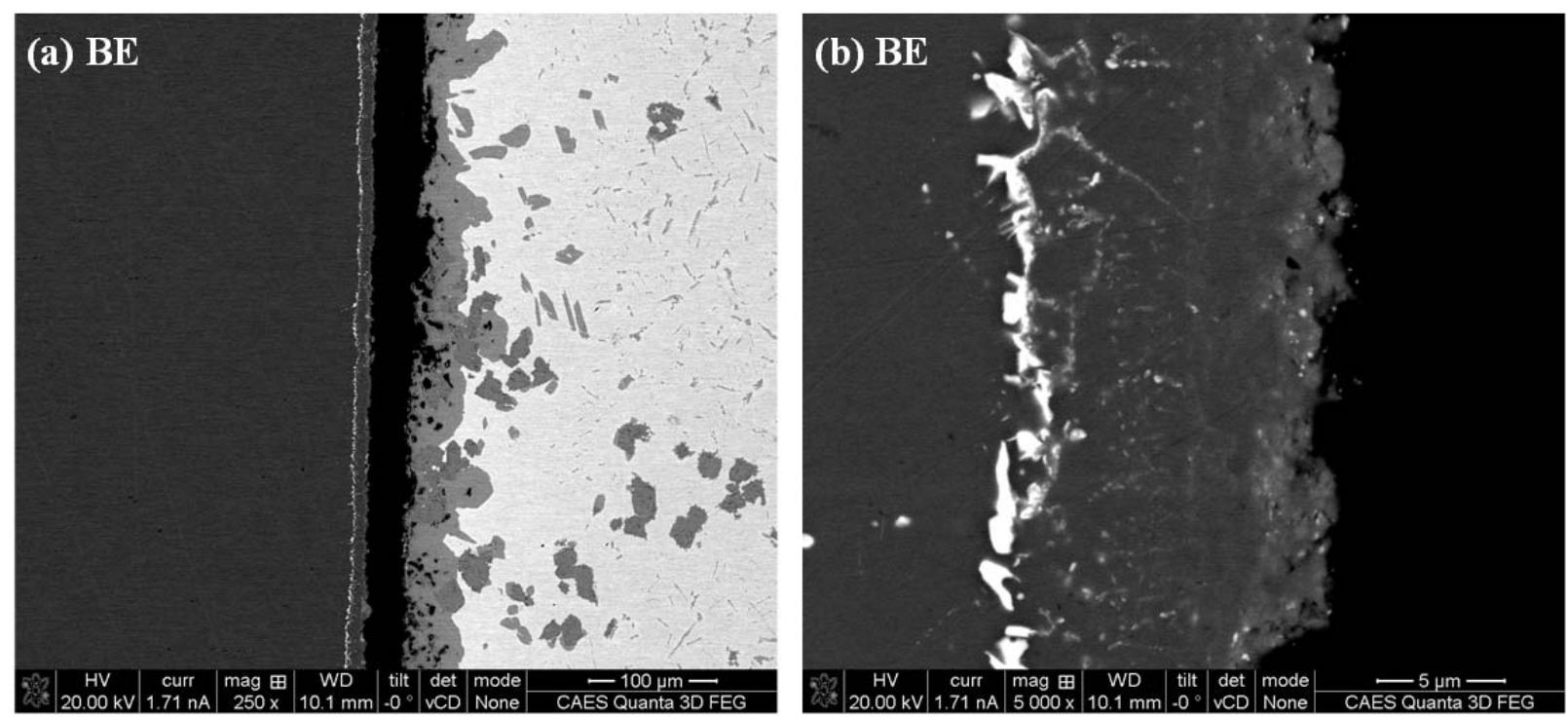

Figure 52. Backscatter electron micrographs of (a) a selected region from the TRIGA fuel versus 304SS diffusion couple annealed at $1000^{\circ} \mathrm{C}$ for 24 hours, and (b) a detailed micrograph of the interaction region.

EDS analysis was carried out in a selected region of the interaction region, as shown in the BE micrograph in Figure 53(a). Figure 53(b) through (e) show the respective qualitative EDS patterns of the different phase regions identified by EDS. Table 6 displays the results of the quantitative analysis of these phase regions. Comparison of the EDS patterns and quantitative data from this sample with those from the diffusion couple annealed for 5 hours, shown in Figure 48 and Table 4, shows that the average compositions of similar phase regions of the interaction region in the diffusion couples annealed for 5 and 24 hours are very similar. A difference in compositions between PR-4 and PR-4B can be seen from Table 6. This difference may arise because these PRs are composed of multiple-phases where the volumes of Zr-rich phases may vary within the selected areas where EDS was performed. The region selected for EDS analysis of the diffusion couple annealed for 24 hours did not include a phase region similar to the PR-5 found in the couple annealed for 5 hours. However, examination of Figure 52(a) indicates that this PR may be present in the microstructure. 

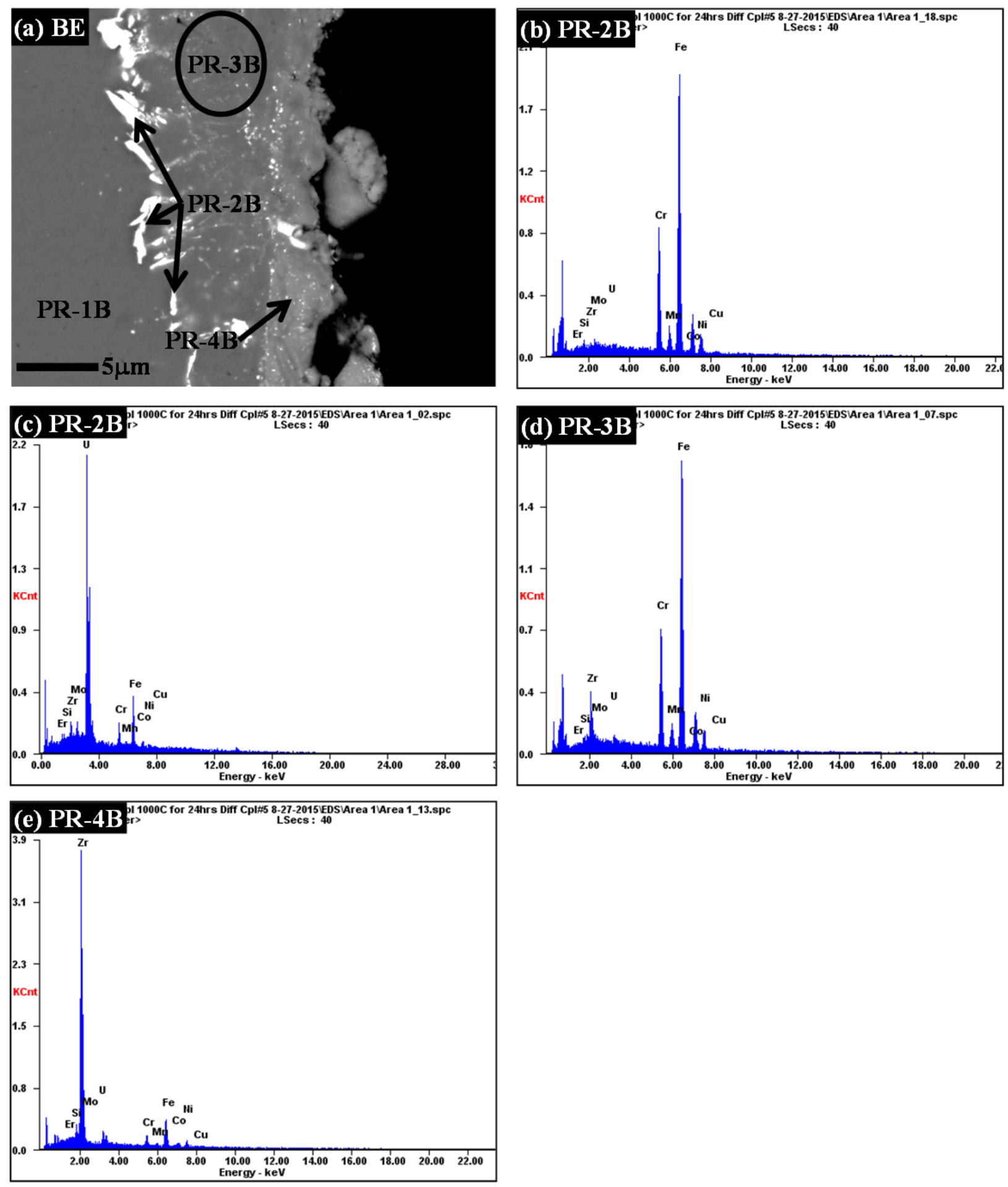

Figure 53. (a) Backscatter electron micrograph of the interaction region, in the diffusion couple annealed at $1000^{\circ} \mathrm{C}$ for 24 hours, tentatively showing several phases. (b) through (e) show typical EDS patterns for the differing phase regions (PR) in the interaction region where (b) PR-1B is the 304SS away from the interaction region, (c) PR-2B is a U-Fe-Cr-Ni-rich phase in interaction region, (d) PR-3B is Fe-Cr-Ni-rich phase, and (e) PR-4B is Zr-Fe-Cr-Ni-rich phase region. 
Table 6. Measured concentrations of the different phase regions observed in the interaction region of the diffusion couple annealed at $1000^{\circ} \mathrm{C}$ for 5 hours, as labeled in Figure 53.

\begin{tabular}{|c|c|c|c|c|}
\hline \multirow{2}{*}{ Element } & PR-1B & PR-2B & PR-3B & PR-4B \\
\cline { 2 - 5 } & \multicolumn{4}{|c|}{ Concentration (at.\%) } \\
\hline $\mathrm{Er}$ & $0.5 \pm 0.2$ & $2.7 \pm 1.2$ & $0.6 \pm 0.2$ & $0.9 \pm 0.6$ \\
\hline $\mathrm{U}$ & $0.2 \pm 0.1$ & $32.2 \pm 16.1$ & $0.4 \pm 0.1$ & $1.3 \pm 0.8$ \\
\hline $\mathrm{Zr}$ & $0.4 \pm 0.1$ & $3.6 \pm 2.4$ & $3.5 \pm 1.9$ & $49.1 \pm 17.6$ \\
\hline $\mathrm{Cr}$ & $19.2 \pm 0.2$ & $11.8 \pm 3.9$ & $18.7 \pm 0.3$ & $9.1 \pm 4.6$ \\
\hline $\mathrm{Fe}$ & $67.9 \pm 0.3$ & $35.8 \pm 13.8$ & $65.2 \pm 1.4$ & $30.6 \pm 14.1$ \\
\hline $\mathrm{Ni}$ & $7.2 \pm 0.2$ & $4.4 \pm 1.0$ & $6.8 \pm 0.4$ & $5.2 \pm 1.2$ \\
\hline $\mathrm{Co}$ & $0.4 \pm 0.1$ & $1.3 \pm 0.4$ & $0.4 \pm 0.2$ & $0.8 \pm 0.3$ \\
\hline $\mathrm{Cu}$ & $0.8 \pm 0.1$ & $1.3 \pm 0.4$ & $0.7 \pm 0.2$ & $1.0 \pm 0.2$ \\
\hline $\mathrm{Mn}$ & $1.8 \pm 0.1$ & $1.8 \pm 0.6$ & $1.9 \pm 0.2$ & $1.1 \pm 0.3$ \\
\hline $\mathrm{Mo}$ & $0.6 \pm 0.1$ & $3.0 \pm 4.2$ & $0.6 \pm 0.2$ & $0.5 \pm 0.2$ \\
\hline $\mathrm{Si}$ & $1.1 \pm 0.2$ & $1.9 \pm 0.8$ & $1.2 \pm 0.3$ & $0.4 \pm 0.3$ \\
\hline
\end{tabular}

Similarly, to the fuel annealed for 5 hours, x-ray maps were generated from a selected section of the interaction region to identify the presence and location of the elements from the fuel and the 304SS. The $\mathrm{x}$-ray maps are in good agreement with the EDS analysis performed in a similar section of the interaction region. This analysis revealed a small section within the interaction region that was enriched with Er and Co. This composition was not identified in any EDS analysis.

Comparison of the x-ray maps collected from the interaction region of the diffusion couple annealed for 5 hours with those collected for the 24-hour anneal indicates that both interaction regions exhibited similar phase development. Longer annealing, from 5 to 24 hours, did not show significant variations. The similarities may have arisen from failure of the bond at the interaction interface between the 304SS and fuel. 

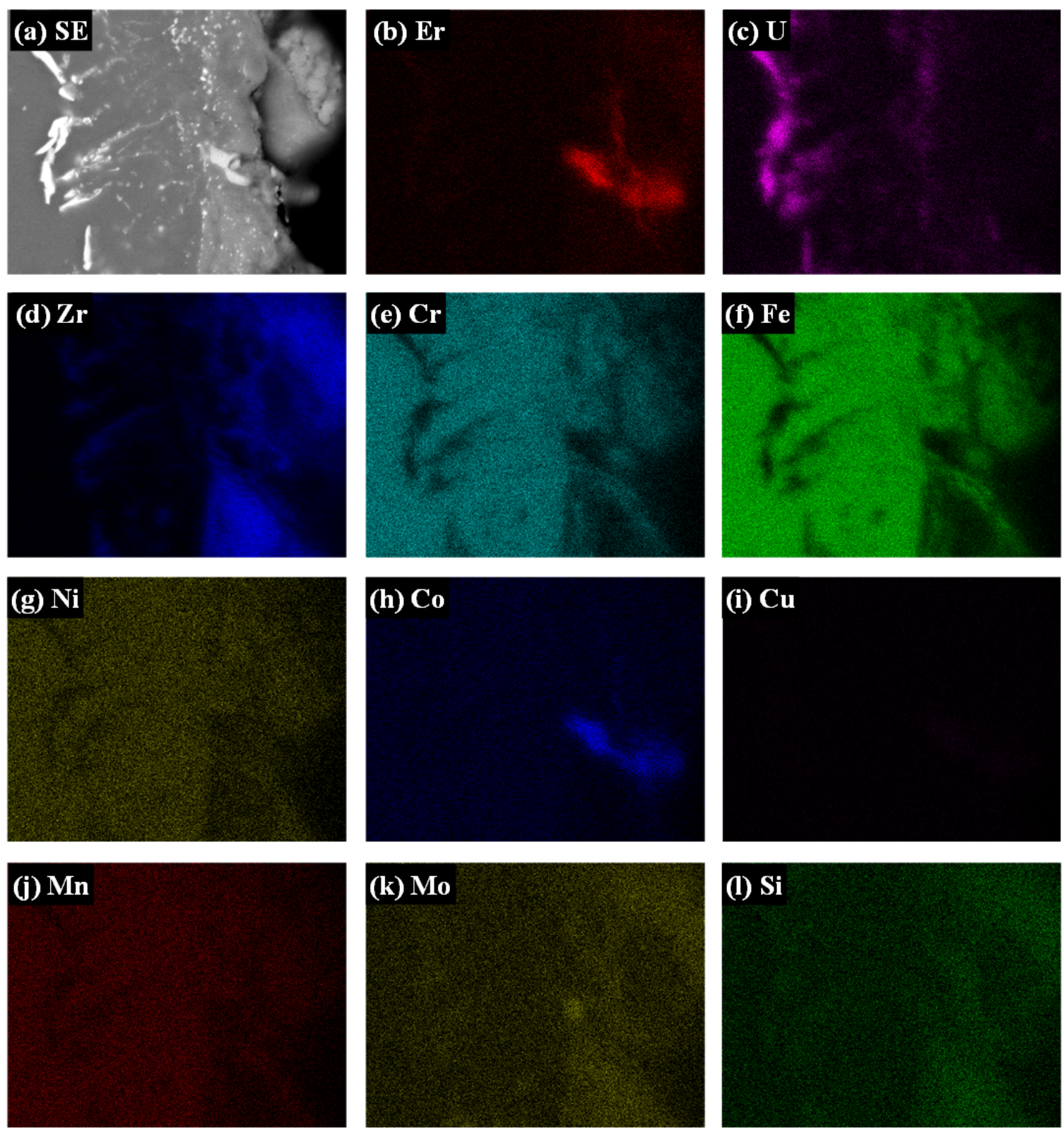

Figure 54. (a) SE micrograph of the interaction region in the diffusion couple annealed at the $1000^{\circ} \mathrm{C}$ for 24 hours, and x-ray maps of the region qualitatively showing the presence and location of (b) Er, (c) U, (d) $\mathrm{Zr}$, (e) Cr, (f) Fe, (g) Ni, (h) Co, (i) Cu, (j) Mn, (k) Mo, and (l) Si.

The time of failure of the bonding interface is not known for either diffusion couple annealed at $1000^{\circ} \mathrm{C}$. However, the thickness of the interaction regions of the diffusion couples annealed for 5 and 24 hours were nearly identical. This result is not consistent with diffusion couples that remained bonded through an annealing process at constant temperature with varying annealing time. The nearly identical thicknesses strongly suggest that the interaction-interface bond in each diffusion couple failed at the same, or nearly the same, time during annealing. A likely explanation for this failure is shrinking of the TRIGA fuel during annealing as a result of hydrogen diffusion out of the fuel. Because the volume of the fuel can decrease significantly, stress would build at the bonding interface, eventually resulting on fracturing. An experiment was carried out to determine if the fuel would indeed experience significant loss of volume during annealing at $1000^{\circ} \mathrm{C}$. The details and results from this experiment are described in Section 3.3 below. 
Because the diffusion couples developed interaction regions with similar thickness after significantly differing annealing times, the evolution of the interaction region as a function of time could not be investigated. As a result, the data required (diffusion coefficients) to calculate the time that elements from the fuel $(\mathrm{U}, \mathrm{Zr}, \mathrm{Er})$ would require to penetrate the cladding material at high temperature in cladded-reactor fuels could not be acquired. Diffusion couples that remain bonded through the annealing process are required. Thus, this data remains a source for future research.

\subsubsection{Characterization by Transmission Electron Microscopy}

To identify the phases in the interaction regions of the TRIGA fuel versus 304SS diffusion couples, TEM characterization was performed on the interaction region of the diffusion couple annealed at $1000^{\circ} \mathrm{C}$ for 5 hours. The sample was prepared via a FIB in situ lift-out technique. The sample was selected, based on the SEM analysis, from a region that encompassed all or most of the phases observed within the microstructure. The HAADF micrograph in Figure 55 shows the prepared TEM sample, and comparison with Figure 47 shows that the TEM sample captured the complete thickness of the interaction region.

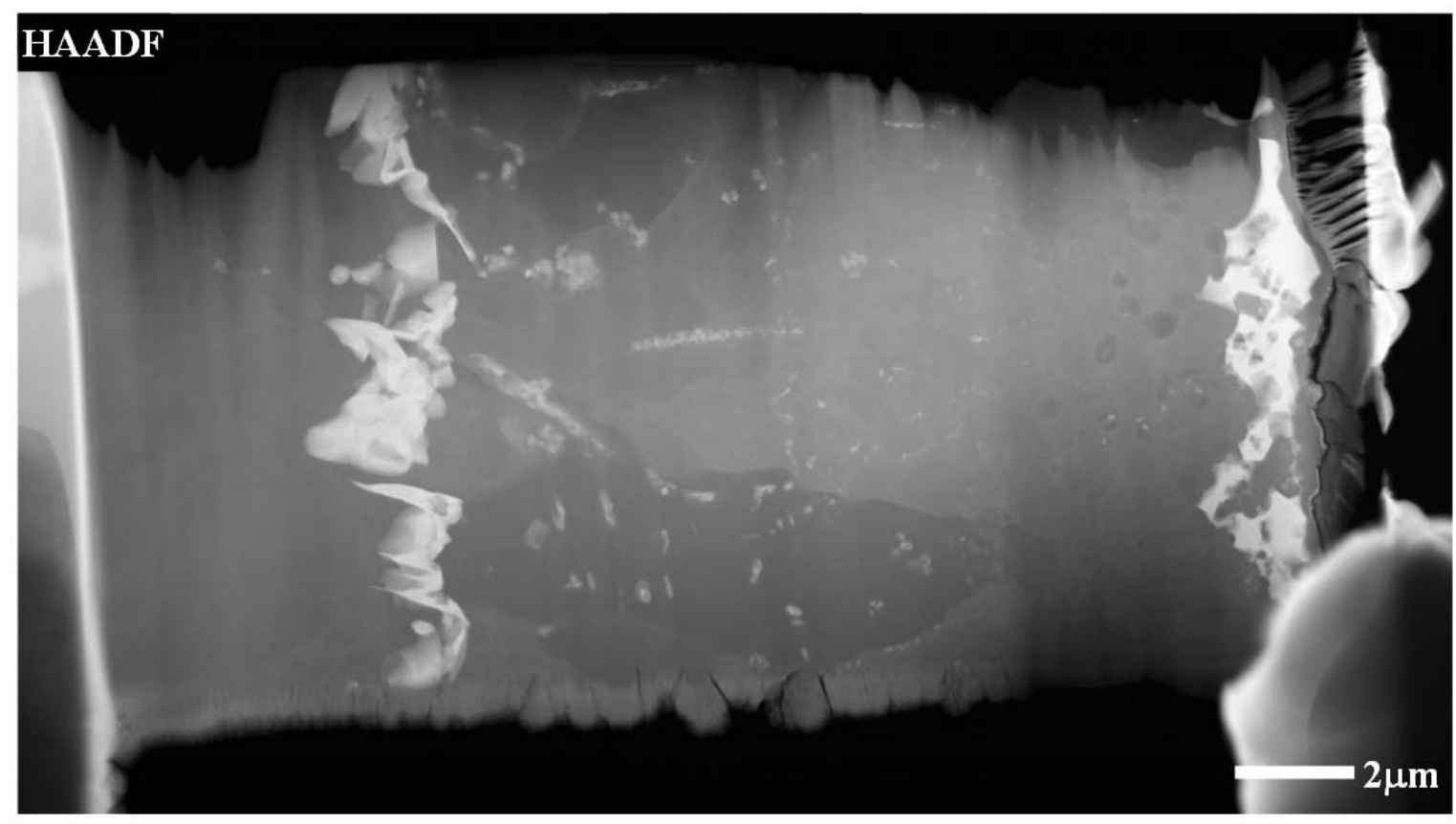

Figure 55. HAADF micrograph of the TEM sample obtained from the interaction region of the diffusion couple of TRIGA fuel versus $304 \mathrm{SS}$ annealed at $1000^{\circ} \mathrm{C}$ for 5 hours. The sample encompasses the entire thickness of the interaction region.

Characterization by TEM was performed to carry out a more-detailed analysis than that possible by SEM. EDS was performed in the TEM on specific grains to obtain their compositions. Special interest was paid to phases containing U-Fe compositions to identify low-melting temperature phases. 
The HAADF micrograph in Figure 56 details the region that contained the U-Fe-Cr-Ni enriched phase region that was identified in the SEM analysis as PR-2 in the BE micrograph in Figure 48. The EDS patterns in Figure 56(b) through (f) suggest that this phase region contained more than a single phase. The pattern in Figure 56(b) identified a grain with $U$ that contained a significant $C$ concentration. In order of decreasing U-concentration from Figure 56(b) through (f), a significant decrease in $\mathrm{U}$-concentration was observed, marked by increases in $\mathrm{Fe}, \mathrm{Cr}$, and $\mathrm{Ni}$ concentrations. The patterns strongly suggest that U-rich and Fe-rich phases may be present in this region.

Figure 57 details the one section of the elongated apparent phases within PR-3 observed in Figure 48 between PR-2 and PR-4. These apparent phases were too small for accurate EDS analysis in the SEM. The specific region under analysis in Figure 57(a) is marked by a square in the HAADF micrograph in Figure 56(a). The grains within this region generally contained U-Zr-Fe-Cr-Ni compositions. A grain was identified that contained significant Mo-concentration as shown in Figure 57(c). Another grain, labeled (e) was identified to contain a Mo-U-Zr-Fe-Cr-Ni phase with low Mo, U, and Zr concentrations. Based on phase contrast, the region labeled as (g) contained multiple very-fine-grained phases. The EDS pattern in Figure 57(e) shows a measurable Mo-concentration. Likely, at least one of the phases in (g) was

Mo-enriched. Analysis carried out on a similar region, shown in Figure 58, also indicates the presence of Mo within the grains of the elongated phase regions. The HAADF micrographs indicate that the apparent elongated phases are composed of grains with differing compositions, likely different phases, and can be identified as a distinct phase region within PR-3. 

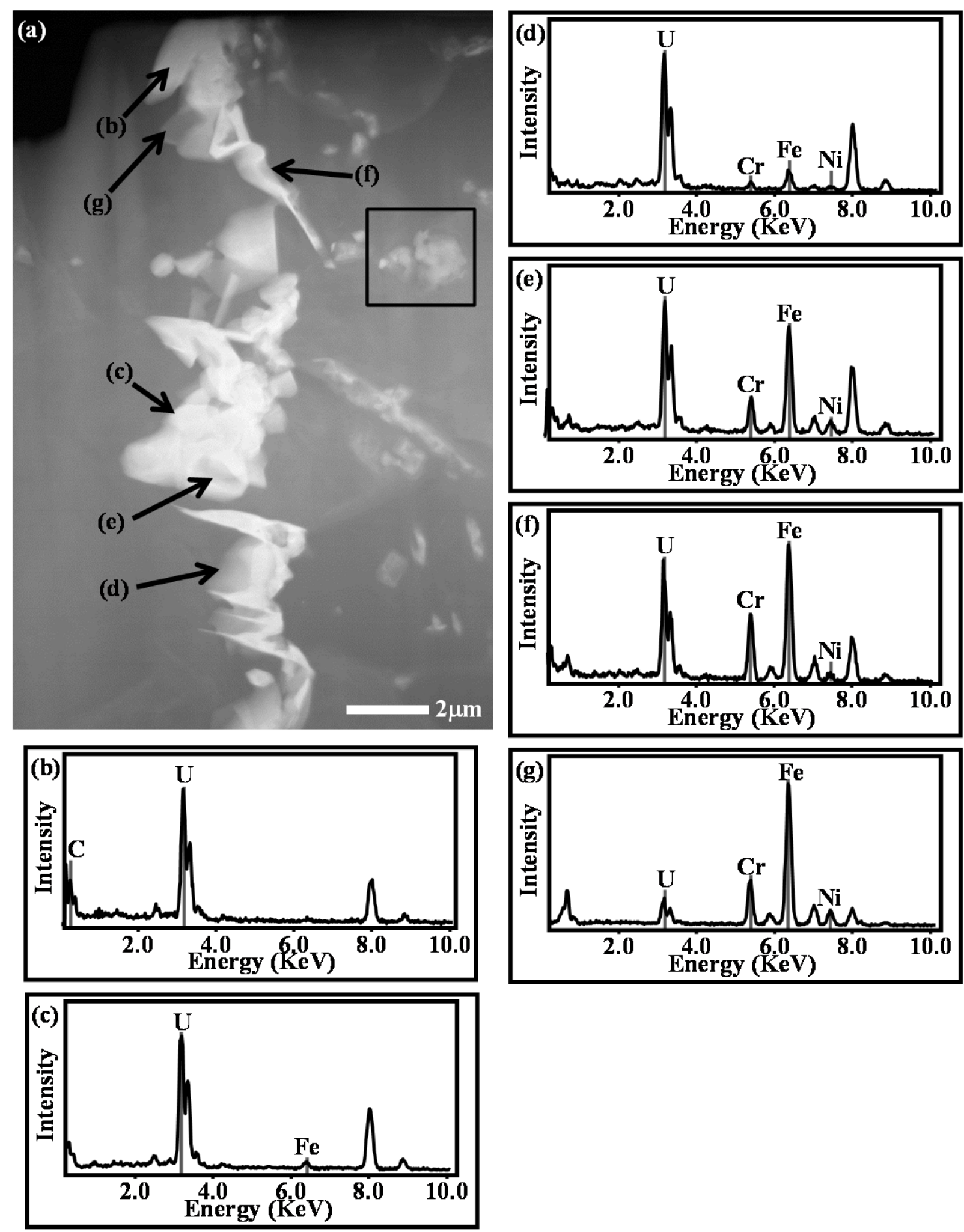

Figure 56. (a) Selected area of the interaction region showing the U-Fe-Cr-Ni-rich phase region (PR-2) in the interaction region. The EDS patterns in (b) through (f) show that the grains within this phase region contain distinct compositions with significant variations in $\mathrm{C}, \mathrm{Cr}, \mathrm{Fe}$, and $\mathrm{U}$. 

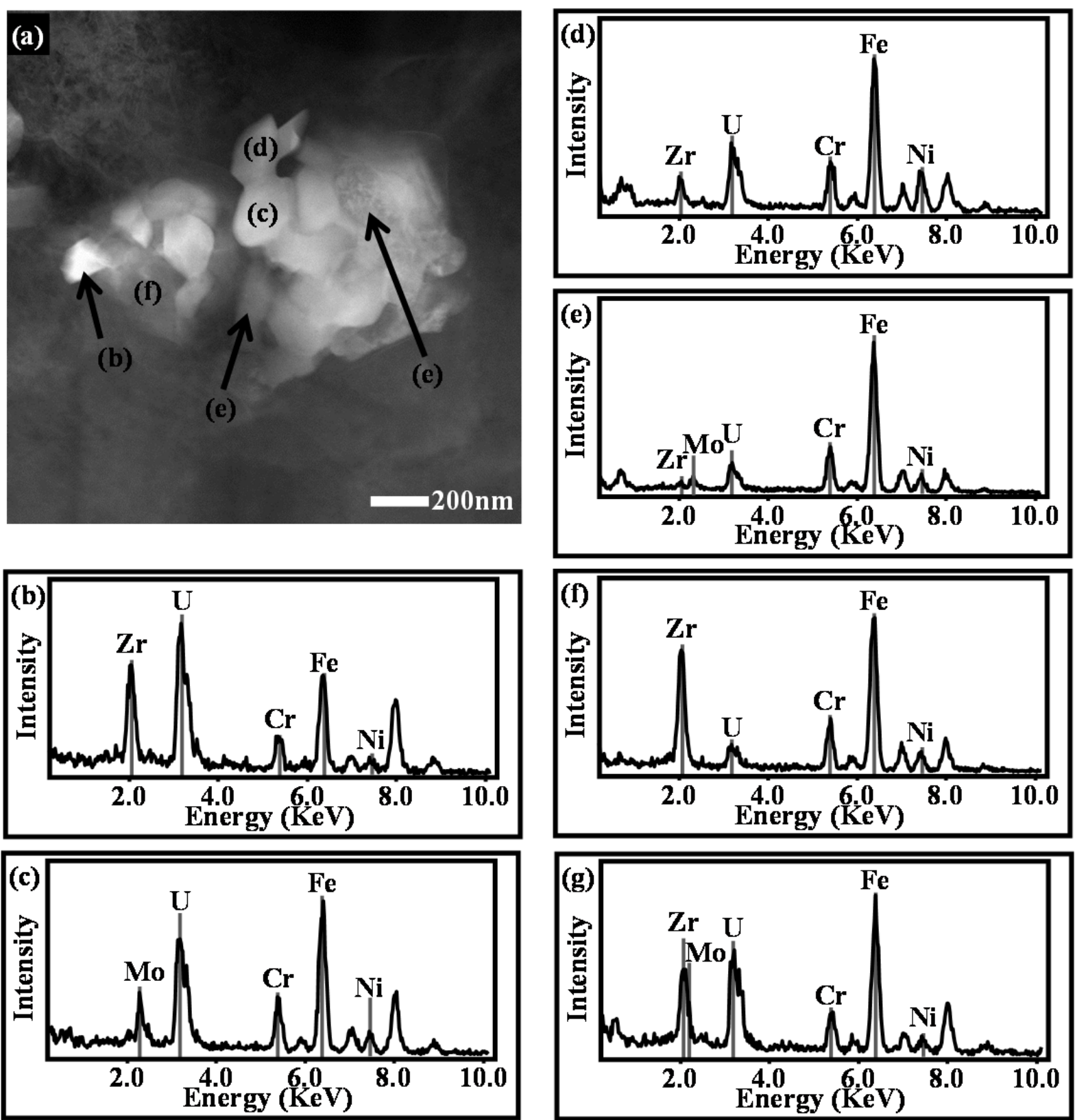

Figure 57. (a) Detailed micrograph of the elongated phase regions observed in the interaction region microstructure. The micrograph shows details from the region marked by a square in Figure 56 .

(b) through (g) show the differences in compositions of the specific grains labeled in (a). 

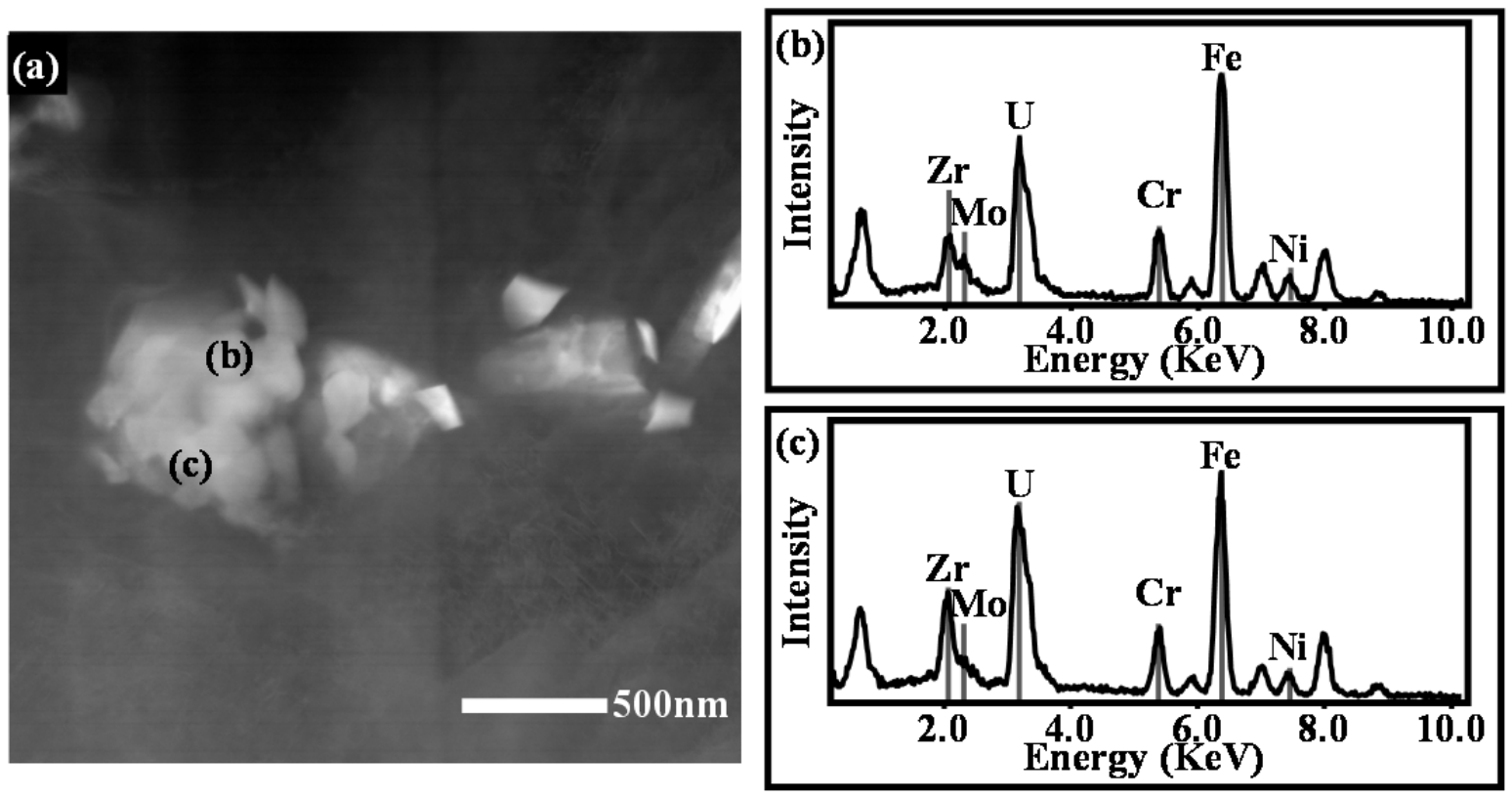

Figure 58. (a) Detailed micrograph of the elongated phase regions observed in the interaction region microstructure. The EDS patterns in (b) and (c) show similar compositions to the EDS analysis in Figure 57, and show measurable Mo-concentrations.

The U-Zr-Fe-Cr-Ni-rich phase region very near the interaction interface between the TRIGA fuel and 304SS, identified as PR-5 in Figure 48, is detailed in the HAADF micrograph in Figure 59(a). This micrograph, along with the EDS patterns in Figure 59(b) through (f), confirms the composition measured by the EDS analysis in the SEM. However, the region, based on phase contrast and significant difference in compositions, appears to contain multiple phases. U-Fe-Cr-Ni-rich, U-Zr-Fe-Cr-Ni-rich, and $\mathrm{Zr}-\mathrm{Fe}-\mathrm{Cr}-\mathrm{Ni}$-rich phases may be present in this region. A thin layer containing only Zr, according to the pattern in Figure 59(f), appears to separate the U-Zr-Fe-Cr-Ni-rich phase region (PR-5) from the fuel/304SS interaction interface. Comparison of the HAADF micrograph in Figure 59 with the BE micrograph in Figure 47(b) and Figure 48(a) suggests that the U-Zr-Fe-Cr-Ni-rich phase region and the $\mathrm{Zr}$ thin layer may be a section of the TRIGA fuel that remained attached to the 304SS when the interaction interface debonded during annealing. If so, then the possibility exists that after failure of the interaction interface, the TRIGA fuel piece could have continued to interact with the 304SS to develop this observed phase region.

Figure 60 details the region, near the U-Zr-Fe-Cr-Ni-rich phase region from Figure 59(a) that corresponds to PR-4 in Figure 48. Figure 60(a) shows a contrast enhanced HAADF micrograph that shows darker contrast grains, labeled (b) and (c) within the interaction region. The EDS patterns in Figure 60(b) shows grains containing only $\mathrm{Zr}$ (may also contain hydrogen). Figure 60(c) shows $\mathrm{Zr}-\mathrm{Fe}-\mathrm{Cr}-\mathrm{Ni}$ grains. The EDS pattern in Figure 60(d) shows Fr-Cr-Ni grains. These results support the interpretation of the EDS patterns in Figure 48(e) and indicate that PR-4 contains two or three distinct phases. 

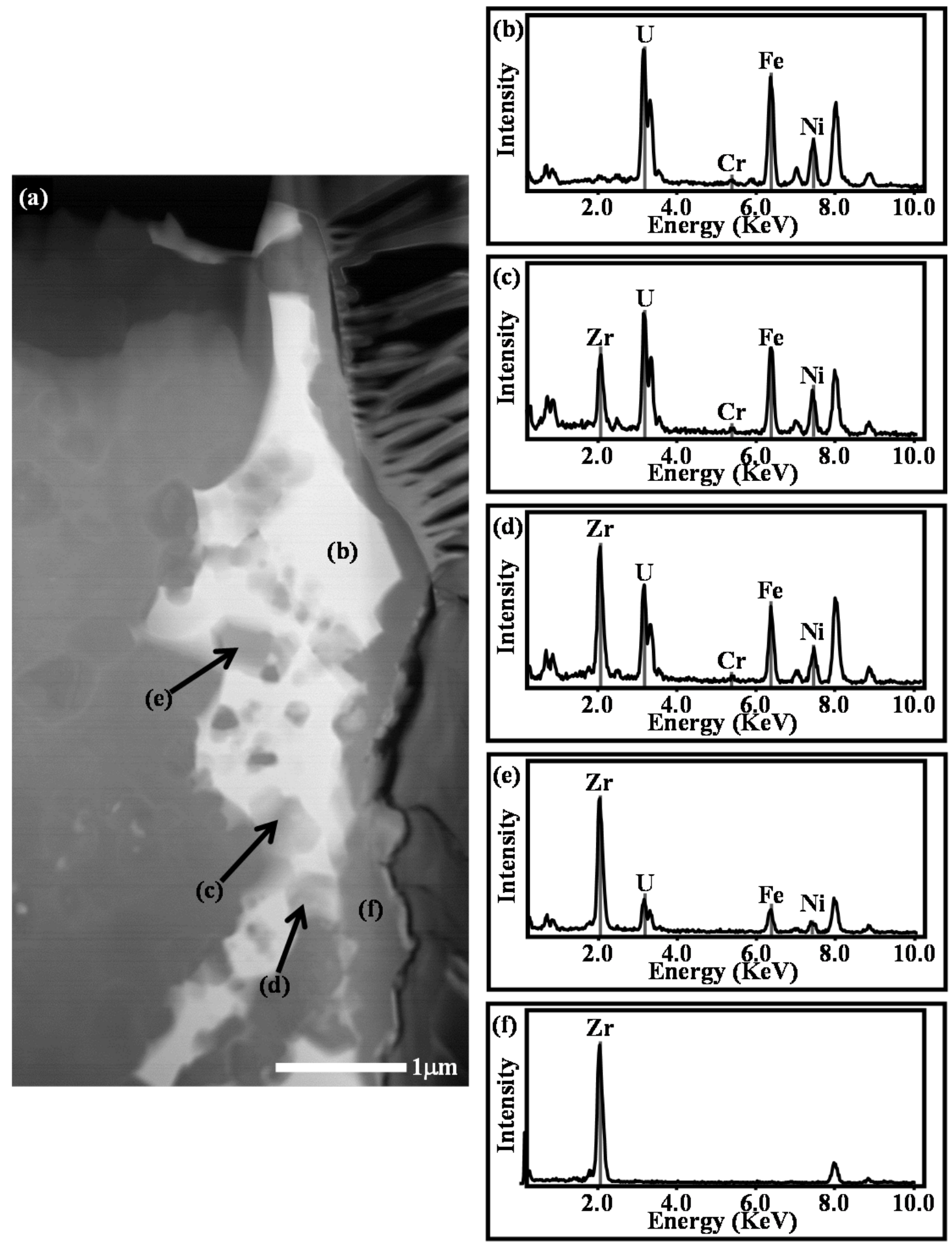

Figure 59. (a) Selected area of the interaction region showing the U-Zr-Fe-Cr-Ni-rich phase region (PR-5) in the interaction region. The EDS patterns in (b) through (f) show that the grains within this phase region contain distinct compositions with significant variations in $\mathrm{Cr}$, $\mathrm{Fe}, \mathrm{U}$, and $\mathrm{Zr}$. 


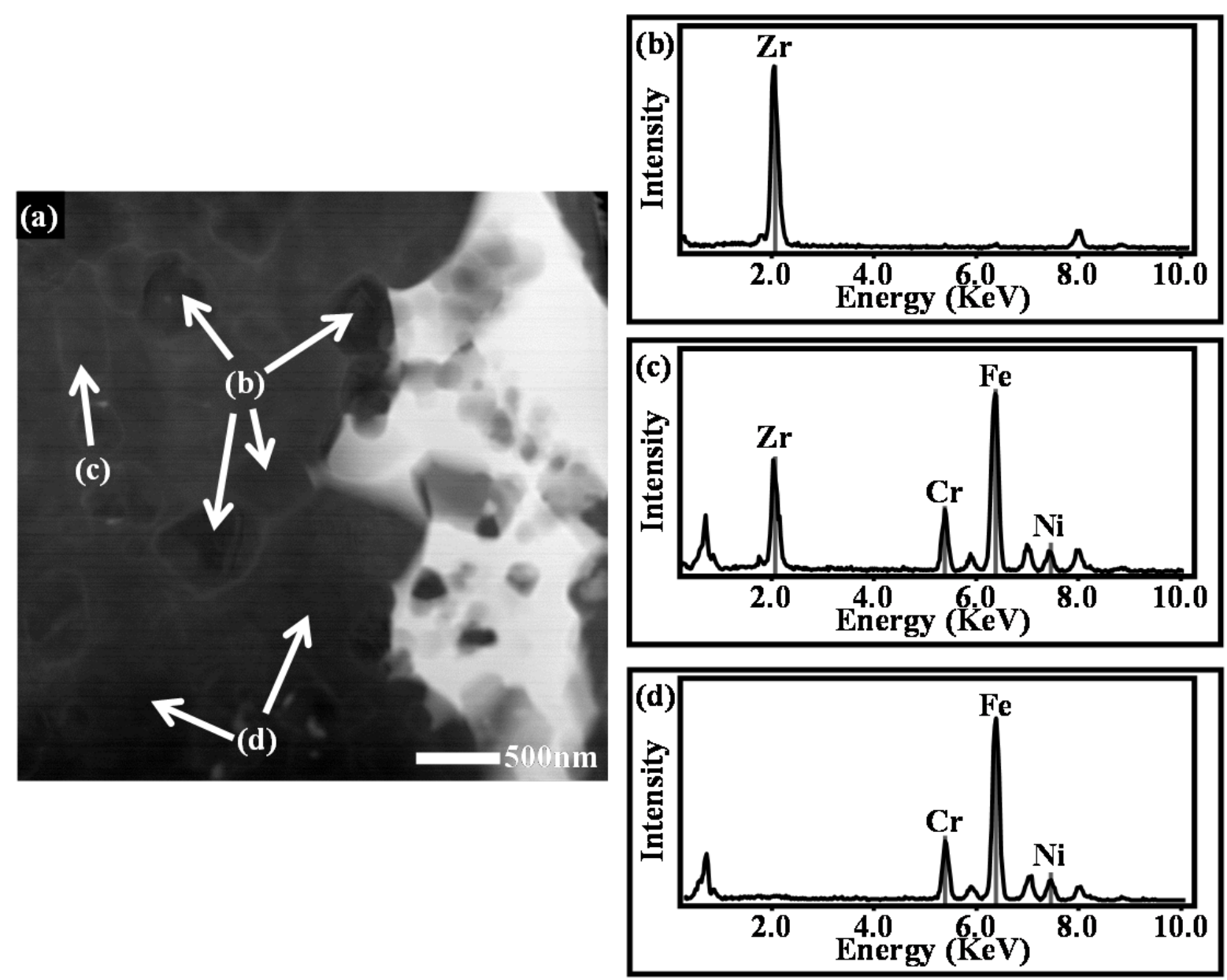

Figure 60. (a) Detailed micrograph of the Zr-Fe-Cr-Ni-rich phase region (PR-4) near the interaction interface with TRIGA fuel. (b), (c) and (d) EDS patterns showing the grains with only Zr and $\mathrm{Zr}-\mathrm{Fe}-\mathrm{Cr}-\mathrm{Ni}$, and Fe-Cr-Ni compositions, respectively.

Diffraction analysis was carried out on selected grains of the interaction region to obtain crystallographic data to identify the specific phases within the interaction region. Based on the U-Fe binary phase diagram, emphasis was given to phases containing $\mathrm{U}$ and $\mathrm{Fe}$ to attempt to positively identify low-melting-point phases.

Grains were selected for crystallographic analysis based on the results from the characterization by EDS. Typically the interaction region grains contained several of the elements found in both the fuel and 304SS. Because crystallographic data of multi-component phases ( $>2$ elements) is not readily available for most possible phases in this system, in most cases, characterization of the collected diffraction patterns assumed that the phases analyzed fitted binary-alloy phases.

As in previous analysis, because the presence of hydrogen could not be determined by EDS, the analysis assumed it to be present. If a hydride phase was found to fit the diffraction patterns, the grain under analysis was tentatively characterized as a hydride. If the non-hydride phase with the same composition, as determined by EDS, was found not to fit the patterns, then the grain was characterized as a hydride. 
The nomenclature used in Figure 48 will be used in this section to identify the location of the diffraction analysis. The BF micrograph in Figure 61(a) shows a grain selected from PR-2 for diffraction analysis. EDS analysis of this grain is shown in Figure 56(b). Based on EDS, this grain contained only U and C. Diffraction analysis, shown in Figure 61(b) through (d) confirmed that the grain is composed of the cubic-UC phase (cF8, SG:Fm-3m).
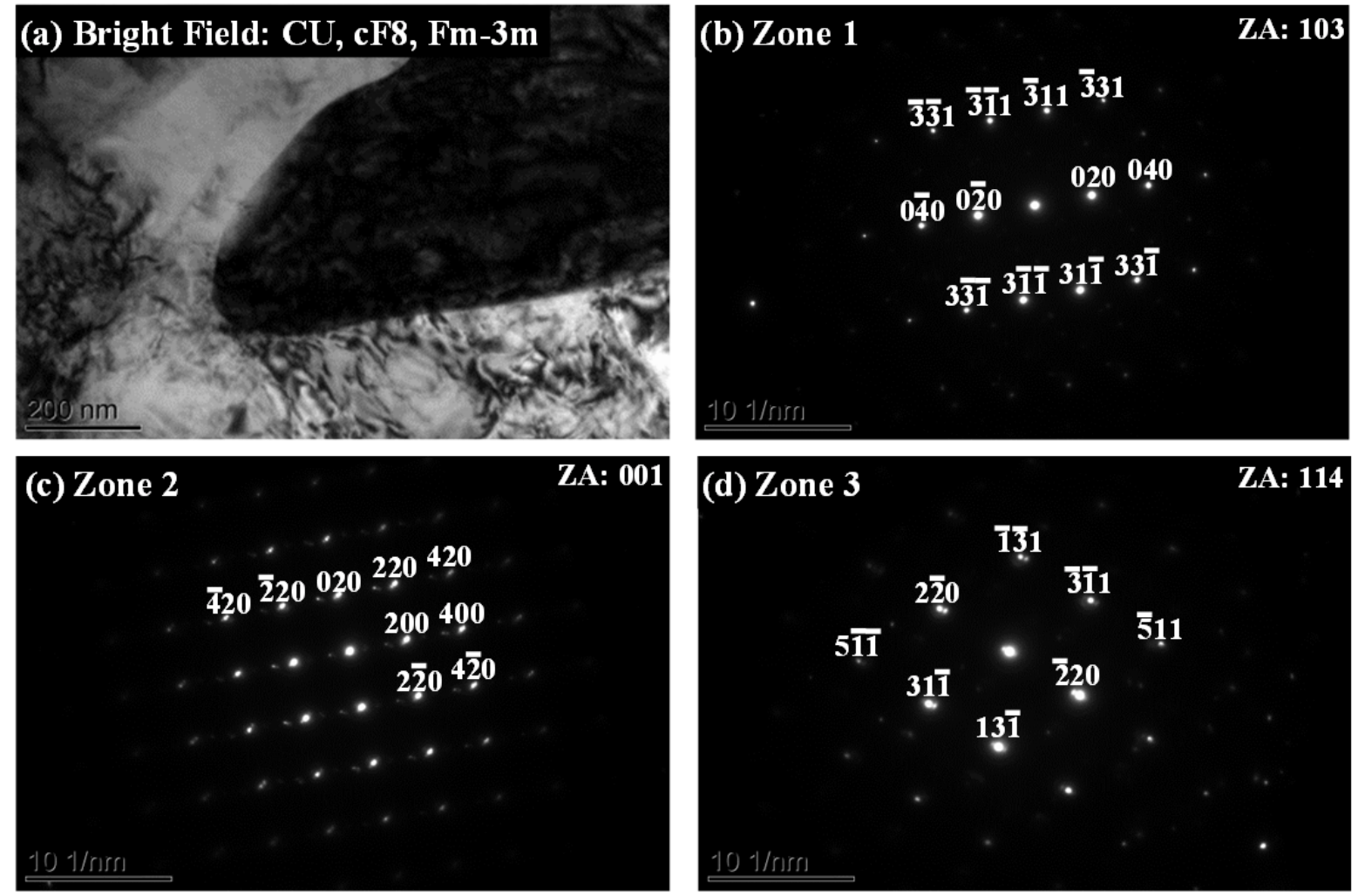

Figure 61. (a) Diffraction analysis of a selected grain within PR-2 in the interaction region. The diffraction patterns in (b) though (d) identify the grain as the cubic-UC phase.

The BF micrograph in Figure 62 shows a different grain selected from PR-2. EDS from this grain is shown in Figure 56(g). EDS indicated that this grain contained $\mathrm{U}$ in low concentration and $\mathrm{Fe}$ in high concentration, with significant concentrations of $\mathrm{Cr}$ and Ni. Diffraction analysis identified this grain as containing the $\gamma$-Fe phase (cF4, SG:Fm-3m), the typical phase for the austenitic 304SS. The diffraction data combined with EDS indicate that 304SS has some solubility for U. 

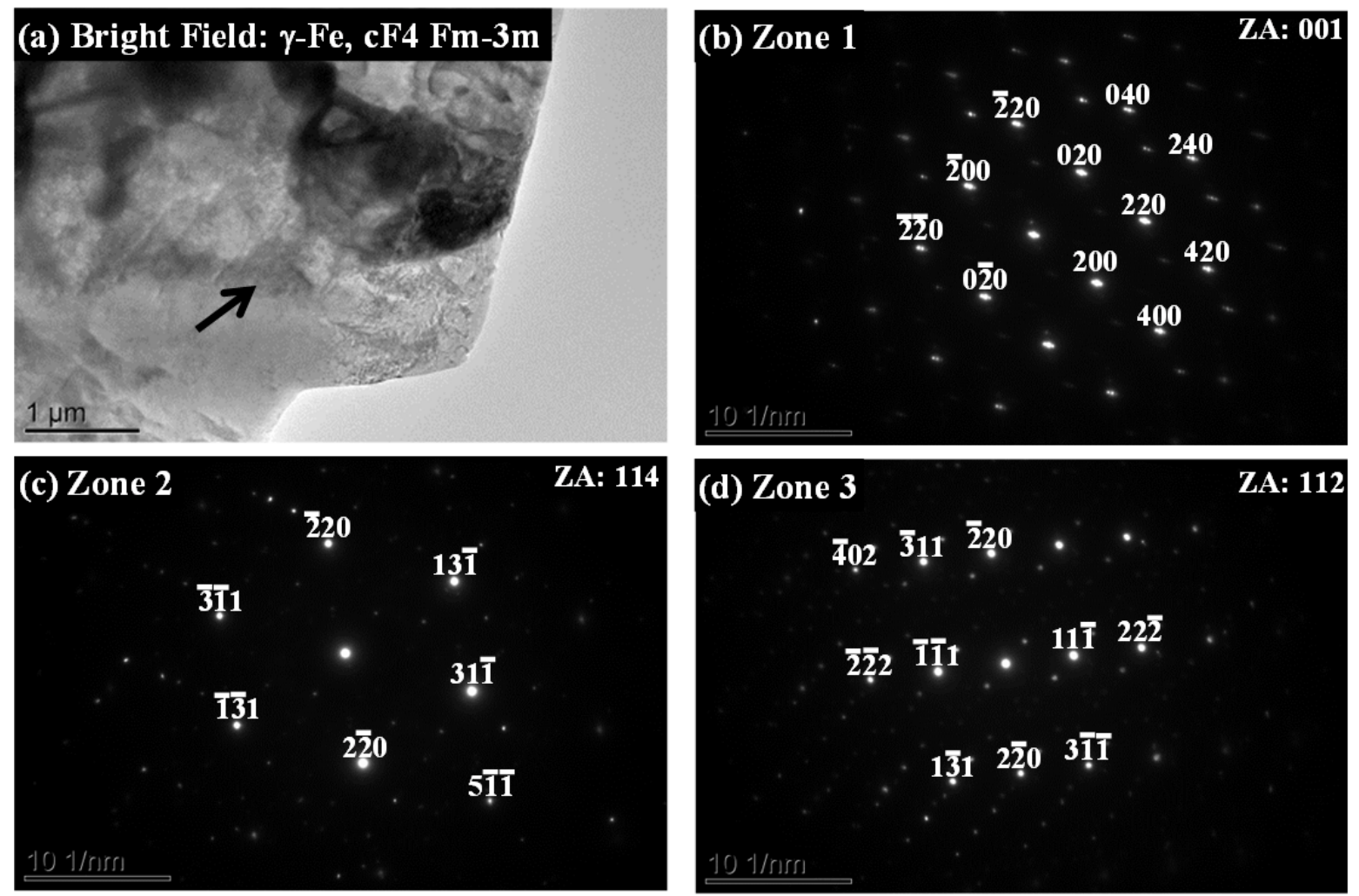

Figure 62. (a) Diffraction analysis of a selected grain within PR-2 of the interaction region. The diffraction patterns in (b) though (d) identify the grain as the $\gamma$-Fe phase.

The BF micrograph in Figure 63(a) was selected from PR-5 in Figure 48. Its composition was shown in Figure 59(b), and it shows that this grain contained significant concentrations of $U$ and Fe with lower $\mathrm{Cr}$ and Ni concentrations. Diffraction analysis identified the $\mathrm{Fe}_{2} \mathrm{U}$ phase (cF24, SG:Ff-3m). This analysis reveals, that at least one of the binary phases that result in the low temperature eutectic composition in the $\mathrm{Fe}-\mathrm{U}$ phase diagram can develop in the interaction region between TRIGA fuels in contact with 304SS claddings. 

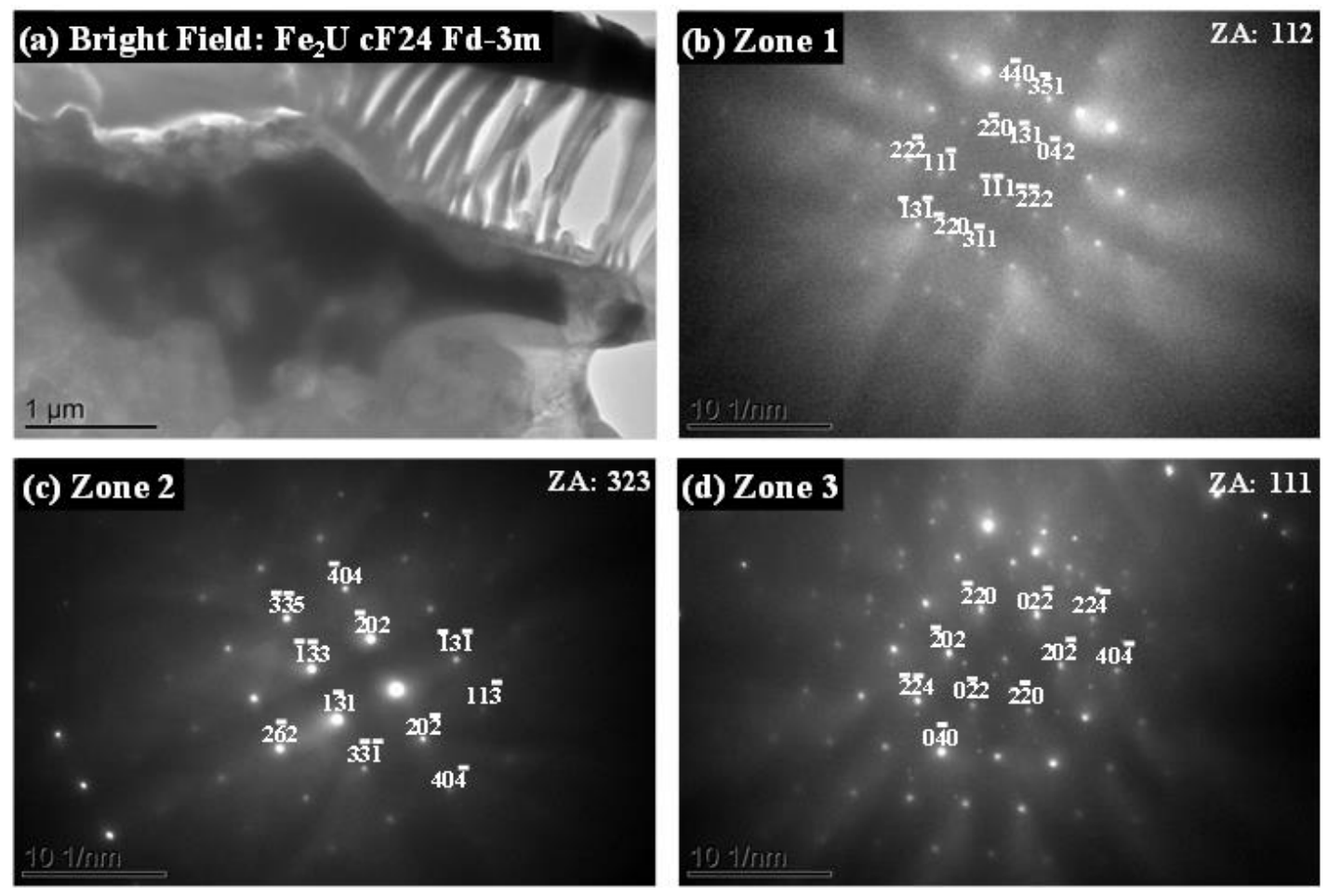

Figure 63. (a) Bright field micrograph of a grain selected from PR-5. EDS for the grain, shown in Figure 59(b) indicates that this grain contained significant concentrations of $\mathrm{U}, \mathrm{Fe}$, and Ni. Diffraction analysis, shown in (b) through (d), show that this grain was composed of the cubic- $\mathrm{Fe}_{2} \mathrm{U}$ phase.

The U-Fe binary phase diagram, shown in Figure 64, indicates that the $\mathrm{Fe}_{2} \mathrm{U}$ is a phase that can develop as a result of eutectic reactions from the liquid phase. The developed solid would likely be composed of a two-phase system that would depend on the overall composition of the system. The overall composition of the interaction region in the diffusion couple annealed at $1000^{\circ} \mathrm{C}$ for 5 hours has not been accurately measured due to the inability to detect hydrogen. The elements additions of the fuel and the 304SS, such as $\mathrm{Zr}, \mathrm{H}, \mathrm{Cr}, \mathrm{Ni}$, and the minor element additions, result in increased complexity due to an increase the thermodynamic variables in the system.

Identification of a two-phase region containing $\mathrm{Fe}_{2} \mathrm{U}$ and $\mathrm{FeU}_{6}$ phases within the interaction region of the diffusion couple would strongly suggest that the eutectic reaction at approximately 67 at.\% $\mathrm{U}$ in the binary phase diagram had taken place in the diffusion couple. Characterization of the interaction region identified the $\mathrm{Fe}_{2} \mathrm{U}$ phase. Characterization of the interaction region by EDS in the TEM, in Figure 50 and Figure 56 through Figure 59, shows that phases containing high concentrations of $U$ and Fe may be present within the microstructure, which strongly suggests that a phase containing the $\mathrm{FeU}_{6}$ composition may be present in the sample. As of this writing, full characterization of the interaction region has not been completed. Many of the high U-Fe phases have not been characterized by crystallographic analysis to identify the phase. Further analysis is required. 


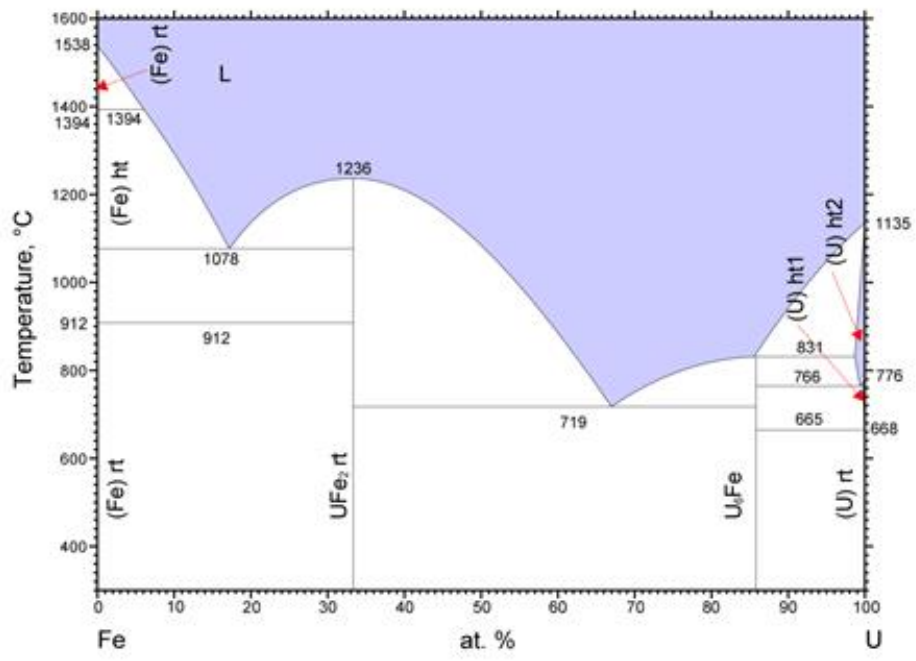

O ASM International 2006. Diagram No. 2002172.

Figure 64. U-Fe binary phase diagram.

\subsection{Fuel Shrinkage Experiment}

The interaction region in the couple annealed at $1000^{\circ} \mathrm{C}$ for 5 hours is approximately the same thickness as that of the couple annealed at $1000^{\circ} \mathrm{C}$ for 24 hours. The interaction region in the couple annealed for 24 was expected to be significantly thicker. The similarity in thickness indicated that during annealing the bonding interfaces failed at similar time intervals. Because characterization of the couples did not show any indication of oxidation of the interfaces, and because the couples bonded for a time interval to allow diffusion to take place, another mechanism was expected to be the cause of the failure of the interface. Shrinkage of the fuel was considered as a candidate.

To investigate this possibility, a simple experiment was carried out to determine if the TRIGA fuel experienced shrinkage during annealing. The 304SS was not tested, but shrinkage was not expected. A sample of the TRIGA fuel was annealed in the furnace at $1000^{\circ} \mathrm{C}$ for 24 hours. The sample was prepared and annealed in the same fashion as those used in the diffusion couple experiments, including wrapping the fuel in Ta to minimize oxidation.

The initial sample weight and dimensions were $0.7058 \mathrm{~g}$ and $2.98 \mathrm{~mm} \times 6.27 \mathrm{~mm} \times 5.10 \mathrm{~mm}$. After annealing the sample weight was $0.6987 \mathrm{~g}$, and the sample measured $2.84 \mathrm{~mm} \times 6.02 \mathrm{~mm} \times 4.86 \mathrm{~mm}$. The sample weight loss was $0.0071 \mathrm{~g}$, which was a $13 \%$ loss of its volume.

The results indicate that during annealing, enough shrinkage of the contact area and thickness of the fuel took place to fracture the interaction interface. The loss in thickness should have ensured that once separated, the surfaces would be unlikely to come in contact again in the jigs with the current design. The similar thickness of the interaction regions in the diffusion couples also suggest that both couples lost bonding prior to 5 hours of annealing. Future diffusion couple experiments need to take fuel shrinkage into account to ensure good contact of the interaction surfaces.

\section{FUTURE WORK}

Characterization of the TRIGA fuels in the as received and annealed condition identified several phases in the microstructures. Characterization by TEM was limited by time constraints, and several features observed within the microstructures were not fully identified. To develop a better understanding of the fuel evolution as a result to exposure to high temperature, characterization should be completed. 
The diffusion couples annealed at $1000^{\circ} \mathrm{C}$ revealed that complex interaction regions could develop as a result of interactions between TRIGA fuels and 304SS claddings. Characterization of the interaction region was carried out with an emphasis on identification of low-melting-point phases. Full characterization of the U-Fe phase regions present in the microstructure must be completed to determine whether the low-temperature eutectic phase composition develops.

Full characterization of the interaction regions to identify all the phases that develop in the interaction regions has not been the main goal of this project. However, due to the complexity of the interaction region, full characterization would is recommended to determine if any unexpected low-temperature phases, binary or multi-component, exist in the microstructure.

Diffusion couple experiments at lower temperatures may also be desired to study the phase development of the interaction region at temperatures more representative of loss-of-coolant-accident scenarios, where $950^{\circ} \mathrm{C}$ is the high temperature safety limit of the fuel [9]. Experiments at $800^{\circ} \mathrm{C}$ may also be desired to incorporate the experience gained in this study to replicate previous studies at this temperature where oxidation appears to have presented an obstacle.

Diffusion couple experiments of TRIGA fuel versus 304SS determined that the fuel bonds to the 304SS early in the annealing process. After some time, the couple fractures at the bonding interface due to the fuel shrinkage. This has prevented the determination of growth of the interaction region. As a result, the rate of penetration of the interaction products has not been measured. Further diffusion couple experiments should be carried out to precisely measure the growth rate of the interaction region. Methods to prevent failure of the bonding interface must be developed. One method can include spring loading of the couple so that the surfaces are under constant pressure during annealing. Simplified diffusion couples can be annealed with U-Zr alloys replacing the TRIGA fuel. Also, a sample of TRIGA fuel sample can be annealed in a furnace until sintering is complete and shrinkage stops. This sample can then be used in a diffusion couple. The optimal method must be determined and then utilized to prepare diffusion couples with consistent interaction regions.

Characterization of the fuel by TEM has been limited by the similarities of the diffraction patterns, and by lack of data on the precise hydrogen concentration in both the fuel and the interaction region in the diffusion couples. X-ray diffraction can be utilized to characterize the bulk fuel in the as-received and annealed conditions. X-ray diffraction can also be used to characterize the interaction region bulk phase compositions. Due to the nature of this analysis, the specific locations of any identified phase would not be precisely determined. However, x-ray diffraction would provide an invaluable complementary characterization method for the TEM analysis.

To quantify the presence and location of hydrogen in the systems under study, the Local Electrode Atom Probe may be employed to determine the precise atomic scale structure of the samples. This method presents some challenges for the analysis of hydrogen that must be considered prior to any characterization. Even so, this characterization method can provide data as to the effect of hydrogen in the fuel/cladding system and could provide invaluable data to the understanding of the phase development that result from interactions between TRIGA fuel elements and the 304SS claddings.

\section{REFERENCES}

1. Gruia, M., I. Ciucă, M. Abrudeanu, T. Meleg, I. Dumitrscu, 2013, "Evaluation of Physical Stability of the U-ZrH System," U.P.B. Sci. Bull., Series B, Vol. 75, No. 1, 2013.

2. Beck, R., 1962, “Zirconium-Hydrogen Phase System," Trans. Am. Soc. Met., Vol. 55, 1962, pp. 555.

3. Keiser, Jr., D. D., D. L. Porter, 2009, High Temperature Liquefaction at the Fuel/Cladding Interface in Zr-Containing Metallic Nuclear Fuels Clad with Iron- and Nickel-based Alloys, INL/EXT-09-15598, August 2009. 
4. Terrani, K. A., M. Balooch, D. Wongsawaeng, S. Jaiyen, and D. R. Olander, 2010, "The kinetics of hydrogen desorption from and adsorption on zirconium hydride," Journal of Nuclear Materials, Vol. 397, 2010, pp. 61-68.

5. Wongsawaeng, D., and S. Jaiyen, 2010, "High-temperature absolute hydrogen desorption kinetics of zirconium hydride under clean and oxidized surface conditions," Journal of Nuclear Materials, Vol. 403, 2010, pp. 19-24.

6. Sheldon, R. I., and D. E. Peterson, 1989, “The U-Zr (uranium-zirconium) system, Bulletin of Alloy Phase Diagrams," Journal of Nuclear Materials, Vol. 10, 1989, pp. 165-171, 205-206.

7. La Grange, L. D., L. J. Dykstra, J. M. Dixon, and U. Merten, 1959, “A study of the zirconium-hydrogen and zirconium-hydrogen-uranium systems between 600 and $800^{\circ}$," Journal of Physical Chemistry, Vol. 63, 1959, pp. 2035-2040.

8. Basak, C. B., S. Neogy, D. Srivastava, G. K. Dey, and S. Banerjee, 2011, "Disordered bcc gamma-phase to delta-phase transformation in Zr-rich U-Zr alloy," Philosophical Magazine, Vol. 91, 2011, pp. 3290-3306.

9. NUREG-1282, 1987, "Safety Evaluation Report on High-Uranium Content, Low Enriched Uranium-Zirconium Hydride Fuels for TRIGA Reactors," Docket No. 50-163, U.S. Nuclear Regulatory Commission, August 1987. 